J. BROS

G.A. VIANO

Connection between the algebra of kernels on the sphere and the Volterra algebra on the one-sheeted hyperboloid : holomorphic "perikernels"

Bulletin de la S. M. F., tome 120, no 2 (1992), p. 169-225

<http://www.numdam.org/item?id=BSMF_1992_120_2_169_0>

(C) Bulletin de la S. M. F., 1992, tous droits réservés.

L'accès aux archives de la revue «Bulletin de la S. M. F. » (http: //smf.emath.fr/Publications/Bulletin/Presentation.html) implique l'accord avec les conditions générales d'utilisation (http://www.numdam.org/ conditions). Toute utilisation commerciale ou impression systématique est constitutive d'une infraction pénale. Toute copie ou impression de ce fichier doit contenir la présente mention de copyright.

\title{
Numdam
}

Article numérisé dans le cadre du programme

Numérisation de documents anciens mathématiques

http://www.numdam.org/ 
CONNECTION BETWEEN THE ALGEBRA

OF KERNELS ON THE SPHERE AND THE

VOLTERRA ALGEBRA ON THE ONE-SHEETED

HYPERBOLOID : HOLOMORPHIC "PERIKERNELS"

BY

J. BROS AND G.A. VIANO (*)

\begin{abstract}
RÉSUMÉ. - On établit une relation de type prolongement analytique entre l'algèbre des noyaux sur la sphère $\$_{d-1}$ et l'algèbre des noyaux de Volterra sur l'hyperboloïde à une nappe $X_{d-1}$; cette relation est réalisée au moyen d'une algèbre de fonctions holomorphes (appelées "périnoyaux") sur l'hyperboloïde complexe dans $\mathbb{C}^{d}$; le produit de composition définissant cette algèbre fait intervenir des cycles d'intégration mobiles permettant le passage de $\mathbb{S}_{d-1}$ à $X_{d-1}$ par le domaine complexe.

L'extension de ce résultat au cas d'algèbres de Volterra de noyaux-distributions (incluant l'étude microlocale correspondante) est également effectuée.

ABSTRACT. - The algebra of kernels on the unit sphere $\mathbb{S}_{d-1}$ and the algebra of Volterra kernels on the one-sheeted hyperboloid $X_{d-1}$ are shown to be related (through an analytic continuation procedure involving the distortion of integration cycles) by an algebra of holomorphic functions, called "perikernels" on the complex unit hyperboloid in $\mathbb{C}^{d}$. This result is then extended to the case of Volterra algebras of distribution-kernels.
\end{abstract}

\title{
Introduction
}

The present work has been motivated by analytic structural properties of Quantum Field Theory which concern the passage from the so-called "euclidean framework" of this theory (involving purely imaginary times and energies) to the "minkowskian framework" in real $d$-dimensional relativistic space-time.

(*) Texte reçu le 30 juillet 1990.

J. Bros, Service de Physique Théorique, Direction des Sciences de la Matière, CEASaclay, 91191 Gif-sur-Yvette Cedex, France

G.A. Viano, Istituto Nazionale Fisica Nucleare, Sezione di Genova, Dipartimento di Fisica dell'Universita di Genova, Italy.

BULLETIN DE LA SOCIÉTÉ MATHÉMATIQUE DE FRANCE 0037-9484/1992/169/\$5.00

(C) Société mathématique de France 
More specifically, one of the basic procedures involved in the theory, namely Feynman-type integration, sets the problem of the connection by analytic continuation between the algebra of kernels on the sphere $\mathbb{S}_{d-1}$ and an appropriate algebra of kernels on the one-sheeted hyperboloid $X_{d-1}$. As a matter of fact, since $X_{d-1}$ is equipped with the partial ordering of the ambient minkowskian space $\mathbb{R}^{d}$ (namely $x \geq y \Leftrightarrow x-y$ belongs to the closed future cone of $\mathbb{R}^{d}$ ), the relevance of the algebra of Volterra kernels in the sense of [1] and [2] (i.e. kernels $K(x, y)$ whose support is contained in the set $\left.\left\{(x, y) \in X_{d-1} \times X_{d-1} ; x \geq y\right\}\right)$ emerges in a natural way. Then the idea of analytic continuation from $\mathbb{S}_{d-1}$ to $X_{d-1}$ leads one to regard these sets as submanifolds of the unit complex hyperboloid $X_{d-1}^{(c)}$ in the complex minkowskian space $\mathbb{C}^{d}$, and to introduce holomorphic kernel-type functions on "cut-domains" $\Delta_{\mu}$ of the form $X_{d-1}^{(c)} \times X_{d-1}^{(c)} \backslash \Sigma_{\mu}^{(c)}$, where the "cut" $\Sigma_{\mu}^{(c)}$ is a certain analytic hypersurface.

It is the purpose of this work to define an appropriate $*^{(c)}$-product on this class of functions, called "perikernels" on $X_{d-1}^{(c)}$ in the following, by an integration procedure involving a certain class of cycles in $X_{d-1}^{(c)}$. By using a contour distortion technique, this $*^{(c)}$-product will then be shown to interpolate between :

i) the usual composition product (denoted by $*$ ) of kernels on the sphere $\mathbb{S}_{d-1}$;

ii) the composition product (denoted by $\diamond$ ) of Volterra kernels on $X_{d-1}$.

While i) will be obtained simply by taking the restriction of perikernels to $\mathbb{S}_{d-1} \times \mathbb{S}_{d-1}$, ii) will involve relevant discontinuity functions of the perikernels across the "cut" $\Sigma_{\mu}^{(c)}$.

By adopting the same analytic continuation viewpoint in a forthcoming paper, we shall display the connection (via $X_{d-1}^{(c)}$ ) between the harmonic analysis on the sphere $\mathbb{S}_{d-1}$ and the harmonic analysis on the one-sheeted hyperboloid $X_{d-1}$. To this purpose, we shall consider there a subclass of perikernels satisfying additional invariance properties with respect to the complex Lorentz group acting on $X_{d-1}^{(c)}$. Then, the previous $*^{(c)}$-, *and $\diamond$-products will be turned into "ordinary products" by making use of appropriate Fourier-Laplace-type transformations.

The present paper is organized as follows.

In view of its simplicity, the one-dimensional case (corresponding to the complex hyperbola $X_{1}^{(c)}$ in $\mathbb{C}^{2}$ ) has been presented in section 1 ; it exhibits clearly the connection (by analytic continuation) between periodic kernels

TOME $120-1992-\mathrm{N}^{\circ} 2$ 
and Volterra kernels on the real line. Definitions and simple geometrical facts concerning the general case (i.e. perikernels on $X_{d-1}^{(c)}$, for all $d \geq 3$ ) are given in section 2 ; section 3 is then devoted to the proof of our main result concerning the $*^{(c)}$-product and the connection that it provides between the $*$ and $\diamond$-products, respectively on $\mathbb{S}_{d-1}$ and $X_{d-1}$.

At the end of section 3 , we also consider the case when the holomorphic perikernels have a slowly increasing behaviour near the cut $\Sigma_{\mu}^{(c)}$, which ensures the existence of boundary values in the sense of distributions. This analysis then leads to the consideration of Volterra algebras on $X_{d-1}$ whose elements are distribution kernels. For completeness, a microlocal study in the framework of singular spectrum theory [3], [4], [5], [6] is also presented.

In the last part of this paper (i.e. section 4), we shall consider a slightly more general case where the domain $\Delta_{\mu}$ in $X_{d-1}^{(c)} \times X_{d-1}^{(c)}$ is bordered by two "cuts" $\Sigma_{\mu}^{(c)}$ and $\Sigma_{\mu}^{\prime}(c)$, whose relative position corresponds to a certain symmetry of the complex hyperboloid $X_{d-1}^{(c)}$ which we shall call "crossing symmetry" . The relevance of perikernels holomorphic in such symmetric domains $\Delta_{\mu}$ is again suggested by the structural properties of Quantum Field Theory, in which the notion of "crossing symmetry" has a deep physical meaning. In section 4 , we extend the result of section 3 concerning the $*^{(c)}$-product of perikernels to this more general geometrical situation.

\section{The one-dimensional case}

\subsection{Convolution of periodic functions and Volterra-convolu- tion on $\mathbb{R}^{+}$: their connection by analytic continuation.}

In the complex plane $\mathbb{C}_{(\theta)}$ of the variable $\theta=u+i v$, we consider for each $\mu>0$ the cut-domain $\mathcal{J}_{+}^{(\mu)}=\mathcal{J}_{+} \backslash \Xi(\mu)$, where

$$
\begin{aligned}
\Xi(\mu) & =\{\theta \in \mathbb{C} ; \theta=2 k \pi+i v, v \geq \mu, k \in \mathbb{Z}\} \\
\mathcal{J}_{+} & =\{\theta \in \mathbb{C} ; \operatorname{Im} \theta>0\} .
\end{aligned}
$$

We put $\dot{\mathcal{J}}_{+}=\mathcal{J}_{+} / 2 \pi \mathbb{Z}, \dot{\mathcal{J}}_{+}^{(\mu)}=\mathcal{J}_{+}^{(\mu)} / 2 \pi \mathbb{Z}$, and we denote by $O^{(0)}\left(\dot{\mathcal{J}}_{+}^{(\mu)}\right)$ the space of functions $f(\theta)$ satisfying the properties :

i) $\forall k \in \mathbb{Z}, f(\theta)=f(\theta+2 k \pi)$;

ii) $f$ is holomorphic in $\mathcal{J}_{+}^{(\mu)}$ and admits a continuous boundary value on $\mathbb{R}$, denoted by $\mathbf{f}(u)$; 
iii) the limits $f_{\varepsilon}(v)=\lim _{\substack{u \rightarrow 0 \\ u>0}} f(\varepsilon u+i v), \varepsilon=+$ or - , exist in $\mathcal{C}^{(0)}\left(\mathbb{R}^{+}\right)$, and one puts

$$
\mathrm{f}(v)=\Delta f(v) \equiv i\left[f_{+}(v)-f_{-}(v)\right]
$$

f belongs to the space $\mathcal{C}_{(\mu)}^{(0)}\left(\mathbb{R}^{+}\right)$of continuous functions on $\mathbb{R}^{+}$whose support in contained in $[\mu,+\infty[$.

We denote by $*$ the convolution product on $\mathbb{R} / 2 \pi \mathbb{Z}$, namely : for every $g_{1}$ and $g_{2}$ in $\mathcal{C}^{(0)}(\mathbb{R} / 2 \pi \mathbb{Z})$,

$$
g(u)=\left(g_{1} * g_{2}\right)(u)=\int_{0}^{2 \pi} g_{1}\left(u-u^{\prime}\right) g_{2}\left(u^{\prime}\right) \mathrm{d} u^{\prime}
$$

and $g \in \mathcal{C}^{(0)}(\mathbb{R} / 2 \pi \mathbb{Z})$.

On the other hand, we denote by $\diamond$ the "Volterra convolution product" on $\mathbb{R}^{+}$defined as follows : for every couple $\left(\mathrm{f}_{1}, \mathrm{f}_{2}\right)$ with $\mathrm{f}_{i} \in \mathcal{C}_{\left(\mu_{i}\right)}^{(0)}\left(\mathbb{R}^{+}\right)$, $i=1,2$,

$$
\mathrm{f}(v)=\left(\mathrm{f}_{1} \diamond \mathrm{f}_{2}\right)(v)=\int_{0}^{v} \mathrm{f}_{1}\left(v-v^{\prime}\right) \mathrm{f}_{2}\left(v^{\prime}\right) \mathrm{d} v^{\prime}
$$

$\mathrm{f}(v)$ belongs to the space $\mathcal{C}_{\left(\mu_{1}+\mu_{2}\right)}^{(0)}\left(\mathbb{R}^{+}\right)$.

The connection between these two convolution products is provided by the following property.

Proposition 1. - For every couple $\left(f_{1}, f_{2}\right)$, with $f_{i} \in O^{(0)}\left(\dot{\mathcal{J}}_{+}^{\left(\mu_{i}\right)}\right)$, $i=1,2$, there exists a unique function $f$ in $O^{(0)}\left(\dot{\mathcal{J}}_{+}^{\left(\mu_{1}+\mu_{2}\right)}\right)$, which we denote by $f=f_{1} *^{(c)} f_{2}$, such that:

i) $\mathbf{f}=\mathbf{f}_{1} * \mathbf{f}_{2}$;

ii) $\Delta f=\Delta f_{1} \diamond \Delta f_{2}$;

iii) for every $\theta=u+i v$ in $\mathcal{J}_{+}^{\left(\mu_{1}+\mu_{2}\right)}$, there exists a class of cycles $\dot{\gamma}(\theta)$ in $H^{1}\left(\dot{D}_{\mu}^{\theta}\right)$, where $\dot{D}_{\mu}^{\theta}=D_{\mu}^{\theta} / 2 \pi \mathbb{Z}$, and

$$
D_{\mu}^{\theta}=\left\{\theta^{\prime} \in \mathbb{C} ; \theta^{\prime} \in \mathcal{J}_{+}^{\left(\mu_{2}\right)}, \theta-\theta^{\prime} \in \mathcal{J}_{+}^{\left(\mu_{1}\right)}\right\}
$$

such that:

$$
f(\theta)=\int_{\dot{\gamma}(\theta)} f_{1}\left(\theta-\theta^{\prime}\right) f_{2}\left(\theta^{\prime}\right) \mathrm{d} \theta^{\prime}
$$

томе $120-1992-\mathrm{N}^{\circ} 2$ 
Proof. - For every $\theta$ in $\dot{\mathcal{J}}_{+}^{\left(\mu_{1}+\mu_{2}\right)}$ (represented e.g. by the strip $\{\theta ;-\pi \leq \operatorname{Re} \theta \leq \pi\}$ ) we define the class $\dot{\gamma}(\theta)$ of iii) by a representative $\gamma(\theta)$ of the form illustrated by Figure 1 . Since $\dot{D}_{\mu}^{\theta}$ can be identified with (e.g.) the "cut-rectangle"

$$
\begin{aligned}
& \left\{\theta^{\prime}=u^{\prime}+i v^{\prime} ;-\pi \leq u^{\prime} \leq \pi, 0<v^{\prime}<v,\right. \\
& \left.\theta^{\prime} \notin \Xi\left(\mu_{2}\right), \theta-\theta^{\prime} \notin \Xi\left(\mu_{1}\right)\right\},
\end{aligned}
$$

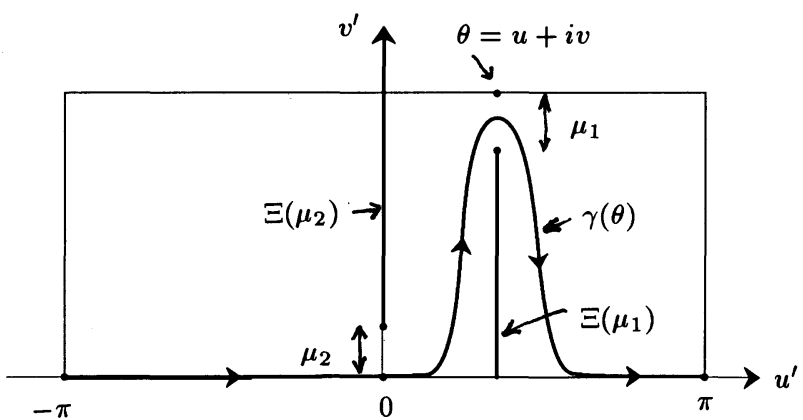

Figure 1.

it is clear that $\gamma(\theta)$ represents the unique generator of $H^{1}\left(\dot{D}_{\mu}^{\theta}\right)$. The assumptions on $f_{1}$ and $f_{2}$ imply that the integrand of $(3)$ is an analytic function of $\left(\theta, \theta^{\prime}\right)$ in $\bigcup_{\theta \in \dot{J}_{+}^{\left(\mu_{1}+\mu_{2}\right)}}\left(\{\theta\} \times \dot{D}_{\mu}^{\theta}\right)$ which is a (locally trivial) fiber bundle with basis $\dot{J}_{+}^{\left(\mu_{1}+\mu_{2}\right)}$; then there exists correspondingly (see e.g. [7], [8]) a homology bundle with fibers $H^{1}\left(\dot{D}_{\mu}^{\theta}\right)$ over $\dot{\mathcal{J}}_{+}^{\left(\mu_{1}+\mu_{2}\right)}$, and since the set $\bigcup_{\theta \in \dot{J}_{+}^{\left(\mu_{1}+\mu_{2}\right)}}(\{\theta\} \times \dot{\gamma}(\theta))$ is a continuous section of the latter (described explicitly by a "continuous distortion" of the cycle $\gamma(\theta)$ of Figure 1, for $\theta$ varying in $\dot{\mathcal{J}}_{+}^{\left(\mu_{1}+\mu_{2}\right)}$ ), the integral (3) defines $f(\theta)$ as an analytic function in $\dot{\mathcal{J}}_{+}^{\left(\mu_{1}+\mu_{2}\right)}$. The continuity assumptions on $f_{1}, f_{2}$ allow one to take limiting representatives of $\dot{\gamma}(\theta)$ in $\dot{\bar{D}_{\mu}^{\theta}}$, and in particular to choose $\gamma(\theta)=[-\pi, \pi]$ for $0 \leq v=\operatorname{Im} \theta<\mu_{2}$ : the boundary value of $f$ is therefore defined as a continuous function on $\mathbb{R} / 2 \pi \mathbb{Z}$ given by the $*$-convolution formula of i). On the other hand, when $\theta$ tends to the set $\Xi\left(\mu_{1}+\mu_{2}\right)$ from the respective sides $\varepsilon \operatorname{Re} \theta>0, \varepsilon=+$ or - , the situation of $\gamma(\theta)$ is described correspondingly in Figure 2.a and Figure 2.b; in the limit, one obtains the functions $f_{\varepsilon}(v)=\lim _{\substack{u \rightarrow 0 \\ u>0}} f(\varepsilon u+i v)$ via the following formulae (deduced from (3)) : 


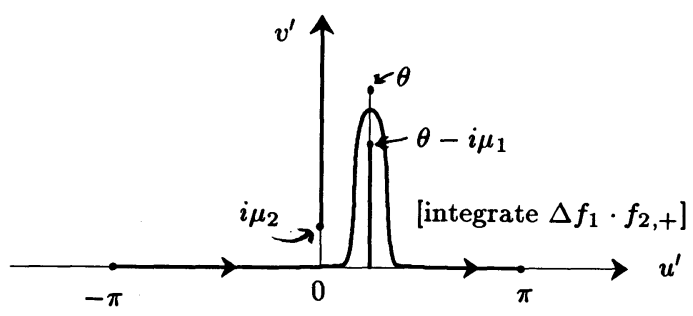

Figure 2.a.

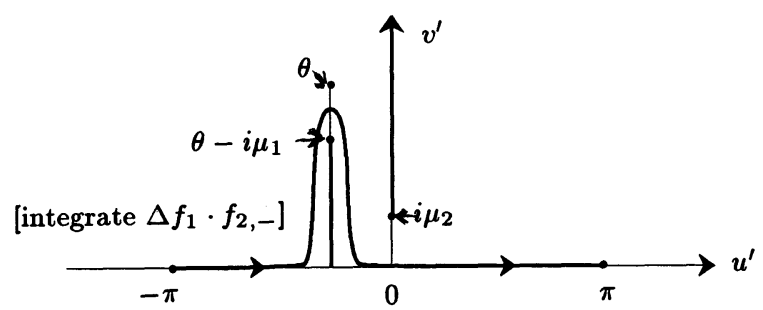

Figure 2.b.

(4) $f_{\varepsilon}(v)=\int_{[-\pi, 0[U] 0, \pi]} f_{1}\left(i v-\theta^{\prime}\right) f_{2}\left(\theta^{\prime}\right) \mathrm{d} \theta^{\prime}$

$$
+\int_{\mu_{1}}^{v-\mu_{2}} \Delta f_{1}\left(v-v^{\prime}\right) f_{2, \varepsilon}\left(i v^{\prime}\right) \mathrm{d} v^{\prime} .
$$

It then follows that

$$
\Delta f(v)=i\left[f_{+}(v)-f_{-}(v)\right]=\int_{\mu_{1}}^{v-\mu_{2}} \Delta f_{1}\left(v-v^{\prime}\right) \cdot \Delta f_{2}\left(v^{\prime}\right) \mathrm{d} v^{\prime}
$$

which expresses property ii)

The case of distribution boundary values

For any positive integer $s$, we denote by $O_{s}\left(\dot{J}_{+}^{(\mu)}\right)$ the space of functions $f(\theta)$, analytic in $\dot{J}^{(\mu)}$, which are of the form $f=\mathrm{d}^{s} g / \mathrm{d} \theta^{s}$, with $g$ in $O^{(0)}\left(\dot{\mathcal{J}}_{+}^{(\mu)}\right)$. From the theory of distributions as boundary values of holomorphic functions [9], we know that if $f \in O_{s}\left(\dot{\mathcal{J}}_{+}^{(\mu)}\right), f(u+i v)$ is "of slow

$$
\text { TOME } 120-1992-\mathrm{N}^{\circ} 2
$$


increase" near the cut $\Xi(\mu)=\left[i \mu,+\infty\left[\right.\right.$ (i.e. is bounded by $M(v)|u|^{-s}$ ) and admits boundary values $f_{+}, f_{-}$on $\Xi(\mu)$ in the sense of distributions; $f_{+}, f_{-}$and $\mathrm{f}=\Delta f=i\left(f_{+}-f_{-}\right)$are distributions of bounded order $(\leq s-1)$ on $\mathbb{R}_{(v)}$, with support contained in $\Xi(\mu)$, and all such distributions $\mathrm{f}$ can be generated in this way. Similarly each function $f$ in $O_{s}\left(\dot{\mathcal{J}}_{+}^{(\mu)}\right)$

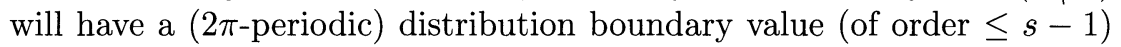
on $\mathbb{R}$.

The convolution product $f=f_{1} *^{(c)} f_{2}$ of two functions $f_{i}$ in $O_{s_{i}}\left(\dot{\mathcal{J}}_{+}^{\left(\mu_{i}\right)}\right)$ $(i=1,2)$ can still be defined by the integral formula (3) and it satisfies the properties of Proposition 2, the Volterra convolution product $\Delta f=\Delta f_{1} \diamond \Delta f_{2}$ being still defined by formula (2) in the sense of distributions. In fact, this follows from the representation $f_{i}=\mathrm{d}^{s_{i}} g_{i} / \mathrm{d} \theta^{s_{i}}$, for functions in $O_{s_{i}}\left(\dot{\mathcal{J}}_{+}^{\left(\mu_{i}\right)}\right)$ and from the relations :

$$
\left(g_{1} *^{(c)} g_{2}\right)^{\left(s_{1}+s_{2}\right)}=g_{1}^{\left(s_{1}\right)} *^{(c)} g_{2}^{\left(s_{2}\right)}
$$

(checked for $\theta$ in the holomorphy domain $\dot{\mathcal{J}}_{+}^{\left(\mu_{1}+\mu_{2}\right)}$ ) and

$$
\begin{aligned}
& \left(\mathbf{g}_{1} * \mathbf{g}_{2}\right)^{\left(s_{1}+s_{2}\right)}=\mathbf{g}_{1}^{\left(s_{1}\right)} * \mathbf{g}_{2}^{\left(s_{2}\right)}, \\
& \left(g_{1} \diamond g_{2}\right)^{\left(s_{1}+s_{2}\right)}=g_{1}^{\left(s_{1}\right)} \diamond g_{2}^{\left(s_{2}\right)} .
\end{aligned}
$$

respectively on $\mathbb{S}_{1}$ and $\mathbb{R}^{+}$; the latter being valid in the sense of distributions.

\subsection{Kernels on $\mathbb{S}_{1}$, Volterra kernels on $\mathbb{R}^{+}$and associated perikernels.}

In this subsection, we consider algebras of kernels instead of the convolution algebras of functions considered in $\S 1.1$, and we shall establish a connection by analytic continuation (similar to that of Proposition 1) between the algebra of $2 \pi$-periodic kernels $\underline{\mathbf{K}}\left(u, u^{\prime}\right)$ on $\mathbb{R}$ (or kernels on $\mathbb{S}_{1}$ ) $\left(\forall\left(k, k^{\prime}\right) \in \mathbb{Z} \times \mathbb{Z}, \underline{\mathbf{K}}\left(u+2 k \pi, u^{\prime}+2 k^{\prime} \pi\right)=\underline{\mathbf{K}}\left(u, u^{\prime}\right)\right)$ and the algebra of "Volterra kernels" on $\mathbb{R}^{+}$; by "Volterra kernel", we mean a kernel $\underline{K}\left(v, v^{\prime}\right)$ whose support is contained in the set $\left\{\left(v, v^{\prime}\right) \in \mathbb{R}^{+} \times \mathbb{R}^{+} ; v \geq v^{\prime}\right\}$.

These two algebras are respectively defined by means of the composition products :

$$
\begin{aligned}
& \left(\underline{\mathbf{K}}_{1} * \underline{\mathbf{K}}_{2}\right)\left(u, u^{\prime}\right)=\int_{0}^{2 \pi} \underline{\mathbf{K}}_{1}\left(u, u^{\prime \prime}\right) \underline{\mathbf{K}}_{2}\left(u^{\prime \prime}, u^{\prime}\right) \mathrm{d} u^{\prime \prime}, \\
& \left(\underline{K}_{1} \diamond \underline{K}_{2}\right)\left(v, v^{\prime}\right)=\int_{v^{\prime}}^{v} \underline{K}_{1}\left(v, v^{\prime \prime}\right) \underline{K}_{2}\left(v^{\prime \prime}, v^{\prime}\right) \mathrm{d} v^{\prime \prime} .
\end{aligned}
$$


To perform this connection, we introduce the space $\mathcal{P}_{\mu}$ of functions $\underline{\mathcal{K}}\left(\theta, \theta^{\prime}\right)$, with $\theta=u+i v, \theta^{\prime}=u^{\prime}+i v^{\prime}$, called "perikernels", which satisfy the following properties :

i) $\forall\left(k, k^{\prime}\right) \in \mathbb{Z} \times \mathbb{Z}, \quad \underline{\mathcal{K}}\left(\theta+2 k \pi, \theta^{\prime}+2 k^{\prime} \pi\right)=\underline{\mathcal{K}}\left(\theta, \theta^{\prime}\right)$;

ii) $\underline{\mathcal{K}}$ is holomorphic in the ("cut-tube") domain

$$
\mathcal{T}_{+}^{(\mu)}=\left(\mathcal{J}_{+} \times \mathcal{J}_{+}\right) \backslash \sigma(\mu)
$$

where $\sigma(\mu)=\left\{\left(\theta, \theta^{\prime}\right) \in \mathbb{C}^{2} ; \theta-\theta^{\prime}=2 k \pi+i \rho, k \in \mathbb{Z}, \rho \geq \mu\right\}$;

iii) $\underline{\mathcal{K}}$ is defined and continuous in the set $\left(\overline{\mathcal{J}}_{+} \times \overline{\mathcal{J}}_{+}\right) \backslash \sigma(\mu)$, and its boundary value on $\mathbb{R} \times \mathbb{R}$ is denoted by $\underline{\mathbf{K}}\left(u, u^{\prime}\right)$;

iv) $\underline{\mathcal{K}}$ is continuous on the set $\sigma(\mu) \cap\left(\mathcal{J}_{+} \times \mathcal{J}_{+}\right)$from both sides and one puts for $\varepsilon=+$ or $-, \forall v, v^{\prime},\left(v>0, v^{\prime}>0\right)$,

$$
\begin{aligned}
& \underline{\mathcal{K}}_{\varepsilon}\left(v, v^{\prime}\right)=\lim _{\substack{u \rightarrow 0 \\
u>0}} \underline{\mathcal{K}}\left(\varepsilon u+i v, i v^{\prime}\right) ; \\
& \underline{K}\left(v, v^{\prime}\right)=\Delta \underline{\mathcal{K}}\left(v, v^{\prime}\right) \equiv i\left[\underline{\mathcal{K}}_{+}\left(v, v^{\prime}\right)-\underline{\mathcal{K}}_{-}\left(v, v^{\prime}\right)\right] .
\end{aligned}
$$

It follows from ii) that $\underline{K}$ belongs to the space $\mathcal{A}_{\mu}$ of continuous functions on $\mathbb{R}^{+} \times \mathbb{R}^{+}$whose support is contained in the set $\left\{\left(v, v^{\prime}\right) ; v-v^{\prime} \geq \mu\right\}$, and which enjoy the following analyticity property : $\forall v$, with $v \geq \mu$, $\underline{K}(v+\lambda, \lambda)$ admits an analytic continuation with respect to $\lambda\left(\right.$ from $\mathbb{R}^{+}$) in the half-plane $\operatorname{Re} \lambda>0 . \mathcal{A}_{\mu}$ is a subspace of the algebra of Volterra kernels on $\mathbb{R}_{+}$, and the following statement is readily obtained :

Proposition 2.

If $\underline{K}_{1} \in \mathcal{A}_{\mu_{1}}$ and $\underline{K}_{2} \in \mathcal{A}_{\mu_{2}}$, then $\underline{K}_{1} \diamond \underline{K}_{2} \in \mathcal{A}_{\mu_{1}+\mu_{2}}$.

On the other hand, the boundary value $\underline{\mathbf{K}}$ of $\underline{\mathcal{K}}$ introduced in iii) belongs to the algebra of kernels on $\mathbb{S}_{1}$.

By using an argument completely similar to the proof of PROPOSITION 1, we shall introduce a structure of algebra on the space of all perikernels $\mathcal{P}=\lim \mathcal{P}_{\mu}(\mu>0)$, by means of an appropriate composition product $*^{(c)}$, consistent with the $\diamond$ - and $*$-products considered above namely, we will show :

Proposition 3. - For every couple of perikernels $\left(\underline{\mathcal{K}}_{1}, \underline{\mathcal{K}}_{2}\right)$, with $\underline{\mathcal{K}}_{i} \in \mathcal{P}_{\mu_{i}}, i=1,2$, there exists a unique perikernel $\underline{\mathcal{K}}$ in $\underline{\mathcal{P}}_{\mu_{1}+\mu_{2}}$ which we denote by $\underline{\mathcal{K}}=\underline{\mathcal{K}}_{1} *^{(c)} \underline{\mathcal{K}}_{2}$ such that :

i) $\underline{\mathbf{K}}=\underline{\mathbf{K}}_{1} * \underline{\mathbf{K}}_{2}$;

TOME $120-1992-\mathrm{N}^{\circ} 2$ 
ii) $\Delta \underline{\mathcal{K}}=\Delta \underline{\mathcal{K}}_{1} \diamond \Delta \underline{\mathcal{K}}_{2}$;

iii) for every $\left(\theta, \theta^{\prime}\right)$ in $\mathcal{T}_{+}^{\left(\mu_{1}+\mu_{2}\right)}$, the $*^{(c)}$-product is defined by the following integral

$$
\underline{\mathcal{K}}\left(\theta, \theta^{\prime}\right)=\int_{\dot{\gamma}\left(\theta, \theta^{\prime}\right)} \underline{\mathcal{K}}_{1}\left(\theta, \theta^{\prime \prime}\right) \underline{\mathcal{K}}_{2}\left(\theta^{\prime \prime}, \theta^{\prime}\right) \mathrm{d} \theta^{\prime \prime},
$$

with the following specification for the class $\dot{\gamma}\left(\theta, \theta^{\prime}\right)$

a) if $\operatorname{Im}\left(\theta-\theta^{\prime}\right) \leq 0, \dot{\gamma}\left(\theta, \theta^{\prime}\right)$ is represented by $\theta^{\prime}+[-\pi, \pi]$;

b) if $\operatorname{Im}\left(\theta-\theta^{\prime}\right) \geq 0, \dot{\gamma}\left(\theta, \theta^{\prime}\right)$ is represented by $\theta^{\prime}+\gamma\left(\theta-\theta^{\prime}\right)$, where $\gamma\left(\theta-\theta^{\prime}\right)$ is the cycle defined in the proof of Proposition 1.

Proof. - By putting $\Theta=\theta-\theta^{\prime}$ and $\Theta^{\prime \prime}=\theta^{\prime \prime}-\theta^{\prime}$, we can rewrite Eq. (12) as follows :

$$
\begin{aligned}
\underline{\mathcal{K}}\left(\theta, \theta^{\prime}\right) & =\underline{\mathcal{K}}\left(\Theta+\theta^{\prime}, \theta^{\prime}\right) \\
& =\int_{\gamma\left(\theta, \theta^{\prime}\right)-\theta^{\prime}} \underline{\mathcal{K}}_{1}\left(\Theta+\theta^{\prime}, \Theta^{\prime \prime}+\theta^{\prime}\right) \underline{\mathcal{K}}_{2}\left(\Theta^{\prime \prime}+\theta^{\prime}, \theta^{\prime}\right) \mathrm{d} \Theta^{\prime \prime}
\end{aligned}
$$

If $\operatorname{Im}\left(\theta-\theta^{\prime}\right)=\operatorname{Im} \Theta \leq 0$, the choice $\gamma\left(\theta, \theta^{\prime}\right)-\theta^{\prime}=[-\pi, \pi]$ is appropriate since the set $\left\{\left(\theta^{\prime \prime}, \theta^{\prime}\right) ; \operatorname{Im} \theta^{\prime \prime}=\operatorname{Im} \theta^{\prime}>0\right\}$ belongs to the domain $\mathcal{T}_{+}^{\left(\mu_{2}\right)}$ of $\underline{\mathcal{K}}_{2}$ and since the set $\left\{\left(\theta, \theta^{\prime \prime}\right) ; 0<\operatorname{Im} \theta<\operatorname{Im} \theta^{\prime \prime}\right\}$ belongs to the domain $\mathcal{T}_{+}^{\left(\mu_{1}\right)}$ of $\underline{\mathcal{K}}_{1}$.

If $\operatorname{Im}\left(\theta-\theta^{\prime}\right)=\operatorname{Im} \Theta \geq 0$ and $\left(\theta, \theta^{\prime}\right) \notin \sigma\left(\mu_{1}+\mu_{2}\right)$, the choice $\gamma\left(\theta, \theta^{\prime}\right)-\theta^{\prime}=\gamma\left(\theta-\theta^{\prime}\right)$ given in the proof of Proposition 1 is relevant, since the domain $\left\{\Theta^{\prime \prime} \in D_{\mu}^{(\Theta)}\right\}$ used in the latter is embedded (as easily checked) in the analyticity domain of the integrand $\underline{\mathcal{K}}_{1} \cdot \underline{\mathcal{K}}_{2}$ at the r.h.s. of Eq. (13). Formula (13) therefore defines $\underline{\mathcal{K}}\left(\theta, \theta^{\prime}\right)$ as an analytic function in the domain $\mathcal{T}_{+}^{\left(\mu_{1}+\mu_{2}\right)}$, which proves iii). Property i) follows immediately and property ii) is obtained by the same argument as the similar statement ii) of Proposition 1 (in the variables $\Theta, \Theta^{\prime \prime}$, for each fixed $\left.\theta^{\prime}=i v^{\prime}\right)$.

Remark. - In Proposition 3 the statement $\underline{\mathcal{K}} \in \mathcal{P}_{\mu_{1}+\mu_{2}}$ together with the discontinuity formula of ii) are consistent with the result of Proposition 2.

The class of perikernels $\widetilde{\mathcal{P}}_{\mu}$

Results similar to those of Proposition 3 hold for the set $\widetilde{\mathcal{P}}_{\mu}$ of functions $\underline{\mathcal{K}}\left(\theta, \theta^{\prime}\right)$, satisfying the periodicity condition i) and which are analytic in the domain $\widetilde{\mathcal{T}}_{+}^{(\mu)}=\mathbb{C}^{2} \backslash \sigma(\mu)$ and have continuous boundary 
values (from both sides) on the set $\sigma(\mu)$. The discontinuity formula (11) (now valid for all $\left(v, v^{\prime}\right)$ in $\mathbb{R}^{2}$ ) defines $\underline{K}\left(v, v^{\prime}\right)$ as a Volterra kernel on $\mathbb{R}$ (with support in $\left\{\left(v, v^{\prime}\right) ; v-v^{\prime} \geq \mu\right\}$ ). For this extended class of perikernels $\widetilde{\mathcal{P}}_{\mu}$, the ${ }^{(c)}$-product is still defined through formula (12), but with the additional following specification : if $\left(\theta, \theta^{\prime}\right) \notin \mathcal{T}_{+}^{(\mu)}$, there always exists a complex number $\lambda$ such that $\left(\Theta, \Theta^{\prime}\right)=\left(\theta+\lambda, \theta^{\prime}+\lambda\right) \in \mathcal{T}_{+}^{(\mu)}$ and one then puts :

$$
\begin{aligned}
\underline{\mathcal{K}}\left(\theta, \theta^{\prime}\right) & =\underline{\mathcal{K}}\left(\Theta-\lambda, \Theta^{\prime}-\lambda\right) \\
& =\int_{\dot{\gamma}\left(\Theta, \Theta^{\prime}\right)} \underline{\mathcal{K}}_{1}\left(\Theta-\lambda, \Theta^{\prime \prime}-\lambda\right) \underline{\mathcal{K}}_{2}\left(\Theta^{\prime \prime}-\lambda, \Theta^{\prime}-\lambda\right) \mathrm{d} \Theta^{\prime \prime},
\end{aligned}
$$

$\dot{\gamma}\left(\Theta, \Theta^{\prime}\right)$ being then defined in the situation of Proposition 3 (the value of the integral in (14) being in fact independent of $\lambda$, as it can be seen by contour distortion).

The case of distribution boundary values

As in $\S 1.1$, we can introduce the spaces $\mathcal{P}_{\mu, \mathbf{s}}$ and $\widetilde{\mathcal{P}}_{\mu, \mathbf{s}}$ of perikernels $\underline{\mathcal{K}}\left(\theta, \theta^{\prime}\right)$ which are of the form $\underline{\mathcal{K}}=\mathrm{d}^{\left(s+s^{\prime}\right)} \mathcal{L} / \mathrm{d} \theta^{s} \mathrm{~d} \theta^{\prime s^{\prime}}$, with $\mathcal{L}$ in $\mathcal{P}_{\mu}$ or in $\widetilde{\mathcal{P}}_{\mu}$ and $\mathbf{s}=\left(s, s^{\prime}\right) \in \mathbb{N} \times \mathbb{N}$; each of these kernels admits boundary values $\underline{\mathcal{K}}_{ \pm}\left(v, v^{\prime}\right)$ and an associated Volterra kernel $\underline{K}=\Delta \underline{\mathcal{K}}$ on $\mathbb{R}^{+}$, which are defined as distributions (of bounded order) on $\mathbb{R}^{+} \times \mathbb{R}^{+}$. Similarly, the kernels $\underline{\mathcal{K}}$ in $\mathcal{P}_{\mu, \mathbf{s}}$ have distribution boundary values on $\mathbb{R} \times \mathbb{R}$ (and in fact on $\mathbb{S}_{1} \times \mathbb{S}_{1}$ in view of periodicity conditions) from the tube $\mathcal{J}_{+} \times \mathcal{J}_{+}$. The composition product $\underline{\mathcal{K}}_{1} *^{(c)} \underline{\mathcal{K}}_{2}$ of two kernels $\underline{\mathcal{K}}_{i}(i=1,2)$ in $\mathcal{P}_{\mu, \mathbf{s}_{i}}$ (resp. $\widetilde{\mathcal{P}}_{\mu, \mathbf{s}_{i}}$ ) can still be defined by the integral formula (12) (resp. (12) and (14)) in the complex domain $\mathcal{T}_{+}^{\left(\mu_{1}+\mu_{2}\right)}$ (resp. $\widetilde{\mathcal{T}}_{+}^{\left(\mu_{1}+\mu_{2}\right)}$ ). Moreover, this composition product still satisfies the properties listed in PROPOSITION 3 (as it follows from the representation of $\underline{\mathcal{K}}_{i}$ in terms of derivatives of kernels in $\mathcal{P}_{\mu_{i}}$ or $\widetilde{\mathcal{P}}_{\mu_{i}}$ and from formulae similar to Eqs. (6), (7)), the Volterra $\diamond$-product (and possibly the $*$-product) being now understood in the sense of distributions (i.e. defining an algebra of distribution kernels).

\subsection{The geometrical interpretation : algebra of holomorphic perikernels on the complex hyperbola $X_{1}^{(c)}$.}

We introduce the complex hyperbola (isomorphic to the complex unit circle $\left.\mathbb{S}_{1}^{(c)}\right)$ :

$$
\begin{aligned}
X_{1}^{(c)}=\left\{z=\left(z^{(0)}, z^{(1)}\right)=z(\theta)\right. & \in \mathbb{C}^{2} ; \\
z^{(0)} & \left.=-i \sin \theta, z^{(1)}=\cos \theta, \theta \in \mathbb{C}\right\}
\end{aligned}
$$

TOME $120-1992-\mathrm{N}^{\circ} 2$ 
and two real submanifolds $X_{1}$ and $S_{1}$ of $X_{1}^{(c)}$. The hyperbola

$$
X_{1}=\left\{z \in \mathbb{R}^{2} ; z^{(0)}=\sinh v, z^{(1)}= \pm \cosh v ; v \in \mathbb{R}\right\}
$$

is obtained for $\theta=i v+k \pi, k \in \mathbb{Z}$ and the circle $S_{1}$ in $(i \mathbb{R}) \times \mathbb{R}$ is defined by putting $\theta=u \in \mathbb{R}$ in the equations of $X_{1}^{(c)}$; we also put $X_{1}^{+}=\left\{z=z(i v) \in X_{1} ; v \geq 0\right\}$. On $X_{1}^{(c)}$, we introduce the integration measure $\mathrm{d} \sigma^{(c)}(z)=\mathrm{d} \theta$, and put $\mathrm{d} \sigma_{\mid S_{1}}^{(c)}=\mathrm{d} \sigma(z)=\mathrm{d} u$ and $\left.\mathrm{d} \sigma\right|_{X_{1}} ^{(c)}=i \mathrm{~d} \sigma(z)=i \mathrm{~d} v$.

Let us then consider :

i) the class of kernels $\mathbf{K}\left(z, z^{\prime}\right)$ on $S_{1}$, represented by kernels $\underline{\mathbf{K}}\left(u, u^{\prime}\right)=$ $\mathbf{K}\left(z, z^{\prime}\right) \mid z=z(u), z^{\prime}=z\left(u^{\prime}\right)$, equipped with the composition product :

$$
\left(\mathbf{K}_{1} * \mathbf{K}_{2}\right)\left(z, z^{\prime}\right)=\int_{S} \mathbf{K}_{1}\left(z, z^{\prime \prime}\right) \mathbf{K}_{2}\left(z^{\prime \prime}, z^{\prime}\right) \mathrm{d} \boldsymbol{\sigma}=\left(\underline{\mathbf{K}}_{1} * \underline{\mathbf{K}}_{2}\right)\left(u, u^{\prime}\right) ;
$$

ii) the class of "Volterra kernels" $K\left(z, z^{\prime}\right)$ on $X_{1}^{+}$, represented by Volterra kernels $\underline{K}\left(v, v^{\prime}\right)=\left.K\left(z, z^{\prime}\right)\right|_{z=z(i v), z^{\prime}=z\left(i v^{\prime}\right)}$ on $\mathbb{R}^{+}$, equipped with the composition product

$$
\begin{aligned}
\left(K_{1} \diamond K_{2}\right)\left(z, z^{\prime}\right) & =\int_{z^{\prime \prime} \in X ; z \geq z^{\prime \prime} \geq z^{\prime}} K_{1}\left(z, z^{\prime \prime}\right) K_{2}\left(z^{\prime \prime}, z^{\prime}\right) \mathrm{d} \sigma\left(z^{\prime \prime}\right) \\
& =\left(\underline{K}_{1} \diamond \underline{K}_{2}\right)\left(v, v^{\prime}\right) .
\end{aligned}
$$

In the latter, the relation $z \geq z^{\prime \prime} \geq z^{\prime}$ (corresponding to $v \geq v^{\prime \prime} \geq v^{\prime}$ ) can be given an intrinsic meaning in the ambient space $\mathbb{R}_{(z)}^{2}$ by introducing the cone $\Omega_{+}=\left\{z=\left(z^{(0)}, z^{(1)}\right) \in \mathbb{R}^{2} ; z^{(0)} \geq\left|z^{(1)}\right|\right\}$ and by putting $z \geq z^{\prime}$ if $z-z^{\prime} \in \Omega_{+}$(see in [1], [2] and below in section $2, \S 2.2$, the generalization of the $\diamond$-product of "Volterra-kernels" in dimension $d$ ).

By using the results of $\S 1.2$, we can then introduce algebras of "perikernels" $\mathcal{K}\left(z, z^{\prime}\right)$ on the complex hyperbola $X_{1}^{(c)}$ which will provide an analytic interpolation between appropriate classes of kernels $\mathbf{K}$ on $S_{1}$ and Volterra kernels $K$ on $X_{1}^{+}$, according to the properties described in Proposition 3. We define $\mathcal{P}_{\mu}\left(X_{1}^{(c)}\right)\left(\right.$ resp. $\left.\mathcal{P}_{\mu, \mathbf{s}}\left(X_{1}^{(c)}\right)\right)$ as the set of functions $\mathcal{K}\left(z, z^{\prime}\right)$ holomorphic in the complex manifold

$$
\Delta_{\mu}^{+}=\left\{\left(z, z^{\prime}\right) \in X_{1}^{(c)} \times X_{1}^{(c)} ; z=z(\theta), z^{\prime}=z\left(\theta^{\prime}\right),\left(\theta, \theta^{\prime}\right) \in \mathcal{T}_{+}^{(\mu)}\right\}
$$

represented in $\left(\theta, \theta^{\prime}\right)$-space by a perikernel $\underline{\mathcal{K}}\left(\theta, \theta^{\prime}\right)=\mathcal{K}\left(z(\theta), z\left(\theta^{\prime}\right)\right)$ belonging to $\mathcal{P}_{\mu}\left(\right.$ resp. $\left.\mathcal{P}_{\mu, \mathbf{s}}\right)$. 
For all perikernels $\mathcal{K}$ in these classes, a composition product $\mathcal{K}=$ $\mathcal{K}_{1} *^{(c)} \mathcal{K}_{2}$ is defined by the formula :

$$
\begin{aligned}
\mathcal{K}\left(z, z^{\prime}\right) & =\int_{\Gamma\left(z, z^{\prime}\right)} \mathcal{K}_{1}\left(z, z^{\prime \prime}\right) \mathcal{K}_{2}\left(z^{\prime \prime}, z^{\prime}\right) \mathrm{d} \sigma^{(c)}\left(z^{\prime \prime}\right) \\
& =\left(\underline{\mathcal{K}}_{1} *^{(c)} \underline{\mathcal{K}}_{2}\right)\left(\theta, \theta^{\prime}\right)
\end{aligned}
$$

In the latter, the cycle $\Gamma\left(z, z^{\prime}\right)$ is such that $\left.\Gamma\left(z, z^{\prime}\right)\right|_{z=z(\theta), z^{\prime}=z\left(\theta^{\prime}\right)}=$ $\gamma\left(\theta, \theta^{\prime}\right)$.

Then, in view of Proposition 3 and of Eqs. (15), (16) and (18), the restrictions (or boundary values) $\mathbf{K}, \mathbf{K}_{1}, \mathbf{K}_{2}$ of $\mathcal{K}, \mathcal{K}_{1}, \mathcal{K}_{2}$ to $S_{1} \times S_{1}$ and the corresponding discontinuity functions on $X_{1}^{+} \times X_{1}^{+}$, namely the Volterra kernels $\Delta \mathcal{K}, \Delta \mathcal{K}_{1}, \Delta \mathcal{K}_{2}$ on $X_{1}^{+}$(represented respectively by $\Delta \underline{\mathcal{K}}$, $\left.\Delta \underline{\mathcal{K}}_{1}, \Delta \underline{\mathcal{K}}_{2}\right)$, satisfy the relations $\mathbf{K}=\mathbf{K}_{1} * \mathbf{K}_{2}$ and $\Delta \mathcal{K}=\Delta \mathcal{K}_{1} \diamond \Delta \mathcal{K}_{2}$.

Similar classes of perikernels, denoted by $\widetilde{\mathcal{P}}_{\mu}\left(X_{1}^{(c)}\right)$ and $\widetilde{\mathcal{P}}_{\mu, \mathbf{s}}\left(X_{1}^{(c)}\right)$, can be introduced; these perikernels are analytic in a domain $\widetilde{\Delta}_{\mu}^{+}$(larger than $\Delta_{\mu}^{+}$) obtained by replacing $\mathcal{T}_{+}^{(\mu)}$ by $\widetilde{\mathcal{T}}_{+}^{(\mu)}$ in formula $(17) . \widetilde{\Delta}_{\mu}^{+}$can also be described as the full complex manifold $X_{1}^{(c)} \times X_{1}^{(c)}$ deprived from the "cut" :

$$
\begin{aligned}
\Sigma_{\mu}^{(c)+}=\left\{\left(z, z^{\prime}\right) \in X_{1}^{(c)} \times X_{1}^{(c)}\right. & \\
\left(z^{(0)}-z^{\prime(0)}\right)^{2}-\left(z^{(1)}-z^{(1)}\right)^{2} \geq 2(\cosh \mu-1) & \left.\operatorname{det}\left(z, z^{\prime}\right) \geq 0\right\} .
\end{aligned}
$$

The previous results on composition products $\left(*^{(c)}\right.$, * and $\left.\diamond\right)$ hold similarly for these perikernels, the discontinuity functions $\Delta \mathcal{K}, \Delta \mathcal{K}_{1}$, $\Delta \mathcal{K}_{2}$ being now Volterra kernels on the whole branch of hyperbola $Y=X \cap\left\{z ; z^{(1)}>0\right\}$. The action of the connected complex Lorentz group $\mathrm{SO}_{0}(1,1)^{(c)}\left(\approx \mathrm{SO}_{0}(2, \mathbb{C})\right)$ on $X_{1}^{(c)}$ can be defined as follows :

$$
\begin{gathered}
\forall g=g_{\theta} \in \mathrm{SO}_{0}(1,1)^{(c)}, \forall z_{1}=z\left(\theta_{1}\right) \in X_{1}^{(c)}, \\
z_{2}=g_{\theta} z_{1}=z\left(\theta_{2}\right) \text { with } \theta_{2}=\theta+\theta_{1},
\end{gathered}
$$

the corresponding actions of $\mathrm{SO}_{0}(2, \mathbb{R})$ (resp. $\left.\mathrm{SO}_{0}(1,1)\right)$ on $S_{1}\left(\right.$ resp. $X_{1}$ ) being obtained for $\theta$ in $\mathbb{R}$ (resp. in $i \mathbb{R}+k \pi$ ).

We can then consider for each previous class of perikernels $\mathcal{K}$, the subclass containing those perikernels which satisfy the invariance property :

TOME $120-1992-\mathrm{N}^{\circ} 2$ 
$\forall g \in \mathrm{SO}_{0}(1,1)^{(c)}, \mathcal{K}\left(g z, g z^{\prime}\right)=\mathcal{K}\left(z, z^{\prime}\right)$. Such perikernels $\mathcal{K}\left(z, z^{\prime}\right)$ are identified with analytic functions of a single complex variable $f$ by the formula $\mathcal{K}\left(z(\theta), z\left(\theta^{\prime}\right)\right)=f\left(\theta-\theta^{\prime}\right)$. By introducing the point $z_{0}=z(0)=(0,1)$ of $X_{1}$, we can also write : $\mathcal{K}\left(z(\theta), z_{0}\right)=f(\theta)$. Then for these perikernels (in particular for those in $\mathcal{P}_{\mu}$ ), our previous results concerning the $*^{(c)}{ }_{-}$, *- and $\diamond$-products reduce exactly to our Proposition 1 for convolution products on $\dot{\mathcal{J}}_{+}^{(\mu)}$, and are thus interpretable as describing a convolution on the complex group $\mathrm{SO}_{0}(1,1)^{(c)}$.

\section{The general case : geometry of the problem}

\subsection{The manifolds $X^{(c)}, X, S$, and the related groups of matrices.}

For $d \geq 3$, we consider the space $\mathbb{C}^{d}$ of variables $\left(z^{(0)}, z^{(1)}, \ldots, z^{(d-1)}\right)$ $=\left(z^{(0)}, \vec{z}\right)$ equipped with the minkowskian quadratic form :

$$
z^{2}=z^{(0)^{2}}-z^{(1)^{2}}-\cdots-z^{(d-1)^{2}}
$$

and we put $: \vec{z}^{2}=z^{(1)^{2}}+\cdots+z^{(d-1)^{2}}$, i.e. $z^{2}=z^{(0)^{2}}-\vec{z}^{2}$; the minkowskian scalar product of two vectors $z, z^{\prime}$ is denoted by

$$
z \cdot z^{\prime} \equiv z^{(0)} z^{(0)}-z^{(1)} z^{(1)}-\cdots-z^{(d-1)} z^{(d-1)} \equiv z^{(0)} z^{(0)}-\vec{z} \cdot \vec{z}^{\prime} .
$$

The cone $\Omega_{+}=\left\{z \in \mathbb{R}^{d} ; z^{2} \geq 0, z^{(0)} \geq 0\right\}$ ("closed forward light cone") defines on $\mathbb{R}^{d}$ the following partial ordering relation : $z_{1} \geq z_{2}$ (or $z_{2} \leq z_{1}$ ) if $z_{1}-z_{2} \in \Omega_{+}$. We call $\chi$ the symmetry : $z=\left(z^{(0)}, \vec{z}\right) \mapsto \chi(z)=\left(-z^{(0)}, \vec{z}\right)$ whose restriction to $\mathbb{R}^{d}$ inverts the light-cone ordering relation $: z_{1} \geq z_{2} \Leftrightarrow$ $\chi\left(z_{2}\right) \geq \chi\left(z_{1}\right)$.

We now introduce the following notations in which (for simplicity) we omit the dimension-dependent subscript used in the introduction (e.g. $X^{(c)}=X_{d-1}^{(c)}$, etc.) :

i) the complex hyperboloid $X^{(c)}=\left\{z \in \mathbb{C}^{d} ; z^{2}=-1\right\}$;

ii) the real one-sheeted hyperboloid $X=\mathbb{R}^{d} \cap X^{(c)}$ (see Figure 8);

iii) the "euclidean sphere" $S=\left(i \mathbb{R} \times \mathbb{R}^{d-1}\right) \cap X^{(c)}$ (see Figure 8);

iv) the point $z_{0}=(0,0, \ldots, 1)$ of $X \cap S$;

v) the following parametrization (П) of the set

$$
\begin{gathered}
\dot{X}^{(c)}=X^{(c)} \backslash\left\{z=\left(z^{(0)}, \vec{z}\right) \in \mathbb{C}^{d} ; z^{(d-1)^{2}}=1, z^{(0)} \neq 0\right\}, \\
z=z(\theta, \varphi, \vec{\alpha})=\left\{z^{(0)}=-i \sin \theta \cosh \varphi ; z^{(d-1)}=\cos \theta ;\right. \\
\left.[\vec{z}] \equiv\left(z^{(1)}, \ldots, z^{(d-2)}\right)=-i \sin \theta \sinh \varphi[\vec{\alpha}]\right\},
\end{gathered}
$$


with $\theta \in \mathbb{C}, \varphi \in \mathbb{C}, \vec{\alpha} \in \mathbb{S}_{(d-3)}^{(c)}$ (complex unit sphere), and the identifications :

$$
\begin{aligned}
& \text { (R) }(\theta, \varphi, \vec{\alpha}) \equiv(\theta+2 \pi, \varphi, \vec{\alpha}) \equiv(\theta, \varphi+2 i \pi, \vec{\alpha}) \\
& \equiv(-\theta, \varphi+i \pi, \vec{\alpha}) \equiv(\theta,-\varphi,-\vec{\alpha}) \text {. }
\end{aligned}
$$

In this representation, $S$ is obtained for $\theta=u \in \mathbb{R}, \varphi=i \eta, \eta \in \mathbb{R}$, $\vec{\alpha} \in \mathbb{S}_{(d-3)}$. The subset $\dot{X}=\dot{X}^{(c)} \cap \mathbb{R}^{d}$ of $X$ can be stratified as follows : $\dot{X}=Y \cup Y^{\prime} \cup Y^{\prime \prime}$, where

$$
\begin{aligned}
Y & =\left\{z \in X ; z^{(d-1)}>1\right\} \cup\left\{z_{0}\right\}=\left\{z \in \dot{X} ;\left(z-z_{0}\right)^{2} \geq 0\right\}, \\
Y^{\prime} & =\left\{z \in X ; z^{(d-1)}<1\right\} \cup\left\{-z_{0}\right\}=\left\{z \in \dot{X} ;\left(z+z_{0}\right)^{2} \geq 0\right\}, \\
Y^{\prime \prime} & =\left\{z \in X ;\left|z^{(d-1)}\right|<1\right\}=\left\{z \in \dot{X} ;\left(z-z_{0}\right)^{2}<0,\left(z+z_{0}\right)^{2}<0\right\} .
\end{aligned}
$$

In the parametrization ( $\Pi), Y$ and $Y^{\prime}$ are respectively obtained for $\theta=i v$ and $\theta=\pi+i v$, with $v, \varphi, \vec{\alpha}$ real and arbitrary. $Y^{\prime \prime}$ is obtained for $\theta=u \in \mathbb{R}, \varphi=\xi \pm i \frac{1}{2} \pi, \xi \in \mathbb{R}, \vec{\alpha} \in \mathbb{S}_{(d-3)}$.

If we now introduce the closed sets $X^{+}=\left\{z \in X ; z \geq z_{0}\right\}$ and $X^{-}=\chi\left(X^{+}\right)=\left\{z \in X ; z \leq z_{0}\right\}$, we then have the finer decomposition of the set $Y=\dot{X}^{+} \cup \dot{X}^{-} \cup\left\{z_{0}\right\}$, where the sets $\dot{X}^{+}=X^{+} \cap \dot{X}$ and $\dot{X}^{-}=X^{-} \cap \dot{X}$ are respectively obtained by taking $v>0$ and $v<0$ in the parametrization $(\Pi)$ of $Y$.

We now introduce the following groups of matrices acting on $\mathbb{C}^{d}$ :

i') $A^{(c)}=\{a(\theta) ; \theta \in \mathbb{C}\}$, where

$$
a(\theta)=\left(\begin{array}{ccc}
\cos \theta & 0 & -i \sin \theta \\
0 & I_{(d-2)} & 0 \\
-i \sin \theta & 0 & \cos \theta
\end{array}\right) ;
$$

ii $\left.^{\prime}\right) B^{(c)}=\{b(\varphi) ; \varphi \in \mathbb{C}\}$, where

$$
b(\varphi)=\left(\begin{array}{cccc}
\cosh \varphi & 0 & \sinh \varphi & 0 \\
0 & I_{(d-3)} & 0 & 0 \\
\sinh \varphi & 0 & \cosh \varphi & 0 \\
0 & 0 & 0 & 1
\end{array}\right) ;
$$

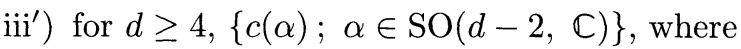

$$
c(\alpha)=\left(\begin{array}{ccc}
1 & 0 & 0 \\
0 & \alpha & 0 \\
0 & 0 & 1
\end{array}\right)
$$

TOME $120-1992-\mathrm{N}^{\circ} 2$ 
By using formulae (21), (22), (23) one readily checks that the parametric representation (19) can be rewritten as follows :

$$
\begin{aligned}
& \forall d \geq 3, \quad z(\theta, \varphi, \vec{\alpha})=\mathbf{m}_{\alpha \varphi \theta} z_{0} \\
& \text { where }: \mathbf{m}_{\alpha \varphi \theta}=c(\alpha) b(\varphi) a(\theta) .
\end{aligned}
$$

(Note that for $d=3, c(\alpha)$ can be chosen (in view of the relation $R$ ) equal to the unit matrix.)

\subsection{Kernels on $S$, Volterra kernels on $X$ and the composition} products $*$ and $\diamond$.

We represent $X^{(c)}$ by the equation $s(z)=0$, with

$$
s(z)=z^{(0)^{2}}-z^{(1)^{2}}-\cdots-z^{(d-1)^{2}}+1,
$$

and define the invariant measure on $X^{(c)}$ by the following $(d-1)$-form (with the notation of [10]) :

$$
\mathrm{d} \sigma^{(c)}(z)=\left.2 \frac{\mathrm{d} z^{(0)} \wedge \mathrm{d} z^{(1)} \wedge \cdots \wedge \mathrm{d} z^{(d-1)}}{i \mathrm{~d} s(z)}\right|_{X^{(c)}}
$$

We then introduce the real-valued measures : $\mathrm{d} \boldsymbol{\sigma}(z)=\left.\mathrm{d} \sigma^{(c)}(z)\right|_{S}$, $\mathrm{d} \boldsymbol{\sigma}(z)=-i \mathrm{~d} \sigma^{(c)}(z) \mid X$ and the following composition products $*$ and $\diamond$, respectively on $S$ and $X$.

With every kernel $\mathbf{K}\left(z, z^{\prime}\right)$ in $\mathcal{C}^{0}(S \times S)$ and function $\mathbf{F}(z)$ in $\mathcal{C}^{0}(S)$, we associate the function $\mathbf{G}=\mathbf{K} * \mathbf{F}$ in $\mathcal{C}^{0}(S)$ by the formula :

$$
\mathbf{G}(z)=\int_{S} \mathbf{K}\left(z, z^{\prime}\right) \mathbf{F}\left(z^{\prime}\right) \mathrm{d} \boldsymbol{\sigma}\left(z^{\prime}\right)
$$

Following [1], [2] we can similarly associate with every kernel $K\left(z, z^{\prime}\right)$ in $\mathcal{C}^{0}(X \times X)$ and function $F(z)$ in $\mathcal{C}^{0}(X)$, such that supp $K \subset \Sigma^{+}=$ $\left\{\left(z, z^{\prime}\right) \in X \times X ; z \geq z^{\prime}\right\}$ and $\operatorname{supp} F \subset X^{+}=\left\{z \in X ; z \geq z_{0}\right\}$, the following function $G=K \diamond F$ in $\mathcal{C}^{0}(X)$ by the formula :

$$
G(z)=\int_{\diamond\left(z, z_{0}\right)} K\left(z, z^{\prime}\right) F\left(z^{\prime}\right) \mathrm{d} \sigma\left(z^{\prime}\right)
$$

where $\diamond\left(z, z_{0}\right)$ represents the "double cone with diagonal $\left[z, z_{0}\right]$ on $X$ ", i.e. the compact set : $\left\{z^{\prime} \in X ; z \geq z^{\prime} \geq z_{0}\right\}$. 
Functions $K$ and $F$ satisfying the previous support conditions are respectively called (as in [1], [2]) Volterra kernels and Volterra functions on $X$. The function $G=K \diamond F$ associated with the couple $(K, F)$ is then itself a Volterra function.

We now wish to introduce Volterra functions and kernels with more special support properties, and for this purpose, we define for every $\mu$, $\mu \geq 0$, the following subsets of $X$ (see Figure 8) :

$$
\begin{aligned}
X_{\mu}^{+} & =\left\{z \in X ;\left(z-z_{0}\right)^{2} \geq \xi(\mu), z^{(0)} \geq 0\right\}, \\
& =\left\{z \in X^{+} ; z^{(d-1)} \geq \cosh \mu\right\} ; \\
X_{\mu}^{-} & =\chi\left(X_{\mu}^{+}\right)=\left\{z \in X^{-} ; z^{(d-1)} \geq \cosh \mu\right\}, \\
\text { with } \xi(\mu) & =2(\cosh \mu-1) .
\end{aligned}
$$

In the parametrization ( $\Pi$ ) (see Eq. (19)), $X_{\mu}^{+}$(resp. $X_{\mu}^{-}$) is obtained for $\theta=i v, v \geq \mu(\operatorname{resp} v \leq-\mu), \varphi \in \mathbb{R}$ and $\vec{\alpha} \in \mathbb{S}_{(d-3)}$. Now we denote by $V_{\mu}$ the class of functions $F(z)$ in $\mathcal{C}^{0}(X)$ satisfying the support condition : $\operatorname{supp} F \subset X_{\mu}^{+}$. Similarly we consider the sets :

$$
\Sigma_{\mu}^{ \pm}=\left\{\left(z, z^{\prime}\right) \in X \times X ;\left(z-z^{\prime}\right)^{2} \geq \xi(\mu), z^{(0)}-z^{(0)} \gtrless 0\right\} .
$$

Then we denote by $W_{\mu}$ the class of kernels $K\left(z, z^{\prime}\right)$ in $\mathcal{C}^{(0)}(X \times X)$ satisfying the condition : $\operatorname{supp} K \subset \Sigma_{\mu}^{+}$.

The following property holds :

Proposition 4. - For every kernel $K$ in $W_{\mu_{1}}$ and every function $F$ in $V_{\mu_{2}}$, the corresponding function $G=K \diamond F$ (defined by Eq. (27)) belongs to $V_{\mu_{1}+\mu_{2}}$.

Proof. - One must check that the conditions $z^{\prime} \in X_{\mu_{2}}^{+},\left(z, z^{\prime}\right) \in \Sigma_{\mu_{1}}^{+}$ imply $z \in X_{\mu_{1}+\mu_{2}}^{+}$. The points $z, z^{\prime}$ being such that $z \geq z^{\prime} \geq z_{0}$, the representation ( $\Pi$ ) (see Eq. (19)) of $z, z^{\prime}$ is given in terms of real "hyperbolic angles" $v(\theta=i v), v^{\prime}\left(\theta^{\prime}=i v^{\prime}\right), \varphi, \varphi^{\prime}$ (with $\vec{\alpha}, \vec{\alpha}^{\prime}$ in $\left.S_{(d-3)}\right)$. We then obtain for the minkowskian scalar product of $z$ and $z^{\prime}$ the expression :

$$
\begin{gathered}
z \cdot z^{\prime}=\sinh v \sinh v^{\prime}\left[\cosh \varphi \cosh \varphi^{\prime}-\sinh \varphi \sinh \varphi^{\prime} \vec{\alpha} \cdot \vec{\alpha}^{\prime}\right] \\
-\cosh v \cosh v^{\prime}
\end{gathered}
$$

If $z \geq z^{\prime}$, one has : $\left(z-z^{\prime}\right)^{2}=-2\left(1+z \cdot z^{\prime}\right) \geq 0$ and the hyperbolic angle $V$ between $z$ and $z^{\prime}$ can be defined by $\cosh V=-z \cdot z^{\prime}$; Eq. (30)

TOME $120-1992-\mathrm{N}^{\circ} 2$ 
then yields the following "hyperbolic triangular inequality", valid for all points $z, z^{\prime}$ in $X$ such that $z \geq z^{\prime} \geq z_{0}: V \leq v-v^{\prime}$. Since (in view of (28), (29)), the conditions $z^{\prime} \in X_{\mu_{2}}^{+},\left(z, z^{\prime}\right) \in \Sigma_{\mu_{1}}^{+}$are respectively equivalent to $-z^{\prime} \cdot z_{0} \geq \cosh \mu_{2}$ and $-z \cdot z^{\prime} \geq \cosh \mu_{1}$ (with $z \geq z^{\prime} \geq z_{0}$ ), i.e. $v^{\prime} \geq \mu_{2}$ and $V \geq \mu_{1}$, it follows that $v \geq V+v^{\prime} \geq \mu_{1}+\mu_{2}$, and therefore $z \in X_{\mu_{1}+\mu_{2}}^{+}$.

Similarly, we shall introduce the composition products $*$ and $\diamond$, respectively for kernels on $S$ and Volterra kernels on $X$ :

- With every couple $\left(\mathbf{K}_{1}, \mathbf{K}_{2}\right)$ with $\mathbf{K}_{i} \in \mathcal{C}^{0}(S \times S)(i=1,2)$, we associate the kernel :

$$
\mathbf{K}\left(z, z^{\prime}\right)=\left(\mathbf{K}_{1} * \mathbf{K}_{2}\right)\left(z, z^{\prime}\right) \equiv \int_{S} \mathbf{K}_{1}\left(z, z^{\prime \prime}\right) \mathbf{K}_{2}\left(z^{\prime \prime}, z^{\prime}\right) \mathbf{d} \boldsymbol{\sigma}\left(z^{\prime \prime}\right) .
$$

- With every couple of Volterra kernels $\left(K_{1}, K_{2}\right)$ on $X$, we associate (as in [1], [2]) the kernel :

$$
K\left(z, z^{\prime}\right)=\left(K_{1} \diamond K_{2}\right)\left(z, z^{\prime}\right)=\int_{\diamond\left(z, z^{\prime}\right)} K_{1}\left(z, z^{\prime \prime}\right) K_{2}\left(z^{\prime \prime}, z^{\prime}\right) \mathrm{d} \sigma\left(z^{\prime \prime}\right),
$$

where $\diamond\left(z, z^{\prime}\right)$ denotes the compact set $\left\{z^{\prime \prime} \in X ; z \geq z^{\prime \prime} \geq z^{\prime}\right\}$.

For all $z^{\prime}$ in $X^{+}$, we can write (in view of Eq. (24)) : $z^{\prime}=\mathbf{m}_{\alpha^{\prime} \varphi^{\prime} \theta^{\prime}} z_{0}$ with $\alpha^{\prime} \in \mathrm{SO}(d-2 ; R), \varphi^{\prime} \in \mathbb{R}, \theta^{\prime} \in i \mathbb{R}^{+}$, and introduce the point $Z=\mathbf{m}_{\alpha^{\prime} \varphi^{\prime} \theta^{\prime}}^{-1} z$. This matrix $\mathbf{m}_{\alpha^{\prime} \varphi^{\prime} \theta^{\prime}}$ preserves the order relation $\geq$ on $X$ and therefore the following formula holds : $\diamond\left(z, z^{\prime}\right)=\mathbf{m}_{\alpha^{\prime} \varphi^{\prime} \theta^{\prime}}\left[\diamond\left(Z, z_{0}\right)\right]$.

Since the measure $\mathrm{d} \sigma$ is invariant under the transformation $Z^{\prime \prime} \mapsto z^{\prime \prime}=\mathbf{m}_{\alpha^{\prime} \varphi^{\prime} \theta^{\prime}} Z^{\prime \prime}$, we can write Eq. (32) as follows :

$\left(32^{\prime}\right) \quad K\left(\mathbf{m}_{\alpha^{\prime} \varphi^{\prime} \theta^{\prime}} Z, \mathbf{m}_{\alpha^{\prime} \varphi^{\prime} \theta^{\prime}} z_{0}\right)$

$$
\begin{aligned}
=\int_{\diamond\left(Z, z_{0}\right)} K_{1}\left(\mathbf{m}_{\alpha^{\prime} \varphi^{\prime} \theta^{\prime}} Z, \mathbf{m}_{\alpha^{\prime} \varphi^{\prime} \theta^{\prime}} Z^{\prime \prime}\right) \\
\\
\times K_{2}\left(\mathbf{m}_{\alpha^{\prime} \varphi^{\prime} \theta^{\prime}} Z^{\prime \prime}, \mathbf{m}_{\alpha^{\prime} \varphi^{\prime} \theta^{\prime}} z_{0}\right) \mathrm{d} \sigma\left(Z^{\prime \prime}\right) .
\end{aligned}
$$

Now since

$$
\begin{aligned}
& \Sigma_{\mu_{i}}^{+}=\left\{\left(z, z^{\prime}\right) \in\right. X \times X ; z=\mathbf{m}_{\alpha^{\prime} \varphi^{\prime} \theta^{\prime}} Z, z^{\prime}=\mathbf{m}_{\alpha^{\prime} \varphi^{\prime} \theta^{\prime}} z_{0} \\
&\left.\alpha^{\prime} \in \mathrm{SO}(d-2 ; R), \varphi^{\prime} \in \mathbb{R}, \theta^{\prime} \in i \mathbb{R}, Z \in X_{\mu_{i}}^{+}\right\}
\end{aligned}
$$

(with $i=1,2$ ) the assumptions of Proposition 4 are satisfied by the integrand at the r.h.s. of formula $\left(32^{\prime}\right)$ (which is then of the form of Eq. (27)); therefore the function $\mathcal{G}(Z) \equiv K\left(\mathbf{m}_{\alpha^{\prime} \varphi^{\prime} \theta^{\prime}} Z, \mathbf{m}_{\alpha^{\prime} \varphi^{\prime} \theta^{\prime}} z_{0}\right)$ belongs to the class $V_{\mu_{1}+\mu_{2}}$ which (in view of Eq. $\left(32^{\prime \prime}\right)$, written for $\Sigma_{\mu_{1}+\mu_{2}}^{+}$ and $\left.X_{\mu_{1}+\mu_{2}}^{+}\right)$implies that $K\left(z, z^{\prime}\right)$ belongs to the class $W_{\mu_{1}+\mu_{2}}$. We can thus state : 
Proposition 5. - For every couple of Volterra kernels $\left(K_{1}, K_{2}\right)$ with $K_{i} \in W_{\mu_{i}}(i=1,2)$, the composition product $K=K_{1} \diamond K_{2}$ is a Volterra kernel in $W_{\mu_{1}+\mu_{2}}$.

\subsection{Holomorphic functions and perikernels on $X^{(c)}$.}

We introduce the following subsets of $X^{(c)}$ :

i) the "cut"

$$
\begin{aligned}
\Xi_{\mu} & =\left\{z \in X^{(c)} ;\left(z-z_{0}\right)^{2}-\xi(\mu) \geq 0\right\} \\
& =\left\{z \in X^{(c)} ; z^{(d-1)}=\cosh v ; v \geq \mu\right\}
\end{aligned}
$$

ii) the "cut-domain" $D_{\mu}=X^{(c)} \backslash \Xi_{\mu}$. We also put $\dot{D}_{\mu}=\dot{X}^{(c)} \backslash \Xi_{\mu}$.

Analogously we introduce in $X^{(c)} \times X^{(c)}$ the following subsets :

$\left.\mathrm{i}^{\prime}\right)$ the "cut" $\Sigma_{\mu}^{(c)}=\left\{\left(z, z^{\prime}\right) \in X^{(c)} \times X^{(c)} ;\left(z-z^{\prime}\right)^{2}-\xi(\mu) \geq 0\right\}$;

ii') the "cut-domain" $\Delta_{\mu}=\left(X^{(c)} \times X^{(c)}\right) \backslash \Sigma_{\mu}^{(c)}$. We shall also put $\dot{\Delta}_{\mu}=\left(\dot{X}^{(c)} \times \dot{X}^{(c)}\right) \backslash \Sigma_{\mu}^{(c)}$.

We denote by $V_{\mu}^{(c)}$ the subset of $V_{\mu}$ whose elements $F(z)$ are moreover analytic (with respect to $(\varphi, \vec{\alpha})$, via $z=z(\theta, \varphi, \vec{\alpha})$ ) in the full manifold $\Xi_{\mu}$. Similarly we denote by $W_{\mu}^{(c)}$ the subset of $W_{\mu}$ whose elements $K\left(z, z^{\prime}\right)$ have analytic continuation in the full manifold $\Sigma_{\mu}^{(c)}$.

We now introduce the following classes of functions :

a) $\mathcal{V}_{\mu}$ denotes the class of holomorphic functions $\mathcal{F}(z)$ in $D_{\mu}$ which are moreover continuous on the boundary set $\Xi_{\mu}$ (from both sides of this "cut");

b) $\mathcal{W}_{\mu}$ denotes the class of functions $\mathcal{K}\left(z, z^{\prime}\right)$ which are holomorphic in $\Delta_{\mu}$ and continuous on the boundary sets $\Sigma_{\mu}^{(c)}$ (from both sides of this "cut"). Such functions will be called "perikernels" on $X^{(c)}$.

Variant. - One can release the continuity condition on the boundaries and replace it by a "slow increase condition", corresponding to the existence of boundary values in the sense of distributions; for instance, for any integer $s(s>0)$ one can define the spaces $\mathcal{V}_{\mu, s}$ and $\mathcal{W}_{\mu, s}$ of holomorphic functions $\mathcal{F}(z)$ and $\mathcal{K}\left(z, z^{\prime}\right)$ which are derivatives of total order $s$ of functions belonging respectively to the previous spaces $\mathcal{V}_{\mu}$ and $\mathcal{W}_{\mu}$.

Now, for every function $\mathcal{F}$ in $\mathcal{V}_{\mu}$, we shall put $\mathbf{F}=\mathcal{F}_{\mid S}$ (F belongs to $\mathcal{C}^{0}(S)$ ) and we consider the associated "discontinuity function"

TOME $120-1992-\mathrm{N}^{\circ} 2$ 
$\Delta \mathcal{F}=i\left(\mathcal{F}_{+}-\mathcal{F}_{-}\right)$of $\mathcal{F}$, where $\mathcal{F}_{+}$and $\mathcal{F}_{-}$denote the boundary values of $\mathcal{F}$ on the analytic hypersurface $\left\{z \in X^{(c)} ; \operatorname{Im}\left(z-z_{0}\right)^{2}=0\right\}$ from the respective sides $\operatorname{Im}\left(z-z_{0}\right)^{2}<0$ and $\operatorname{Im}\left(z-z_{0}\right)^{2}>0$. We note that, in the set $\dot{X}^{+}=\left\{z \in \dot{X} ; z \geq z_{0}\right\}$, parametrized (in view of Eq. (19)) by

$$
\left\{(v, \varphi, \vec{\alpha}) ; v \geq 0, \varphi \in \mathbb{C}, \vec{\alpha} \in \mathbb{S}_{d-3}^{(c)}\right\}
$$

one can write $\left(\right.$ since $\operatorname{Im}\left(z-z_{0}\right)^{2}=2 \operatorname{Im} \cos (\varepsilon+i v)<0$, for $\left.\varepsilon>0\right)$ :

$$
\mathcal{F}_{ \pm}\{z\}=\lim _{\substack{\varepsilon \rightarrow 0 \\ \varepsilon>0}} \mathcal{F}[z(i v \pm \varepsilon, \varphi, \vec{\alpha})]
$$

The function $\Delta \mathcal{F}$ has its support contained in the set $\Xi_{\mu}$ and is analytic on the complex submanifolds $\left(z-z_{0}\right)^{2}=\rho, \rho \geq \xi(\mu)$ (parametrized by $\left.v=v(\rho)=\cosh ^{-1}\left[1+\frac{1}{2} \rho\right], \varphi \in \mathbb{C}, \vec{\alpha} \in \mathbb{S}_{d-3}^{(c)}\right)$ which generate this set. The corresponding function $F(z)=\Delta_{+} \mathcal{F}(z)=Y\left(z^{(0)}\right) \Delta \mathcal{F}_{\mid X}(z)$ (where $Y$ denotes the Heaviside function) has its support contained in the set $X_{\mu}^{+}$ and belongs to the class $V_{\mu}^{(c)}$. One can also define in an analogous way the function $\Delta_{-} \mathcal{F}^{0}(z)=Y\left(-z^{(0)}\right) \Delta \mathcal{F}_{\mid X}(z)$ whose support is contained in the set $X_{\mu}^{-}$.

Similarly, for every kernel $\mathcal{K}$ in $\mathcal{W}_{\mu}$, we put $\mathbf{K}=\mathcal{K}_{\mid S \times S}$ (K belongs to $\mathcal{C}^{0}(S \times S)$ ) and we consider the associated "discontinuity function" $\Delta \mathcal{K}=i\left(\mathcal{K}_{+}-\mathcal{K}_{-}\right)$of $\mathcal{K}$, where $\mathcal{K}_{+}$and $\mathcal{K}_{-}$denote the boundary values of $\mathcal{K}$ on the analytic hypersurface $\left\{\left(z, z^{\prime}\right) \in X^{(c)} \times X^{(c)} ; \operatorname{Im}\left(z-z^{\prime}\right)^{2}=0\right\}$ from the respective sides $\operatorname{Im}\left(z-z^{\prime}\right)^{2}<0$ and $\operatorname{Im}\left(z-z^{\prime}\right)^{2}>0$. The function $\Delta \mathcal{K}$ has its support contained in the set $\Sigma_{\mu}^{(c)}$ and is analytic on the complex submanifolds $\left(z-z^{\prime}\right)^{2}=\rho, \rho \geq \xi(\mu)$ which generate this set. The corresponding function

$$
K\left(z, z^{\prime}\right)=\Delta_{+} \mathcal{K}\left(z, z^{\prime}\right)=Y\left(z^{(0)}-z^{(0)}\right) \Delta \mathcal{K}_{\mid X \times X}\left(z, z^{\prime}\right)
$$

is then a Volterra kernel on $X$ with support contained in $\Sigma_{\mu}^{+}$, which belongs to the class $W_{\mu}^{(c)}$. One can also define similarly the function $\Delta_{-} \mathcal{K}\left(z, z^{\prime}\right)=Y\left(z^{\prime(0)}-z^{(0)}\right) \Delta \mathcal{K}_{\mid X \times X}\left(z, z^{\prime}\right)$ whose support is contained in $\Sigma_{\mu}^{-}$.

The study of a general composition product $*^{(c)}$ for perikernels will be presented below in section 3 and will provide results similar to those obtained for the classes of perikernels $\mathcal{P}_{\mu}\left(X^{(c)}\right)$ and $\widetilde{\mathcal{P}}_{\mu}\left(X^{(c)}\right)$ of the case $d=2$ (see Proposition 3 and $\S 1.3$ ). 
Remark. - Comparing the set of perikernels $\mathcal{W}_{\mu}$ with the set $\widetilde{\mathcal{P}}_{\mu}\left(X^{(c)}\right)$, of the case $d=2$, calls for the following comment. In the case $d=2$, the analogue of the holomorphy domain of the kernels in $\mathcal{W}_{\mu}$ can be described as $X^{(c)} \times X^{(c)} \backslash\left(\Sigma_{\mu}^{(c)_{+}} \cup \Sigma_{\mu}^{(c)}{ }_{-}\right)$, where $\Sigma_{\mu}^{(c)_{-}}=\left\{\left(z, z^{\prime}\right) ;\left(z^{\prime}, z\right) \in \Sigma_{\mu}^{(c)}{ }_{+}\right\}$. We then notice that the holomorphy domain $\left(X^{(c)} \times X^{(c)}\right) \backslash \Sigma_{\mu}^{(c)}+$ of the kernels in $\widetilde{\mathcal{P}}_{\mu}\left(X^{c}\right)$ has no counterpart in the general case $(d \geq 3)$, since $\Sigma_{\mu}^{(c)}+$ and $\Sigma_{\mu}^{(c)}{ }_{-}$then become a single analytic hypersurface $\Sigma_{\mu}^{(c)}$ (due to the extravariables $\varphi$ and $[\vec{\alpha}])$.

\section{The composition product $*^{(c)}$ for perikernels on $X^{(c)}$}

\subsection{From the sphere to the hyperboloid : the analytic continu- ation procedure.}

We will establish the following result, valid for an arbitrary dimension $d$, with $d \geq 3$.

Theorem 1. - Let $\mathcal{K}$ belong to $\mathcal{W}_{\mu_{1}}$, and $\mathcal{F}$ belong to $\mathcal{V}_{\mu_{2}}$. Then there exists a unique function $\mathcal{G}$ in the class $\mathcal{V}_{\mu}$, with $\mu=\mu_{1}+\mu_{2}$, denoted by $\mathcal{K} *^{(c)} \mathcal{F}$ which satisfies the following properties :

a)

$$
\mathcal{G}_{\mid S}=\mathcal{K}_{\mid S \times S} * \mathcal{F}_{\mid S}
$$

b)

$$
\Delta_{+} \mathcal{G}=\Delta_{+} \mathcal{K} \diamond \Delta_{+} \mathcal{F}
$$

c) For every point $z$ in $\dot{D}_{\mu}$, there exists a class of cycles $\dot{\Gamma}(z)$ in $H^{(d-1)}\left(D_{\mu_{1} \mu_{2}}^{z}\right)$, where $D_{\mu_{1} \mu_{2}}^{z}=\left\{z^{\prime} \in X^{(c)} ; z^{\prime} \in \dot{D}_{\mu_{2}},\left(z, z^{\prime}\right) \in \dot{\Delta}_{\mu_{1}}\right\}$, such that:

$$
\mathcal{G}(z)=\int_{\dot{\Gamma}(z)} \mathcal{K}\left(z, z^{\prime}\right) \mathcal{F}\left(z^{\prime}\right) \mathrm{d} \sigma^{(c)}\left(z^{\prime}\right)
$$

The main part of the proof consists in constructing a representative cycle $\Gamma(z)$ of $\dot{\Gamma}(z)$ (relevant for formula (37)) and proving formulae (35), (36) for all points $z$ in the section $D_{\mu, 0}$ of $D_{\mu}$ by the $\left(z^{(0)}, z^{(d-1)}\right)$ plane $\left(\left(z^{0}, z^{(d-1)}\right) \in \mathbb{C}^{2}\right)$, as specified in Lemma 1 stated below. In this statement, it will appear that the general $d$-dimensional case reduces trivially to the 3-dimensional case. The remaining part of the proof of 
Theorem 1, namely the extension of the results of Lemma 1 to all points $z$ in $D_{\mu}$, relies on short arguments of group theory and several complex variable analysis; it will be given before the (longer) proof of LEMMA 1.

Let

$$
X_{0}^{(c)}=\left\{z=\left(z^{(0)},[\overrightarrow{0}], z^{(d-1)}\right) ; z^{(0)}=-i \sin \theta, z^{(d-1)}=\cos \theta, \theta \in \mathbb{C}\right\} .
$$

The domain $D_{\mu, 0}=D_{\mu} \cap X_{0}^{(c)}$ of $X_{0}^{(c)}$ is then represented in the $\theta$-plane by the set $\dot{\mathcal{J}}_{\mu}=\mathcal{J}_{\mu} / 2 \pi \mathbb{Z}$, with

$$
\mathcal{J}_{\mu}=\{\theta \in \mathbb{C} ; \theta \neq i v+2 k \pi,|v| \geq \mu, k \in \mathbb{Z}\} ;
$$

we also introduce the sections $S_{0}, X_{\mu, 0}^{ \pm}$of the sets $S, X_{\mu}^{ \pm}$by $X_{0}^{(c)}$, represented in the $\theta$-plane respectively by the sets $\{\theta \in \mathbb{R}\}$,

$$
\{\theta=i v(\bmod 2 \pi) ; \pm v \geq \mu\} .
$$

For $z=z(\theta)=(-i \sin \theta, \overrightarrow{0}, \cos \theta)$ in $D_{\mu, 0}$, we shall define the integral (37) by using the parametrization (П) for the integration variables $z^{\prime}$, namely by putting $z^{\prime}=z^{\prime}\left(\theta^{\prime}, \varphi^{\prime}, \vec{\alpha}^{\prime}\right)$ according to formulae (19), with $\left(\theta^{\prime}, \varphi^{\prime}, \vec{\alpha}^{\prime}\right)$ varying in $\mathbb{C}^{2} \times \mathbb{S}_{d-3}^{(c)} / R$ (see Eq. (20)). This allows us to represent a dense subdomain of the analyticity fiber of the integrand $D_{\mu_{1} \mu_{2}}^{z(\theta)}=\left\{z^{\prime} \in \dot{X}^{(c)} ; z^{\prime} \in D_{\mu_{2}},\left(z(\theta), z^{\prime}\right) \in \Delta_{\mu_{1}}\right\}$ by the following "cutdomain" (in view of the definitions i), ii), $\mathrm{i}^{\prime}$ ), ii ${ }^{\prime}$ ) in $\S 2.3$ ) :

$$
D\{\theta\}=\left\{\left(\theta^{\prime}, \varphi^{\prime}\right) \in \mathbb{C}^{2} ;\left(\theta^{\prime}, \varphi^{\prime}\right) \notin \Xi\left(\mu_{2}\right) \cup \Sigma\left(\mu_{1} ; \theta\right)\right\} \times \mathbb{S}_{d-3}^{(c)} / R
$$

where :

$$
\Xi\left(\mu_{2}\right)=\left\{\left(\theta^{\prime}, \varphi^{\prime}\right) \in \mathbb{C}^{2} ; \theta^{\prime}=i v^{\prime}+2 k \pi,\left|v^{\prime}\right| \geq \mu_{2}, k \in \mathbb{Z}\right\}
$$

and

$$
\begin{aligned}
\Sigma\left(\mu_{1} ; \theta\right)=\left\{\left(\theta^{\prime}, \varphi^{\prime}\right) \in \mathbb{C}^{2} ; \cos \theta \cos \theta^{\prime}+\sin \theta\right. & \sin \theta^{\prime} \cosh \varphi^{\prime} \\
& \left.\geq \cosh \mu_{1}=\rho_{1}\right\} .
\end{aligned}
$$

Formula (40) is obtained by noticing that (in view of the representation $(\Pi)$, one has : $z\{\theta\} \cdot z^{\prime}\left(\theta^{\prime}, \varphi^{\prime}, \vec{\alpha}^{\prime}\right)=-\left(\sin \theta \sin \theta^{\prime} \cosh \varphi^{\prime}+\cos \theta \cos \theta^{\prime}\right)$.

We can then state the following basic property, in which the integration measure (involved in Eq. (41)) is the parametric expression of $\mathrm{d} \sigma^{(c)}\left(z^{\prime}\right)$ (see Eq. (25)). 
Lemma 1. - For every point $z=z(\theta)$ in $D_{\mu, 0}$ one can define the analytic function $\mathcal{G}(z)$ of theorem 1 by an integral of the following form :

$$
\begin{aligned}
\mathcal{G}(z)=(-i)^{d-2} \int_{\Gamma_{\theta}} \mathcal{K}\left(z, z^{\prime}\left(\theta^{\prime}, \varphi^{\prime}, \vec{\alpha}^{\prime}\right)\right) \mathcal{F}\left(z^{\prime}\left(\theta^{\prime}, \varphi^{\prime}, \vec{\alpha}^{\prime}\right)\right) \\
\\
\left(\sin \theta^{\prime}\right)^{d-2}\left(\sinh \varphi^{\prime}\right)^{d-3} \mathrm{~d} \theta^{\prime} \wedge \omega\left(\vec{\alpha}^{\prime}\right) \wedge \mathrm{d} \varphi^{\prime}
\end{aligned}
$$

where $\Gamma_{\theta}$ is a $(d-1)$-cycle whose support is a compact subset of $D(\theta)$ of the form $\left\{\left(\theta^{\prime}, \varphi^{\prime}, \vec{\alpha}^{\prime}\right) ; \theta^{\prime} \in \gamma_{\theta}, \varphi^{\prime} \in L_{\theta \theta^{\prime}}, \vec{\alpha}^{\prime} \in \mathbb{S}_{d-3}\right\}, \gamma_{\theta}$ and $L_{\theta \theta^{\prime}}$ being appropriate paths depending continuously on $\theta$ and $\left(\theta, \theta^{\prime}\right)$ respectively, and where $\omega\left(\vec{\alpha}^{\prime}\right)$ denotes the $(\mathrm{SO}(d-2)$-invariant $)$ unit volume form on $\mathbb{S}_{d-3}$.

For $z=z(\theta)$ in $S_{0}$, the integral (41) reduces to the expression $\mathcal{K}_{\mid S_{0} \times S} * \mathcal{F}_{\mid S}$, obtained for $\gamma_{\theta}=\mathbb{R} / 2 \pi \mathbb{Z}$ and $L_{\theta \theta^{\prime}}=i \mathbb{R}(\bmod 2 \pi) ;$ for $z(\theta)$ in $X_{\mu, 0}^{+}$, the corresponding discontinuity $\Delta_{+} \mathcal{G}$ is given by formula (36).

Starting from this result, the proof of THEOREM 1 is achieved as follows. In view of formula (24), one can write for each point $z=z(\theta, \varphi, \vec{\alpha})$ in $\dot{X}^{(c)}$ :

$$
z(\theta, \varphi, \vec{\alpha})=c(\alpha) b(\varphi) a(\theta) z_{0}=c(\alpha) b(\varphi) z(\theta)
$$

with

$$
z(\theta)=a(\theta) z_{0}=(-i \sin \theta, \overrightarrow{0}, \cos \theta) \in X_{0}^{(c)}
$$

Moreover $z(\theta, \varphi, \vec{\alpha})$ belongs to the domain $\dot{D}_{\mu}$ if and only if $z(\theta)$ belongs to $D_{\mu, 0}$, since one easily checks that $\left(z(\theta, \varphi, \vec{\alpha})-z_{0}\right)^{2}=\left(z(\theta)-z_{0}\right)^{2}$.

For any point $z=z(\theta, \varphi, \vec{\alpha})$ in $\dot{D}_{\mu}$, we then put :

$$
\mathcal{G}(z)=\int_{\Gamma(z)} \mathcal{K}\left(z, z^{\prime}\right) \mathcal{F}\left(z^{\prime}\right) \mathrm{d} \sigma^{(c)}\left(z^{\prime}\right)
$$

with $\Gamma(z(\theta, \varphi, \vec{\alpha}))=c(\alpha) b(\varphi) \Gamma_{\theta}$.

We shall first show that this function belongs to the class $\mathcal{V}_{\mu}$, with $\mu=\mu_{1}+\mu_{2}$. In view of the invariance of the domain $D_{\mu_{2}}$ (resp. $\Delta_{\mu_{1}}$ ) of $\mathcal{F}$ (resp. $\mathcal{K}$ ) under the set of transformations $z^{\prime} \mapsto c(\alpha) b(\varphi) z^{\prime}$ (resp. $\left(z, z^{\prime}\right) \mapsto\left(c(\alpha) b(\varphi) z, c(\alpha) b(\varphi) z^{\prime}\right)$, it follows that the support of the $(d-1)$-cycle $\Gamma(z(\theta, \varphi, \vec{\alpha}))$ is compactly contained in the analyticity fiber $D_{\mu_{1}, \mu_{2}}^{z(\theta, \vec{\alpha})}=\left\{z^{\prime} \in X^{(c)} ; z^{\prime} \in D_{\mu_{2}},\left(z(\theta, \varphi, \vec{\alpha}), z^{\prime}\right) \in \Delta_{\mu_{1}}\right\}$ of the integrand of (44) at $z=z(\theta, \varphi, \vec{\alpha})$. Since Eq. (41) is defined in $D_{\mu, 0}$, the integral (44) defines a germ of analytic function at each point $z=z(\theta, \varphi, \vec{\alpha})$ in $\dot{D}_{\mu}$, and

TOME $120-1992-\mathrm{N}^{\circ} 2$ 
since the cycle $\Gamma(z(\theta, \varphi, \vec{\alpha}))$ varies continuously with $z$ in the analyticity domain $\mathcal{D}$ of $\mathcal{K}\left(z, z^{\prime}\right) \mathcal{F}\left(z^{\prime}\right)$ (namely defines, in the sense of [7] or [8] a continuous section of the homology bundle associated with $\mathcal{D}$, considered as a fiberbundle over $\dot{D}_{\mu}$ ), all these germs define $\mathcal{G}$ as an analytic function in $\dot{D}_{\mu}$. The extension of formula (35) from $S_{0}$ to $S$ follows directly from the invariance of $S$ and of the measure $\mathrm{d} \boldsymbol{\sigma}$ under the transformations $z^{\prime} \mapsto c(\alpha) b(\varphi) z^{\prime}$, for all $\alpha \in \mathrm{SO}(d-2 ; R), \varphi \in i \mathbb{R}, z^{\prime} \in S$, and from the fact that

$$
S=\left\{z=c(\alpha) b(\varphi) z(\theta) ; \alpha \in \operatorname{SO}(d-2 ; R), \varphi \in i \mathbb{R}, z(\theta) \in S_{0}\right\}
$$

The extension of formula (36) from $X_{\mu, 0}^{+}$to $X_{\mu}^{+}$follows from the invariance of the measure $\mathrm{d} \sigma$ under the transformations $z^{\prime} \mapsto c(\alpha) b(\varphi) z^{\prime}, \alpha \in$ $\mathrm{SO}(d-2 ; \mathbb{R}), \varphi \in \mathbb{R}, z^{\prime} \in X^{+}$and from the following properties :

$$
\begin{array}{r}
\forall \alpha \in \mathrm{SO}(d-2 ; R), \forall \varphi \in \mathbb{R}, \forall z=z(\theta, \varphi, \vec{\alpha}), \\
\diamond\left(z, z_{0}\right)=c(\alpha) b(\varphi)\left[\diamond\left(z(\theta), z_{0}\right)\right]
\end{array}
$$

and $X_{\mu}^{+}=\left\{z=c(\alpha) b(\varphi) z(\theta) ; \alpha \in \mathrm{SO}(d-2 ; R), \varphi \in \mathbb{R}, z(\theta) \in X_{\mu, 0}^{+}\right\}$.

Finally, it remains to establish that the function $\mathcal{G}(z)$ admits an analytic continuation on the $(d-2)$-dimensional subset $D_{\mu} \backslash \dot{X}^{(c)}$ of $X^{(c)}$, namely on the two cones $\mathcal{C}_{+}=\left\{z \in X^{(c)} ; z^{(d-1)}=1, z \neq z_{0}\right\}$ and $\mathcal{C}_{-}=\left\{z \in X^{(c)} ; z^{(d-1)}=-1, z \neq-z_{0}\right\}$, which are not represented by the parametrization ( $\Pi)$. From formula (41) written at $z=z_{0}($ i.e. $\theta=0)$, we know that $\mathcal{G}(z)$ is analytic in a neighbourhood $\mathcal{N}\left(z_{0}\right)$ of $z_{0}$ in $X^{(c)}$ and therefore in a certain open subset $\mathcal{N}^{\prime}=\mathcal{N}\left(z_{0}\right) \cap \mathcal{C}_{+}$of $\mathcal{C}_{+}$. It then follows from Bremermann's continuity theorem [11], applied to any family of onedimensional sections of $X^{(c)}$ admitting as its limit an arbitrary linear submanifold of $\mathcal{C}_{+}$(which is adherent to $z_{0}$ and has therefore a non-empty intersection with $\mathcal{N}^{\prime}$ ) that the whole set $\mathcal{C}_{+}$belongs to the analyticity domain of $\mathcal{G}$ (the set $\mathcal{C}_{-}$being treated similarly). The uniqueness of the function $\mathcal{G}$ satisfying the theorem is obvious since it is the analytic continuation of the r.h.s. of Eq. (35) ( $S$ being a $(d-1)$-dimensional real submanifold of $D_{\mu}$ ).

\section{Proof of Lemma 1 :}

\section{A) Construction of the cycle $\Gamma_{\theta}$}

We first specify the prescriptions to be satisfied by the cycle $\Gamma_{\theta}$, i.e. more precisely by the fiber bundle $\bigcup_{\theta^{\prime} \in \gamma_{\theta}}\left(\theta^{\prime}, L_{\theta \theta^{\prime}}\right)$, for $\theta$ belonging to the cut-plane $\mathcal{J}_{\mu}$. As a matter of fact, it will be often convenient to construct 
the image $\mathbf{L}_{\theta \theta^{\prime}}$ of $L_{\theta \theta^{\prime}}$ in the complex plane of the variable $\zeta=\cosh \varphi^{\prime}$. More precisely, we will use the biholomorphic mapping between the strip $\left\{\varphi^{\prime} \in \mathbb{C} ; 0<\operatorname{Im} \varphi^{\prime}<\pi\right\}$ and the cut-plane

$$
\left\{\zeta \in \mathbb{C} ; \zeta-1 \notin \mathbb{R}^{+},-\zeta-1 \notin \mathbb{R}^{+}\right\} .
$$

$L_{\theta \theta^{\prime}}$ and $\mathbf{L}_{\theta \theta^{\prime}}$ will always be respectively contained in these sets (or exceptionally on their boundaries).

a) The requirement that $\operatorname{supp} \Gamma_{\theta}$ should avoid the set $\Xi\left(\mu_{2}\right) \times \mathbb{S}_{d-3}^{(c)} / R$ corresponds (in view of formula (39)), to the following condition : in the $\theta^{\prime}$-plane, the contour $\gamma_{\theta}$ should always avoid the set

$$
\delta_{2}=\left\{\theta^{\prime}=i v^{\prime}+2 k \pi ;\left|v^{\prime}\right| \geq \mu_{2}, k \in \mathbb{Z}\right\} .
$$

b) The requirement that $\operatorname{supp} \Gamma_{\theta}$ should avoid the set

$$
\Sigma\left(\mu_{1} ; \theta\right) \times \mathbb{S}_{d-3}^{(c)} / R
$$

corresponds (in view of formula (40)) to a condition for the contour $L_{\theta \theta^{\prime}}$ in the $\varphi^{\prime}$-plane to be satisfied for each $\theta$ in $\mathcal{J}_{\mu}$ and $\theta^{\prime}$ in $\gamma_{\theta}$; this condition, more conveniently expressed in the $\zeta$-plane, is that $\mathbf{L}_{\theta \theta^{\prime}}$ should avoid the trace $\delta_{1}=\delta_{1}\left(\theta, \theta^{\prime}\right)$ of $\Sigma\left(\mu_{1} ; \theta\right)$ in the $\zeta$-plane which (in view of formula (40)) is described by the following formula :

$$
\delta_{1}=\left\{\zeta \in \mathbb{C} ; \zeta \sin \theta \sin \theta^{\prime}+\cos \theta \cos \theta^{\prime}-\rho=0 ; \rho \geq \rho_{1},\left(\rho_{1} \geq 1\right)\right\}
$$

For $\theta$ or $\theta^{\prime} \notin \pi \mathbb{Z}, \delta_{1}$ is a half-line, also conveniently represented by the following two (equivalent) formulae :

$$
\begin{array}{ll}
\zeta-1=\frac{-\cos \left(\theta-\theta^{\prime}\right)+\rho}{\sin \theta \sin \theta^{\prime}}, & \rho \geq \rho_{1}, \\
\zeta+1=\frac{-\cos \left(\theta+\theta^{\prime}\right)+\rho}{\sin \theta \sin \theta^{\prime}}, & \rho \geq \rho_{1} .
\end{array}
$$

We will also denote by $\boldsymbol{\delta}_{\theta \theta^{\prime}}$ the support of $\boldsymbol{\delta}_{1}\left(\theta, \theta^{\prime}\right)$, namely

$$
\delta_{\theta \theta^{\prime}}=\left\{\zeta \in \mathbb{C} ; \zeta=\frac{-\cos \theta \cos \theta^{\prime}+\rho}{\sin \theta \sin \theta^{\prime}} ; \rho \in \mathbb{R}\right\}
$$

i) The initial situation : the choice of $\Gamma_{\theta}$ for $\theta$ real.

From Eqs. (46), (47), it readily follows that for $\theta$ and $\theta^{\prime}$ real, with $\theta$ and $\theta^{\prime} \notin \pi \mathbb{Z}, \delta_{1}$ is contained in $]-\infty,-1[\cup]+1,+\infty\left[\left(\delta_{1}\right.\right.$ being empty, in view of Eq. (45), for $\theta$ or $\theta^{\prime} \in \pi \mathbb{Z}$ ).

$$
\text { TOME } 120-1992-\mathrm{N}^{\circ} 2
$$


The previous requirements a) and b) will then be fulfilled if the chosen cycle $\Gamma_{\theta}$ is the sphere $S$ (equipped with its natural orientation), represented in the space of variables $\theta^{\prime}, \zeta$ and $\vec{\alpha}^{\prime}$ by the following sets

$$
\gamma_{\theta}=\mathbb{R} / 2 \pi \mathbb{Z} \equiv[-\pi,+\pi], \quad \zeta \in[-1,+1], \quad \vec{\alpha}^{\prime} \in \mathbb{S}_{d-3},
$$

with $\left(\theta^{\prime}, \zeta, \vec{\alpha}^{\prime}\right) \equiv\left(\theta^{\prime}, \zeta,-\vec{\alpha}^{\prime}\right)$ (in view of $(R)$ : see Eqs. $(20)$ ). With this specification of $\Gamma_{\theta}$ for $\theta$ real, the integral (41) is equal to $\mathcal{K}_{\mid S_{0} \times S} * \mathcal{F}_{\mid S}$ so that property a) of THEOREM 1 is "built in" in our definition.

ii) The continuous distortion of $\Gamma_{\theta}$ for $\theta$ varying in the set : $\{\theta=$ $u+i v \in \mathbb{C} ; u \neq 0, u \neq \pi\}$.

The distortion that we define will take place in the space $\mathbb{C}_{\left(\theta^{\prime}, \zeta\right)}^{2}$ (i.e. will concern $\gamma_{\theta}$ and $\left.\mathbf{L}_{\theta \theta^{\prime}}\right)$, but will not affect the real cycle $\left\{\vec{\alpha}^{\prime} \in \mathbb{S}_{d-3}\right\}$ which will be kept fixed for all values of $\theta$ in $\mathcal{J}_{\mu}$. The fact that the cuts $\Xi(\mu)$ and $\Sigma\left(\mu_{1}, \theta\right)$ are $\mathrm{SO}(d-2)$-invariant (i.e. that formulae (39), (40), do not involve the variable $\vec{\alpha}^{\prime}$ ) implies that our construction is the same for all dimensions $d(d \geq 3)$.

We first investigate whether the half-line $\delta_{1}$ may contain the points $\zeta=1$ or -1 . In view of formulae (46) and (47), this situation occurs in the following subset of $\mathbb{C}_{\theta^{\prime}} / 2 \pi \mathbb{Z}$ :

$$
\delta_{1}=\left\{\theta^{\prime}=\theta+i v^{\prime} \text { or } \theta^{\prime}=-\theta+i v^{\prime}, \text { with }\left|v^{\prime}\right| \geq \mu_{1}\right\}
$$

Since such a situation must be avoided (in view of the fact that $\zeta=1$ and $\zeta=-1$ are the end-points of $\mathbf{L}_{\theta \theta^{\prime}}$ in the specification of $\left.i\right)$ ), we are led to define a continuous distortion of $\gamma_{\theta}$ such that for each $\theta, \gamma_{\theta}$ avoids the set $\delta_{1} \cup \delta_{2}$.

The construction of $\Gamma_{\theta}$ will also be simplified thanks to the following symmetry properties which are immediately implied by Eq. (45) :

$$
\begin{aligned}
\left(S_{1}\right) \quad \forall\left(\theta, \theta^{\prime}\right), \delta_{1}\left(\bar{\theta}, \bar{\theta}^{\prime}\right)=\delta_{1}\left(-\bar{\theta},-\bar{\theta}^{\prime}\right)=\delta_{1}\left(\pi-\bar{\theta}, \pi-\bar{\theta}^{\prime}\right)=\overline{\delta_{1}\left(\theta, \theta^{\prime}\right)} \\
\left(S_{2}\right) \quad \forall\left(\theta, \theta^{\prime}\right), \text { the sets } \delta_{1}\left(\theta,-\theta^{\prime}\right) \text { and } \delta_{1}\left(\theta, \theta^{\prime}\right) \text { are symmetric with } \\
\\
\text { respect to the origin in the } \zeta \text {-plane. }
\end{aligned}
$$

Property $\left(S_{1}\right)$ suggests that it might be sufficient to define an appropriate distortion of $\Gamma_{\theta}$ for $\theta$ varying in the subset

$$
\dot{\mathcal{J}}^{(0)}=\left\{\theta=u+i v \in \mathbb{C} ; 0<u \leq \frac{1}{2} \pi, v \geq 0\right\}
$$

of $\dot{\mathcal{J}}_{\mu}$ and to complete the definition of this distortion in the rest of $\dot{\mathcal{J}}_{\mu}$ by using the relevant symmetries. 
Property $\left(S_{2}\right)$ suggests that one should first define the part $\gamma_{\theta}^{+}$contained in the strip $0 \leq \operatorname{Re} \theta^{\prime} \leq \pi$ and the corresponding part

$$
\Gamma_{\theta}^{+}=\left\{\left(\theta^{\prime}, \varphi^{\prime}, \vec{\alpha}^{\prime}\right) ; \theta^{\prime} \in \gamma_{\theta}^{+}, \varphi^{\prime} \in \operatorname{supp} L_{\theta \theta^{\prime}}, \vec{\alpha}^{\prime} \in \mathbb{S}_{d-3}\right\} / R
$$

of $\operatorname{supp} \Gamma_{\theta}$; then, the complement

$$
\Gamma_{\theta}^{-}=\left\{\left(\theta^{\prime}, \varphi^{\prime}, \vec{\alpha}^{\prime}\right) ; \theta^{\prime} \in \gamma_{\theta}^{-}, \varphi^{\prime} \in \operatorname{supp} L_{\theta \theta^{\prime}}, \vec{\alpha}^{\prime} \in \mathbb{S}_{d-3}\right\} / R
$$

should be defined by the following prescription :

$$
\begin{aligned}
\gamma_{\theta}^{-} & =\left\{\theta^{\prime} ;-\theta^{\prime} \in \gamma_{\theta}^{+}\right\} \text {and } \\
\operatorname{supp} \mathbf{L}_{\theta \theta^{\prime}} & =\left\{\zeta \in \mathbb{C} ;-\zeta \in \operatorname{supp} \mathbf{L}_{\theta\left(-\theta^{\prime}\right)}\right\} .
\end{aligned}
$$

For each $\theta$ in the set $\dot{J}^{(0)}$, we adopt the following choice for $\gamma_{\theta}$ which satisfies in an obvious way our previous requirement, namely supp $\gamma_{\theta} \cap\left(\delta_{1} \cup \delta_{2}\right)=\phi$. We define $\gamma_{\theta}^{+}$as the broken line consisting in the three segments $[0, \theta],[\theta, 2 u]$ and $[2 u, \pi]$ and we put supp $\gamma_{\theta}=\gamma_{\theta}^{+} \cup \gamma_{\theta}^{-}$ (see Figures 3.a) and 3.b)). For $u=\operatorname{Re} \theta$ fixed and $v=\operatorname{Im} \theta$ increasing from the value $v=0$, the path $\gamma_{\theta}$ thus defined is continuously distorted from its initial situation (i.e. $[-\pi,+\pi]$ ) by dilatations parallel to the $v^{\prime}$-axis and proportional to $v$.

We now have to implement our construction of $\Gamma_{\theta}^{+}$by specifying the path $\mathbf{L}_{\theta \theta^{\prime}}$ in the $\zeta$-plane, for all values of $\theta^{\prime}$ in $\gamma_{\theta}^{+}$. The following property will be useful.

Auxiliary Lemma. - Let $m_{\theta \theta^{\prime}}=\boldsymbol{\delta}_{\left(\theta \theta^{\prime}\right)} \cap\{\zeta ; \zeta \in \mathbb{R}\}$. Then, if $\theta^{\prime}$ varies in the strip $\Theta_{v}=\left\{\theta^{\prime}=u^{\prime}+i v^{\prime} ;\left|v^{\prime}\right|<|v|(v=\operatorname{Im} \theta)\right\}$, the subset of points $\theta^{\prime}$ for which $m_{\theta \theta^{\prime}}$ is a point between -1 and +1 , is composed of two rectangles, namely:

$$
\begin{aligned}
& \left\{\theta^{\prime}=u^{\prime}+i v^{\prime} ;\left|v^{\prime}\right|<|v|, \quad\left|u^{\prime}-\frac{1}{2} \pi\right|<\left|u-\frac{1}{2} \pi\right|\right. \\
& \left.\qquad \text { or }\left|u^{\prime}+\frac{1}{2} \pi\right|<\left|u-\frac{1}{2} \pi\right| \quad(\bmod 2 \pi)\right\}
\end{aligned}
$$

if $0<u<\pi$, or

$$
\begin{aligned}
\left\{\theta^{\prime}=u^{\prime}+i v^{\prime} ;\left|v^{\prime}\right|<|v|,\right. & \left|u^{\prime}-\frac{1}{2} \pi\right|<\left|u+\frac{1}{2} \pi\right| \\
& \text { or } \left.\left|u^{\prime}+\frac{1}{2} \pi\right|<\left|u+\frac{1}{2} \pi\right| \quad(\bmod 2 \pi)\right\}
\end{aligned}
$$

if $-\pi<u<0$.

(Note that these rectangles correspond to the hatchings on Figures 3.a and 3.b.)

$$
\text { TOME } 120-1992-\mathrm{N}^{\circ} 2
$$




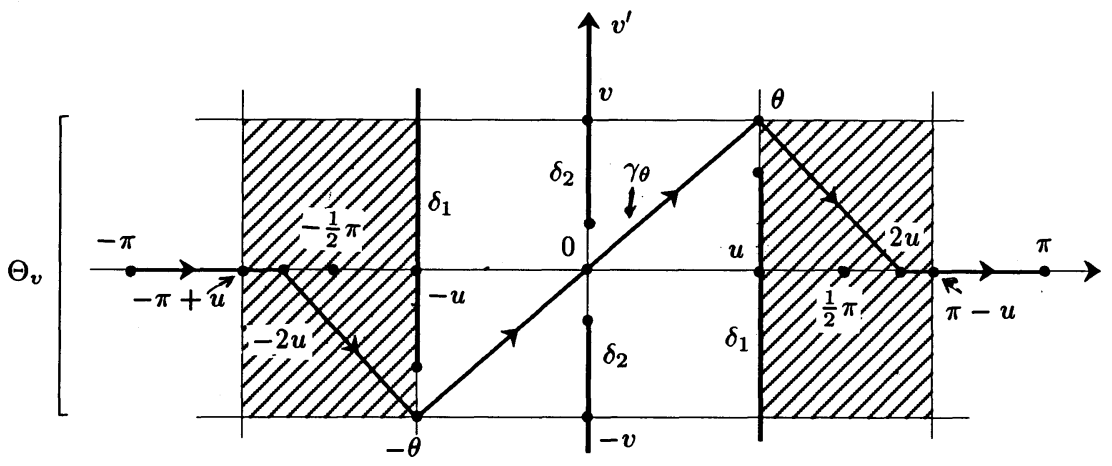

Figure 3.a $: \operatorname{Re} \theta<\frac{1}{3} \pi$.

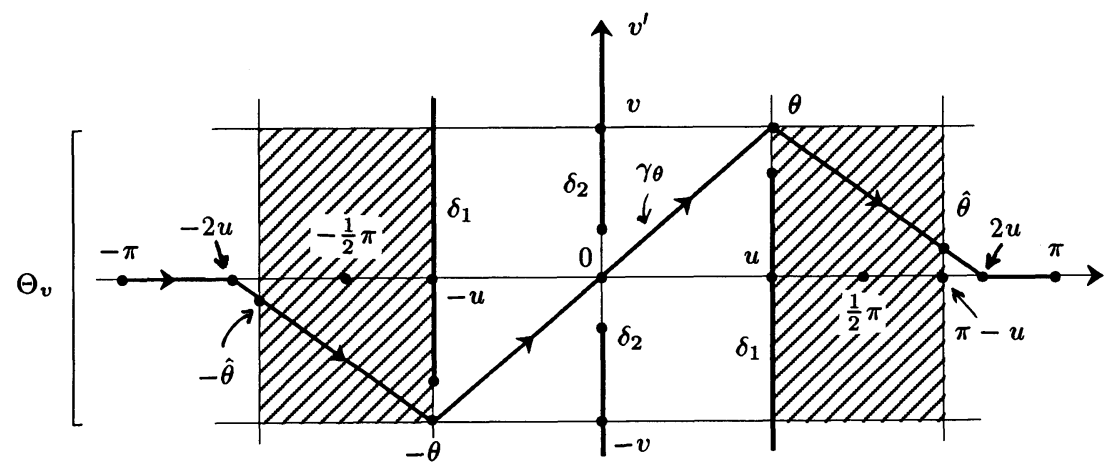

Figure 3.b : $\frac{1}{3} \pi<\operatorname{Re} \theta<\frac{1}{2} \pi$.

Figure 3. The path $\gamma_{\theta}$ in the $\theta^{\prime}$-plane.

Proof. - In view of formula (48), the abscissa $\zeta\left(m_{\theta \theta^{\prime}}\right)$ of $m_{\theta \theta^{\prime}}$ must be a real solution of the equation : $\zeta\left(m_{\theta \theta^{\prime}}\right) \times \sin \theta \sin \theta^{\prime}=-\cos \theta \cos \theta^{\prime}+\rho$ ( $\rho$ real). Therefore we have :

$$
\zeta\left(m_{\theta \theta^{\prime}}\right)=-\frac{\operatorname{Im}\left(\cos \theta \cos \theta^{\prime}\right)}{\operatorname{Im}\left(\sin \theta \sin \theta^{\prime}\right)}
$$

The condition $\left|\zeta\left(m_{\theta \theta^{\prime}}\right)\right| \leq 1$ is then equivalent to

$$
\operatorname{Im} \cos \left(\theta+\theta^{\prime}\right) \operatorname{Im} \cos \left(\theta-\theta^{\prime}\right) \leq 0
$$




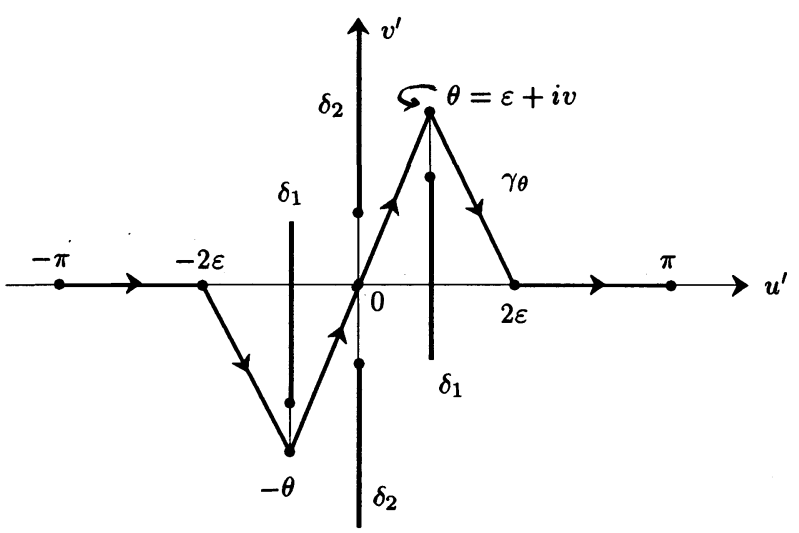

Figure 3.c : $\theta=\varepsilon+i v$.

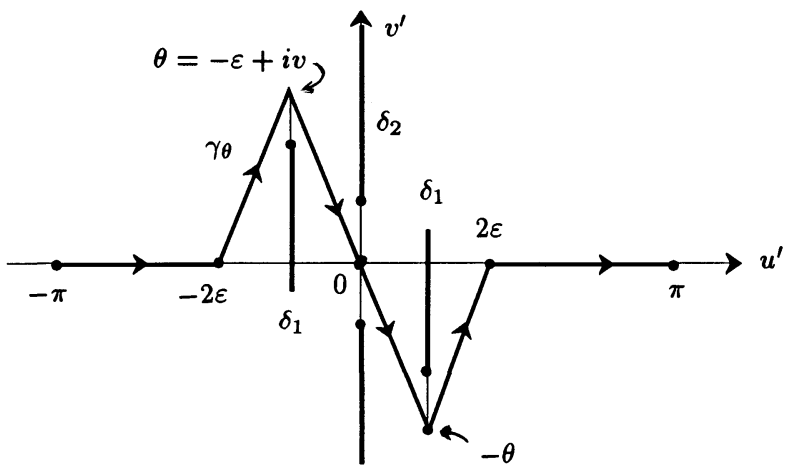

Figure 3.d : $\theta=-\varepsilon+i v$.

Figure 3. The path $\gamma_{\theta}$ in the $\theta^{\prime}$-plane.

which can be rewritten :

$$
\sin \left(u+u^{\prime}\right) \sin \left(u-u^{\prime}\right) \sinh \left(v+v^{\prime}\right) \sinh \left(v-v^{\prime}\right) \leq 0 .
$$

For $\theta^{\prime}$ in the strip $\Theta_{v}$, one has $\left|v^{\prime}\right|<|v|$, which implies

$$
\sinh \left(v+v^{\prime}\right) \sinh \left(v-v^{\prime}\right)>0
$$

we are thus led to the condition :

$$
\sin \left(u+u^{\prime}\right) \sin \left(u-u^{\prime}\right) \leq 0
$$

which yields the regions described in the auxiliary lemma.

TOME $120-1992-\mathrm{N}^{\circ} 2$ 
Remark. - The set of points $\theta^{\prime}$ such that $m_{\theta \theta^{\prime}}$ is the real $\zeta$-axis is composed of the corners $u-i v, \pi-u+i v,-u+i v, u-\pi-i v$ of the previous rectangular regions.

\section{Consequences}

$\alpha$ ) When $\theta^{\prime}$ varies in the complement in $\Theta_{v}$ of the union of rectangular regions described in the auxiliary lemma, the point $m_{\theta \theta^{\prime}}$ is outside $[-1,+1]$; this is the case in particular when $\theta^{\prime}$ belongs to the linear segment $[0, \theta]$ and to the $\operatorname{arc}[\hat{\theta}, \pi]$ of $\gamma_{\theta}^{+}$, where $\hat{\theta}=\pi-u$ if $u=\operatorname{Re} \theta \leq \frac{1}{3} \pi$ and $\hat{\theta}=\pi-u+i \hat{v}$, with $\hat{\theta} \in[\theta, 2 u]$ if $\frac{1}{3} \pi \leq u \leq \frac{1}{2} \pi$. For all these values of $\theta^{\prime}$, the path $\mathbf{L}_{\theta \theta^{\prime}}$ can thus be kept fixed and equal to $[-1,+1]$ (see Figure 3.a), 3.b) and 4.a)). Note that in order to yield the natural orientation of the sphere $S$ in the initial situation, this path is oriented from +1 to -1 (since it is the image of the path $\varphi^{\prime}=i \psi^{\prime}, 0 \leq \psi^{\prime} \leq \pi$, oriented from 0 to $i \pi$ ).

$\beta$ ) When $\theta^{\prime}$ belongs to the remaining part $\hat{\gamma}_{\theta}^{+}$of $\gamma_{\theta}^{+}$(i.e. the arc of $\gamma_{\theta}^{+}$ with end-points $\theta, \hat{\theta}$ which is contained in the rectangular regions of the auxiliary lemma), the situation in the $\zeta$-plane may necessitate a distortion of $\mathbf{L}_{\theta \theta^{\prime}}$, since $m_{\theta \theta^{\prime}}$ is a point between -1 and +1 .

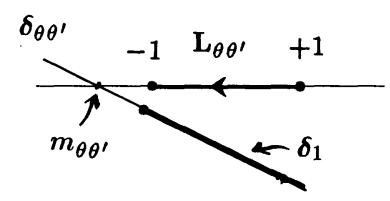

Figure 4.a : $\theta^{\prime} \in[\pi-u, \pi]$.

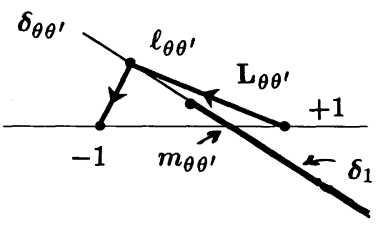

Figure 4.b : $\theta^{\prime} \in \hat{\gamma}_{\theta}^{+}$

Figure 4. The path $\mathbf{L}_{\theta \theta^{\prime}}$ in the $\zeta$-plane.

We indicate the following way of implementing the continuous distortion of $\mathbf{L}_{\theta \theta^{\prime}}$ when $\theta^{\prime}$ varies on $\hat{\gamma}_{\theta}^{+}$; its justification will be given just below. We define $\mathbf{L}_{\theta \theta^{\prime}}$ as the broken path $\left[-1, \ell_{\theta \theta^{\prime}}\right] \cup\left[\ell_{\theta \theta^{\prime}}+1\right]$ oriented from +1 to -1 (see Figure $4 . \mathrm{b})$ ), where the point $\ell_{\theta \theta^{\prime}}$ is introduced by the following formula :

$$
\ell_{\theta \theta^{\prime}}=\frac{-\cos \theta \cos \theta^{\prime}+\rho\left(\theta, \theta^{\prime}\right)}{\sin \theta \sin \theta^{\prime}}
$$

with

$$
\rho\left(\theta, \theta^{\prime}\right)=\frac{\mathrm{d}\left(\theta^{\prime}, \hat{\theta}\right)}{\mathrm{d}(\theta, \hat{\theta})}\left[\cosh \left(v+v^{\prime}\right)+1\right]-\cosh \left(v+v^{\prime}\right)
$$


in the latter, $\mathrm{d}\left(\theta^{\prime}, \hat{\theta}\right)$ denotes the distance from $\theta^{\prime}$ to $\hat{\theta}$ taken along the path $\hat{\gamma}_{\theta}^{+}$. It is clear that at the end-points $\theta^{\prime}=\theta$ and $\theta^{\prime}=\hat{\theta}$ of the latter, formula (49), (50) yield $\ell_{\theta \theta}=1$ and $\ell_{\theta \hat{\theta}}=-1$, so that $\mathbf{L}_{\theta \theta^{\prime}}$ tends to $[-1,+1]$ at both ends (see Figure 4.c, 4.d)). Let us now check that :

$$
\forall \theta^{\prime} \in \hat{\gamma}_{\theta}^{+}, \quad \operatorname{supp} \mathbf{L}_{\theta \theta^{\prime}} \cap \delta_{1}\left(\theta, \theta^{\prime}\right)=\phi .
$$

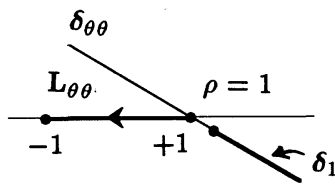

Figure 4.c : $\theta^{\prime}=\theta$.

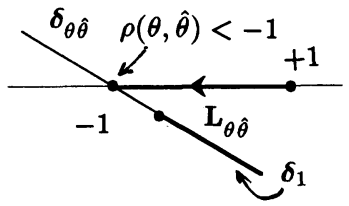

Figure 4.d : $\theta^{\prime}=\hat{\theta}$.

Figure 4. The path $\mathbf{L}_{\theta \theta^{\prime}}$ in the $\zeta$-plane.

The point $\ell_{\theta \theta^{\prime}}$ has been chosen so as to satisfy Eq. (48) for the value $\rho=\rho\left(\theta, \theta^{\prime}\right)$, and since the latter varies between $-\cosh (v+\hat{v})$ and 1 when $\theta^{\prime}$ moves on $\gamma_{\theta}^{+}$(see Eq. (50)), one concludes that :

$$
\forall \theta^{\prime} \in \hat{\gamma}_{\theta}^{+}, \quad \ell_{\theta \theta^{\prime}} \in \delta_{\theta \theta^{\prime}} \text { and } \ell_{\theta \theta^{\prime}} \notin \delta_{1}\left(\theta, \theta^{\prime}\right)
$$

Therefore (since $\boldsymbol{\delta}_{\theta \theta^{\prime}}$ does not contain the points $\zeta= \pm 1$ ), the property expressed by formula (51) is valid (see Figure 4.b)).

Finally we notice that completing the definition of $\Gamma_{\theta}$ by $\operatorname{supp} \Gamma_{\theta}=$ $\Gamma_{\theta}^{+} \cup \Gamma_{\theta}^{-}$(according to the previous prescription suggested by $\left(S_{2}\right)$ ) is consistent with the continuity requirement, since $\mathbf{L}_{\theta 0}$ and $\mathbf{L}_{\theta \pi}$ coincide with $[-1,+1]$ (Note that the orientation on $\Gamma_{\theta}$ is indicated in an obvious way by the initial situation, when $\theta$ is real).

So far, we have constructed the cycle $\Gamma_{\theta}$ for $\theta$ varying in the subset $\dot{J}^{(0)}$ of $\dot{\mathcal{J}}_{\mu}$. This construction defines a section $\theta \rightarrow \dot{\Gamma}_{\theta}$ of a homology bundle with basis $\dot{\mathcal{J}}^{(0)}$ associated with the analyticity domain $\bigcup_{\theta \in \dot{J}^{(0)}}(\theta, D(\theta))$ of the integrand of Eq. (41).

It follows (see e.g. [7]) that the function $\mathcal{G}(z(\theta)$ ) defined by the integral (41) is an analytic function of $\theta$ in the set $\dot{J}^{(0)}$. In order to establish the analyticity of $\mathcal{G}(z(\theta))$ in the domain $\dot{\mathcal{J}}_{\mu}$, we proceed with the following steps :

$$
\text { TOME } 120-1992-\mathrm{N}^{\circ} 2
$$


1) By taking into account the relation $\delta_{1}\left(\bar{\theta}, \bar{\theta}^{\prime}\right)=\overline{\delta_{1}\left(\theta, \theta^{\prime}\right)}$ of $\left(S_{1}\right)$, we can define $\Gamma_{\theta}$ for $\theta$ in the set $\left\{\theta \in \mathbb{C} ; 0<u \leq \frac{1}{2} \pi, v \leq 0\right\}$ by the prescription : $\gamma_{\theta}=\overline{\gamma_{\bar{\theta}}}$ (with $\bar{\theta} \in \dot{\mathcal{J}}^{(0)}$ ) and $\mathbf{L}_{\theta \theta^{\prime}}=\overline{\mathbf{L}_{\bar{\theta} \bar{\theta}^{\prime}}}$. The continuity requirement between this new set and $\dot{J}^{(0)}$ is fulfilled in an obvious way at $v=0$ (with $\theta^{\prime} \in[-\pi,+\pi], \zeta \in[-1,+1]$ ).

2) By taking into account the relation $\delta_{1}\left(\pi-\bar{\theta}, \pi-\bar{\theta}^{\prime}\right)=\overline{\delta_{1}\left(\theta, \theta^{\prime}\right)}$ of $\left(S_{1}\right)$, we can define $\Gamma_{\theta}^{+}$for $\theta$ in the strip $\left\{\theta \in \mathbb{C} ; \frac{1}{2} \pi \leq u<\pi\right\}$ by the prescription : $\gamma_{\theta}^{+}=\left\{\theta^{\prime} ; \pi-\bar{\theta}^{\prime} \in \gamma_{\pi-\bar{\theta}}^{+}\right\}, \mathbf{L}_{\theta, \theta^{\prime}}=\overline{\mathbf{L}_{\pi-\bar{\theta}, \pi-\bar{\theta}^{\prime}}}$, and complete again the definition of $\operatorname{supp} \Gamma_{\theta}=\Gamma_{\theta}^{+} \cup \Gamma_{\theta}^{-}$as above (according to $\left.\left(S_{2}\right)\right)$. The continuity requirement between the strips $0<u \leq \frac{1}{2} \pi$ and $\frac{1}{2} \pi \leq u<\pi$ is fulfilled since $\gamma_{\pi / 2+i v}^{+}$is symmetric by itself with respect to the line $u=\frac{1}{2} \pi$ and since the corresponding path $\mathbf{L}_{\pi / 2+i v, \theta^{\prime}}$ is (for all $\theta^{\prime}$ ) equal to $[-1,+1]$.

3) By taking into account the last relation $\delta_{1}\left(-\bar{\theta},-\bar{\theta}^{\prime}\right)=\overline{\delta_{1}\left(\theta, \theta^{\prime}\right)}$, we can define $\Gamma_{\theta}^{+}$for $\bar{\theta}$ in the whole strip $\{\theta \in \mathbb{C} ;-\pi<u<0\}$ by the prescription $\gamma_{\theta}^{+}=\left\{\theta^{\prime} ;-\bar{\theta}^{\prime} \in \gamma_{-\bar{\theta}}^{+}\right\}, \mathbf{L}_{\theta \theta^{\prime}}=\overline{\mathbf{L}_{-\bar{\theta},-\bar{\theta}^{\prime}}}$ and complete again $\operatorname{supp} \Gamma_{\theta}$ as above (according to $\left(S_{2}\right)$ ).

4) The continuity requirement for $\Gamma_{\theta}$ is not satisfied across the lines $u=0$ and $u= \pm \pi$, except for $v=0$ (i.e. at the points $\theta=0$ or $\theta= \pm \pi)$, since $\gamma_{0}=\gamma_{ \pm \pi}=[-\pi,+\pi]$ and $\mathbf{L}_{0 \theta^{\prime}}=\mathbf{L}_{ \pm \pi, \theta^{\prime}}=[-1,+1]$. This is however sufficient for our homology section $\theta \rightarrow \dot{\Gamma}_{\theta}$, now defined in the set $\{\theta \in \mathbb{C} ; u \neq 0, u \neq \pi\} \cup\{0\} \cup\{\pi\}$, to provide the analytic continuation of the function $\mathcal{G}(z(\theta)$ ) (via Eq. (41)) in an open neighbourhood of the previous set. As a matter of fact, one shows directly (by using the real cycle $\left.\theta^{\prime} \in[-\pi,+\pi], \zeta \in[-1,+1]\right)$ that $\mathcal{G}(z(\theta))$ is actually analytic at least on the intervals $\left\{\theta=i v ;|v|<\mu_{1}\right\}$ and $\left\{\theta=\pi+i v ;|v|<\mu_{1}\right\}$.

A more complete analysis of the situations $\theta=i v$ and $\theta=\pi+i v$ will be given below in part B). It will include :

- The proof of the fact that $\mathcal{G}(z(\theta))$ is analytic for $\theta=i v$, with $|v|<\mu=\mu_{1}+\mu_{2}$, and for $\theta=\pi+i v, v \in \mathbb{R}$, which will complete the first part of LEMma 1 stating the analyticity of $\mathcal{G}(z)$ in $D_{\mu, 0}$.

- The proof of the discontinuity formula $(36)$ for $\mathcal{G}(z(\theta))$ across the cut $\theta=i v, v \geq \mu$.

\section{B) Computing the discontinuity $\Delta_{+} \mathcal{G}$}

The situation where $u$ tends to 0 or to $( \pm) \pi$ will necessitate a passage to the limit for the cycle $\Gamma_{\theta}$ which, in particular, will generate the boundary set (or "cut") $X_{\mu, 0}^{+}$for the domain of the function $\mathcal{G}(z)$ defined by Eq. (41).

We shall first consider the case when $u$ tends to zero, and give the 
detailed derivation of formula (36) for the discontinuity $\Delta_{+} \mathcal{G}(z(i v))$ across the half-line $\theta=i v, v \geq 0$, namely :

$$
\Delta_{+} \mathcal{G}(z(i v))=i \lim _{\substack{\varepsilon \rightarrow 0 \\ \varepsilon>0}}[\mathcal{G}(z(\varepsilon+i v))-\mathcal{G}(z(-\varepsilon+i v))]
$$

We make use of the previous construction of $\Gamma_{\theta}$ (see A)) in the corresponding limiting situations $\operatorname{Re} \theta= \pm \varepsilon \rightarrow 0$. These situations in the $\theta^{\prime}$-plane are represented respectively on Figures 3.c) and 3.d).

Each term at the r.h.s. of Eq. (52) is expressed by an integral of the form (41) which contains :

i) a contribution coming from the horizontal parts of $\gamma_{\theta}$, namely $[-\pi,-2 \varepsilon]$ and $[2 \varepsilon, \pi]$

ii) contributions coming from the broken lines of $\gamma_{\theta}$ neighbouring the line $u^{\prime}=0$, namely; $[-2 \varepsilon,-\varepsilon-i v, 0, \varepsilon+i v, 2 \varepsilon]$ for the case $\theta=\varepsilon+i v$ (see Figure 3.c)), and $[-2 \varepsilon,-\varepsilon+i v, 0, \varepsilon-i v, 2 \varepsilon]$ for the case $\theta=-\varepsilon+i v$ (see Figure 3.d)).

We first show that the contributions to the r.h.s. of Eq. (52) described in i) cancel in the limit $\varepsilon \rightarrow 0$. As a matter of fact in both contributions, the same function $\mathcal{K}\left(z(\theta), z^{\prime}\left(\theta^{\prime}, \varphi^{\prime}, \vec{\alpha}^{\prime}\right)\right) \mathcal{F}\left(z^{\prime}\left(\theta^{\prime}, \varphi^{\prime}, \vec{\alpha}^{\prime}\right)\right)$ is integrated on the same set

$$
\left\{\left(\theta^{\prime}, \varphi^{\prime}, \vec{\alpha}^{\prime}\right) ; \theta^{\prime} \in[-\pi, 0] \cup[0, \pi], \varphi^{\prime} \in \operatorname{supp} L_{\theta \theta^{\prime}}, \vec{\alpha}^{\prime} \in \mathbb{S}_{d-3}\right\}
$$

where $\operatorname{supp} L_{\theta \theta^{\prime}}$ is defined by its image $\operatorname{supp} \mathbf{L}_{\theta \theta^{\prime}}=\left[-1, \ell_{\theta \theta^{\prime}}\right] \cup\left[\ell_{\theta \theta^{\prime}},+1\right]$. In fact, for $\theta=\lim _{\varepsilon \rightarrow 0}(\varepsilon+i v)$ and $\theta^{\prime} \in[0, \pi]$, the point $\ell_{\theta \theta^{\prime}}$ is defined by Eq. (49) which yields :

$$
\ell_{\theta \theta^{\prime}}=\frac{-\cosh v \cos \theta^{\prime}+\rho\left(i v, \theta^{\prime}\right)}{i \sinh v \sin \theta^{\prime}} \in i \mathbb{R} .
$$

We then check that for $\theta=\lim _{\varepsilon \rightarrow 0}(-\varepsilon+i v)$, the relevant definition of $L_{\theta \theta^{\prime}}$ (using the symmetries $\left(S_{1}\right)$ and $\left(S_{2}\right)$, as described in A)) coincides with the previous one, since in this situation $\ell_{\theta \theta^{\prime}}=-\overline{\ell_{\theta \theta^{\prime}}}$. A similar check holds for $\theta^{\prime} \in[-\pi, 0]$.

We now analyse the contributions to the r.h.s. of Eq. (52) described in ii) and show that they produce in the limit $\varepsilon \rightarrow 0$ the expression $\Delta_{+} \mathcal{K} \diamond \Delta_{+} \mathcal{F}$ of the discontinuity $\Delta_{+} \mathcal{G}$ of $\mathcal{G}$.

We shall first describe the contribution coming from the upper broken line $[0, \varepsilon+i v, 2 \varepsilon]$ in the first term at the r.h.s. of Eq. (52). Since $\operatorname{Re} \theta^{\prime}$ is

$$
\text { TOME } 120-1992-\mathrm{N}^{\circ} 2
$$




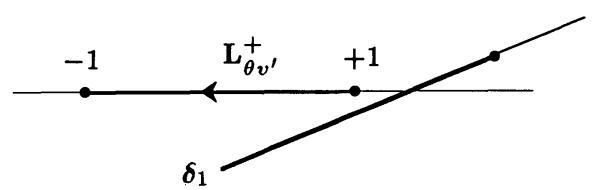

Figure 4.e : $\theta=\varepsilon+i v, \theta^{\prime} \in[0, \theta]$.

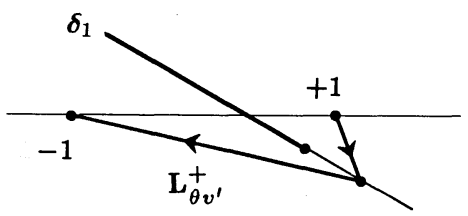

Figure 4.g : $\theta=-\varepsilon+i v, \theta^{\prime} \in[-2 \varepsilon, \theta] . \quad$ Figure 4.h : $\theta=-\varepsilon+i v, \theta^{\prime} \in[\theta, 0]$.

Figure 4. The path $\mathbf{L}_{\theta \theta^{\prime}}$ in the $\zeta$-plane.

positive on this path (and $\operatorname{Re} \theta^{\prime} \rightarrow 0$ with $\varepsilon \rightarrow 0$ ), the relevant limit of $\mathcal{F}\left(z^{\prime}\left(\theta^{\prime}, \varphi^{\prime}, \vec{\alpha}^{\prime}\right)\right)$ in the corresponding integrand of Eq. (41) will be $\mathcal{F}_{+}$(in view of Eq. (33)).

Now, by taking into account the definition of $\mathbf{L}_{\theta \theta^{\prime}}$ given in $\mathrm{A}$ ), we see that :

a) For $\theta^{\prime} \in[0, \varepsilon+i v]$ with $\operatorname{Im} \theta^{\prime}=v^{\prime}, \mathbf{L}_{\theta \theta^{\prime}}$ is the set $[-1,+1]$ oriented from +1 to -1 , denoted here by $\mathbf{L}_{\theta v^{\prime}}^{+}$, and the situation in the $\zeta$-plane is the one represented on Figure 4.e).

b) For $\theta^{\prime} \in[\varepsilon+i v, 2 \varepsilon]$ with $\operatorname{Im} \theta^{\prime}=v^{\prime}, \mathbf{L}_{\theta \theta^{\prime}}$ must be defined as the distorted path $\mathbf{L}_{\theta v^{\prime}}^{-}=\left[-1, \ell_{\theta \theta^{\prime}}\right] \cup\left[\ell_{\theta \theta^{\prime}},+1\right]$ oriented from +1 to -1 (with $\ell_{\theta \theta^{\prime}}$ given for example by Eqs. (49), (50)), as indicated by Figure 4.f).

The limit of the situations pictured in Figures 4.e) and 4.f) for $\varepsilon$ tending to zero yields (for the same limiting value $\theta^{\prime}=i v^{\prime} ; 0<v^{\prime}<v$ ) the situation illustrated on Figure 5.a), after an appropriate distortion of the path $\mathbf{L}_{\theta v^{\prime}}^{-}$. Figure 5.b) exhibits a clockwise contour $\mathbf{L}$ which is homologous to $\mathbf{L}_{\theta v^{\prime}}^{+}-\mathbf{L}_{\theta v^{\prime}}^{-}$(in the domain of $\mathcal{K}$ ) and which encloses the linear segment $\left[1, \zeta_{1}\right], \zeta_{1}$ being the origin of the cut $\delta_{1}$, given (in view of Eq. (46)) by the 
following formula :

$$
\zeta_{1}-1=\cosh \varphi_{1}^{\prime}\left(v, v^{\prime}\right)-1=\frac{\cosh \left(v-v^{\prime}\right)-\cosh \mu_{1}}{\sinh v \sinh v^{\prime}}
$$

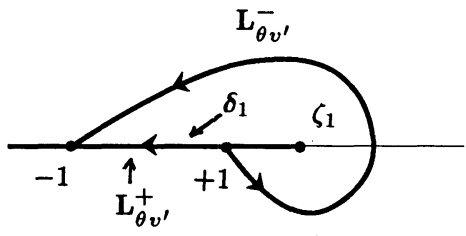

Figure 5.a.

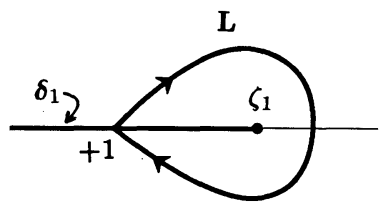

Figure 5.b.

Figure 5. The $\zeta$-plane : limiting situation for $z=z(\theta)$ (from $\operatorname{Re} \theta=\varepsilon>0$ ) in the cut $X_{\mu, 0}$.

The corresponding contribution from the integral (41) to the r.h.s. of Eq. (52) can therefore be written as follows :

$$
\begin{aligned}
(54)-\int \omega\left(\vec{\alpha}^{\prime}\right) \int_{0}^{v}\left(\sinh v^{\prime}\right)^{d-2} \mathrm{~d} v^{\prime} \\
\\
\int_{L} \mathcal{K}\left[z(i v), z^{\prime}\left(i v^{\prime}, \varphi^{\prime}, \vec{\alpha}^{\prime}\right)\right] \mathcal{F}_{+}\left[z^{\prime}\left(i v^{\prime}, \varphi^{\prime}, \alpha^{\prime}\right)\right]\left(\sinh \varphi^{\prime}\right)^{d-3} \mathrm{~d} \varphi^{\prime}
\end{aligned}
$$

where the clockwise path $L$ enclosing the cut $\left\{\varphi^{\prime} \in\left[0, \varphi_{1}^{\prime}\right]\right\}$ in the $\varphi^{\prime}$-plane (see Figure 6)) is the inverse image of the path $\mathbf{L}$ of the $\zeta$-plane.

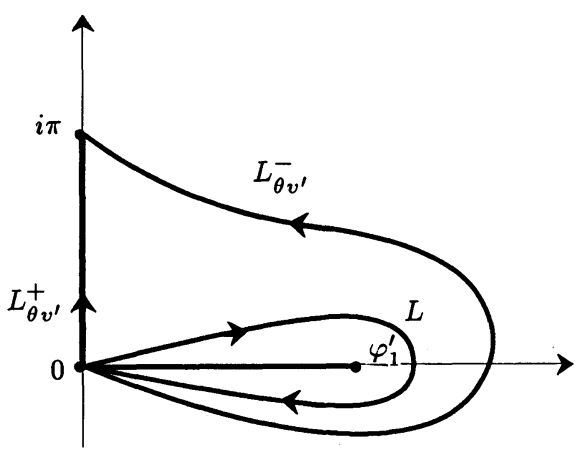

Figure 6. The $\varphi^{\prime}$-plane. 
By using the parametrization (П), one then checks that the relevant limits of $\mathcal{K}\left[z(i v), z^{\prime}\left(i v^{\prime}, \varphi^{\prime}, \vec{\alpha}^{\prime}\right)\right]$ on the cut $\left[0, \varphi_{1}^{\prime}\left(v, v^{\prime}\right)\right]$ from the sides $\operatorname{Im} \varphi^{\prime}>0$ and $\operatorname{Im} \varphi^{\prime}<0$ (or $\operatorname{Im} \zeta>0$ and $\operatorname{Im} \zeta<0$ ) are respectively the boundary values $\mathcal{K}_{+}$and $\mathcal{K}_{-}$defined at the end of section 2 (since $\left.\operatorname{Im}\left[\left(z-z^{\prime}\right)^{2}\right]=-2 \sinh v \sinh v^{\prime} \operatorname{Im} \zeta\right)$.

We can therefore rewrite the integral (54) as follows :

$$
\begin{aligned}
+i \int \omega\left(\vec{\alpha}^{\prime}\right) \int_{0}^{v}\left(\sinh v^{\prime}\right)^{d-2} \mathrm{~d} v^{\prime} & \\
& \int_{0 \leq \varphi^{\prime} \leq \varphi_{1}^{\prime}\left(v, v^{\prime}\right)} \Delta_{+} \mathcal{K} \times \mathcal{F}_{+}\left(\sinh \varphi^{\prime}\right)^{d-3} \mathrm{~d} \varphi^{\prime} .
\end{aligned}
$$

Now we must consider the contribution coming from the upper broken line $[-2 \varepsilon ;-\varepsilon+i v ; 0]$ (see Figure 3.d)). By proceeding as before, we obtain similarly :

$$
\begin{aligned}
-i \int \omega\left(\vec{\alpha}^{\prime}\right) \int_{0}^{v}\left(\sinh v^{\prime}\right)^{d-2} \mathrm{~d} v^{\prime} & \\
& \int_{0 \leq \varphi^{\prime} \leq \varphi_{1}^{\prime}\left(v, v^{\prime}\right)} \Delta_{+} \mathcal{K} \times \mathcal{F}_{-}\left(\sinh \varphi^{\prime}\right)^{d-3} \mathrm{~d} \varphi^{\prime} .
\end{aligned}
$$

In fact, instead of the situation of Figure 4.e), 4.f), we now have in the $\zeta$-plane the situation represented by Figure 4.g), 4.h), which yields (in the limit $\varepsilon \rightarrow 0$ ) the contribution from the integral (41) to the last term of Eq. (52) expressed by formula (56) (the limit of the cycle $\mathbf{L}_{\theta v^{\prime}}^{+}-\mathbf{L}_{\theta v^{\prime}}^{-}$being the same as before, illustrated on Figs. 5, 6). Finally, in view of formulae (55) and (56), the corresponding contribution to the discontinuity $\Delta \mathcal{G}$ (given by Eq. (52)) is (in view of Eq. (33)) :

$$
\begin{aligned}
\int \omega\left(\vec{\alpha}^{\prime}\right) \int_{\mu_{2}}^{v-\mu_{1}} & \left(\sinh v^{\prime}\right)^{d-2} \mathrm{~d} v^{\prime} \\
& \int_{0 \leq \varphi^{\prime} \leq \varphi_{1}^{\prime}\left(v, v^{\prime}\right)} \Delta_{+} \mathcal{K} \times \Delta_{+} \mathcal{F}\left(\sinh \varphi^{\prime}\right)^{d-3} \mathrm{~d} \varphi^{\prime}
\end{aligned}
$$

since $\Delta_{+} \mathcal{F}=0$ for $v^{\prime}<\mu_{2}$.

Now we must consider the contribution coming from the lower broken lines, namely $[-2 \varepsilon,-\varepsilon-i v, 0]$ (see Figure 3.c)) and $[0, \varepsilon-i v, 2 \varepsilon]$ (see Figure 3.d)). The previous analysis in the $\zeta$-plane remains valid, the new situation being now symmetric of the previous one under the symmetry 
$\left(\theta^{\prime}, \zeta\right) \mapsto\left(-\theta^{\prime},-\zeta\right)$ or $\left(\right.$ since $\left.\zeta=\cosh \varphi^{\prime}\right),\left(\theta^{\prime}, \varphi^{\prime}\right) \mapsto\left(-\theta^{\prime}, i \pi-\varphi^{\prime}\right)$. So the range of the integral (57) is now replaced by the set

$$
\left\{\left(\theta^{\prime}, \varphi^{\prime}\right) ; \theta^{\prime}=-i v^{\prime}, \mu_{2} \leq v^{\prime} \leq v-\mu_{1}, \varphi^{\prime} \in\left[i \pi, i \pi-\varphi_{1}^{\prime}\left(v, v^{\prime}\right)\right]\right\} .
$$

Then by using the identification (implied by $(R))\left(\theta^{\prime}, \varphi^{\prime}\right)=\left(-\theta^{\prime}, \varphi^{\prime}-i \pi\right)$ we obtain the following expression for this new contribution to $\Delta_{+} \mathcal{G}$ :

$$
\begin{aligned}
-\int \omega\left(\vec{\alpha}^{\prime}\right) \int_{\mu_{2}}^{v-\mu_{1}} & \left(\sinh v^{\prime}\right)^{d-2} \mathrm{~d} v^{\prime} \\
& \int_{0}^{-\varphi_{1}^{\prime}\left(v, v^{\prime}\right)} \Delta_{+} \mathcal{K} \Delta_{+} \mathcal{F}\left(\sinh \varphi^{\prime}\right)^{d-3} \mathrm{~d} \varphi^{\prime}
\end{aligned}
$$

Adding up the contributions (57) and (58) to $\Delta_{+} \mathcal{G}$, we obtain the complete expression :

$$
\int \omega\left(\vec{\alpha}^{\prime}\right) \int_{\mu_{2}}^{v-\mu_{1}}\left(\sinh v^{\prime}\right)^{d-2} \mathrm{~d} v^{\prime} \int_{-\varphi_{1}^{\prime}\left(v, v^{\prime}\right)}^{\varphi_{1}^{\prime}\left(v, v^{\prime}\right)} \Delta_{+} \mathcal{K} \Delta_{+} \mathcal{F}\left(\sinh \varphi^{\prime}\right)^{d-3} \mathrm{~d} \varphi^{\prime}
$$

In the general case $d>3$, it follows from the identification $\left(\theta^{\prime}, \varphi^{\prime}, \vec{\alpha}^{\prime}\right) \equiv$ $\left(\theta^{\prime},-\varphi^{\prime},-\vec{\alpha}^{\prime}\right)$ contained in $(R)$ (see Eqs. (20)), that the integral (58) reproduces the integral (57) if $\int \omega\left(\vec{\alpha}^{\prime}\right)$ is understood as the integral on the full sphere $\mathbb{S}_{d-3}$. Since the relation $R$ must be taken into account, $\int \omega\left(\vec{\alpha}^{\prime}\right)$ has then to be understood as $\frac{1}{2} \int_{\mathbb{S}_{d-3}} \omega\left(\vec{\alpha}^{\prime}\right)$ in formula (59). In the case $d=3$, formula (59) holds with $\int \omega\left(\vec{\alpha}^{\prime}\right)$ put equal to 1 .

In view of Eq. (19), the integration range of the integral (59) represents a region $\mathcal{R}$ of the real hyperboloid $X$ (illustrated in Figure 7 by its projection onto the space of coordinates $\left.z^{\prime(0)},\left|\left[\vec{z}^{\prime}\right]\right|\right)$ which is contained in the compact set $\diamond\left(z(i v), z_{0}\right)$. This region, which is bounded by the sheets of hyperboloid $z^{\prime 2}=\mu_{2}^{2}$, with $z^{\prime(0)}>0$ (i.e. $\left.v^{\prime}=\mu_{2}\right)$ and $\left(z-z^{\prime}\right)^{2}=\mu_{1}^{2}$, with $z^{(0)}-z^{(0)}>0$ (i.e. $\left.\varphi^{\prime}=\varphi_{1}\left(v, v^{\prime}\right), \vec{\alpha}^{\prime} \in \mathbb{S}_{d-3}, v^{\prime} \leq v-\mu_{1}\right)$ corresponds in fact to the intersection of the subsets $X_{\mu_{2}}^{+}$and $\left\{z^{\prime} ;\left(z(i v), z^{\prime}\right) \in \Sigma_{\mu_{1}}^{+}\right\}$in which the supports of $\Delta_{+} \mathcal{F}$ and $\Delta_{+} \mathcal{K}$ are respectively contained.

Formula (59) therefore represents (in terms of the parametrization (П)) the composition product $\Delta_{+} \mathcal{K} \diamond \Delta_{+} \mathcal{F}$ and yields Eq. (36) for the case $z=z(i v)$. The support of the function $\Delta_{+} \mathcal{G}$ thus obtained, is then determined by the condition that the region $\mathcal{R}$ is non-empty, namely (as easily seen on Figure 7) by the condition $z=z(i v)$ with $v \geq \mu_{1}+\mu_{2}$, i.e. $z \in X_{\mu, 0}^{+}$(which in fact corresponds to the result of Proposition 4).

$$
\text { TOME } 120-1992-\mathrm{N}^{\circ} 2
$$




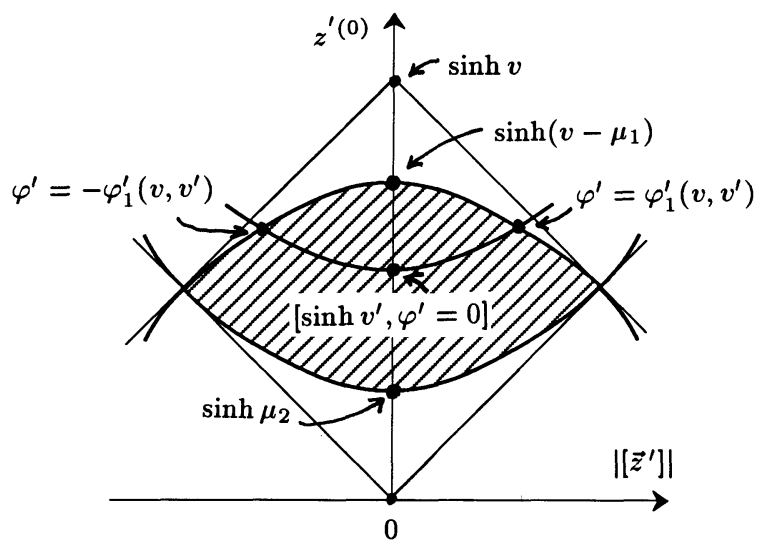

Figure 7. Hatchings represent the region $\mathcal{R}$.

We now come back to completing the proof of the fact that the function $\mathcal{G}(z(\theta))$ is analytic in the domain $\dot{\mathcal{J}}_{\mu}$. In view of the results of A), it remained to prove that $\mathcal{G}(z(\theta))$ is analytic on the sets $\{\theta=i v ;|v|<\mu\}$ and $\{\theta=\pi+i v ; v \in \mathbb{R}\}$.

Let us first consider the set $\{\theta=i v\}$. Since the previous computation of $\Delta_{+} \mathcal{G}(z(i v))$ is valid for all positive values of $v$, it follows from the support property of the integral (59) that $\Delta_{+} \mathcal{G}(z(i v))$ vanishes in the interval $0 \leq v<\mu$. Now, by using the symmetry property $D(\bar{\theta})=\overline{D(\theta)}$, we could give a similar computation for $\Delta_{-} \mathcal{G}(z(i v))$ in terms of $\Delta_{-} \mathcal{K}$ and $\Delta_{-} \mathcal{F}$ which would also imply that $\Delta_{-} \mathcal{G}(z(i v))$ vanishes for $-\mu<v \leq 0$. It follows that, for $z=z(i v)$, with $|v|<\mu$, the boundary values $\mathcal{G}_{+}$and $\mathcal{G}_{-}$ of $\mathcal{G}$ from both sides $\operatorname{Re} \theta>0$ and $\operatorname{Re} \theta<0$ coincide (although defined by different limiting cycles $\Gamma_{\theta}$ in the integral (41)); therefore, in view of the Schwarz-Painlevé [12] theorem (or "edge-of-the-wedge theorem" in one variable), these boundary values define the same function $\mathcal{G}(z(i v))$, analytic on the interval $|v|<\mu$.

We now consider the set $\{\theta=\pi+i v\}$. The boundary values of $\mathcal{G}(z(\theta))$ on this set from both sides $\operatorname{Re} \theta>\pi$ (or $\operatorname{Re} \theta>-\pi$ in view of the periodicity condition) and $\operatorname{Re} \theta<\pi$ are defined by taking limiting cycles $\Gamma_{\theta}$ in the integral (41), which are similar to those used above (for $\operatorname{Re} \theta$ tending to zero).

In fact (as a by-product of the analysis of 2) and 3) at the end of part A), making use of the symmetry properties $\left(S_{1}\right)$ ), the corresponding paths $\gamma_{\theta}$ contain broken lines neighbouring the line $\operatorname{Re} \theta=\pi$ which allow the same 
computation as above for the discontinuity function of $\mathcal{G}$ across the line $z=\pi+i v$. However, the resulting expression, which would be similar to the integral (59), would contain the discontinuity of $\mathcal{F}\left(z^{\prime}\left(\theta^{\prime}, \varphi^{\prime}, \vec{\alpha}^{\prime}\right)\right)$ across the set $\theta^{\prime}=\pi+i v^{\prime}$; but, since this set is contained in the analyticity domain $\mathcal{D}_{\mu_{2}}$ of $\mathcal{F}$, the discontinuity of $\mathcal{F}$ and therefore that of $\mathcal{G}$ vanish. By using as above the Schwarz-Painlevé theorem (now on the line $\theta=\pi+i v$, $v \in \mathbb{R}$ ), we conclude that $\mathcal{G}(z(\theta))$ is analytic on this line. This ends the proof of LEMMA 1.

\subsection{The algebra of perikernels.}

We shall now derive the following extension of THEOREM 1.

TheOREM $1^{\prime}$. - Let $\mathcal{K}_{1}, \mathcal{K}_{2}$ be perikernels in the respective classes $\mathcal{W}_{\mu_{1}}$ and $\mathcal{W}_{\mu_{2}}$. Then, there exists a unique perikernel $\mathcal{K}$ in the class $\mathcal{W}_{\mu}$, with $\mu=\mu_{1}+\mu_{2}$, denoted by $\mathcal{K}=\mathcal{K}_{1}{ }^{(c)} \mathcal{K}_{2}$, which satisfies the following properties

a)

$$
\mathcal{K}_{\mid S \times S}=\mathcal{K}_{1 \mid s \times S} * \mathcal{K}_{2 \mid s \times S}
$$

b)

$$
\Delta_{+} \mathcal{K}=\Delta_{+} \mathcal{K}_{1} \diamond \Delta_{+} \mathcal{K}_{2}
$$

c) For every $\left(z, z^{\prime}\right)$ in $\dot{\Delta}_{\mu}$, there exists a class of cycles $\dot{\Gamma}\left[z, z^{\prime}\right]$ in $H^{(d-1)}\left(D_{\mu_{1} \mu_{2}}^{z, z^{\prime}}\right)$, where :

$$
D_{\mu_{1} \mu_{2}}^{z, z^{\prime}}=\left\{z^{\prime \prime} \in X^{(c)} ;\left(z^{\prime \prime}, z^{\prime}\right) \in \dot{\Delta}_{\mu_{2}} ; \quad\left(z, z^{\prime \prime}\right) \in \dot{\Delta}_{\mu_{1}}\right\}
$$

such that:

$$
\mathcal{K}\left(z, z^{\prime}\right)=\int_{\dot{\Gamma}\left[z, z^{\prime}\right]} \mathcal{K}_{1}\left(z, z^{\prime \prime}\right) \mathcal{K}_{2}\left(z^{\prime \prime}, z^{\prime}\right) \mathrm{d} \sigma^{(c)}\left(z^{\prime \prime}\right)
$$

TheOrem $1^{\prime}$ can be presented as a corollary of TheOrem 1 , thanks to the following argument. We first prove property c) by determining for every $\left(z, z^{\prime}\right)$ in $\dot{\Delta}_{\mu}$ the corresponding class of cycles $\dot{\Gamma}\left[z, z^{\prime}\right]$ involved in Eq. (62). Since $z^{\prime}$ belongs to $\dot{X}^{(c)}$, we can apply Eq. (24) and write $z^{\prime}=z^{\prime}\left(\alpha^{\prime}, \varphi^{\prime}, \theta^{\prime}\right)=\mathbf{m}_{\alpha^{\prime} \varphi^{\prime} \theta^{\prime}} z_{0}$ with $\alpha^{\prime} \in \mathbb{S}_{d-3}^{(c)}, \varphi^{\prime} \in \mathbb{C}, \theta^{\prime} \in \mathbb{C}$; then we define the point $Z=\mathbf{m}_{\alpha^{\prime} \varphi^{\prime} \theta^{\prime}}^{-1} z$, which (as $z$ ) also belongs to $\dot{X}^{(c)}$ 
and moreover satisfies the condition $\left(Z-z_{0}\right)^{2}=\left(z-z^{\prime}\right)^{2} \neq \rho$, for all $\rho \geq \xi(\mu)$; this implies that $Z$ belongs to the domain $\dot{D}_{\mu}$ and that (in view of THEOREM 1) the corresponding class of cycles $\dot{\Gamma}(Z)$ is well-defined. We then introduce the cycle $\Gamma\left[z, z^{\prime}\right]$ by the following equation :

$$
\Gamma\left[z, z^{\prime}\right]=\mathbf{m}_{\alpha^{\prime} \varphi^{\prime} \theta^{\prime}} \Gamma(Z)
$$

where $\Gamma(Z)$ is the representative of $\dot{\Gamma}(Z)$ described in the proof of Theorem 1 (see Eq. (44)).

Since $\Gamma(Z)$ is contained in the domain

$$
D_{\mu_{1} \mu_{2}}^{Z}=\left\{Z^{\prime \prime} \in X^{(c)}, Z^{\prime \prime} \in \dot{D}_{\mu_{2}},\left(Z, Z^{\prime \prime}\right) \in \dot{\Delta}_{\mu_{1}}\right\},
$$

it follows that $\Gamma\left[z, z^{\prime}\right]$ is contained in the domain $D_{\mu_{1} \mu_{2}}^{z, z^{\prime}}$ and, therefore, that the integral $\int_{\Gamma\left[z, z^{\prime}\right]} \mathcal{K}_{1}\left(z, z^{\prime \prime}\right) \mathcal{K}_{2}\left(z^{\prime \prime}, z^{\prime}\right) \mathrm{d} \sigma^{(c)}\left(z^{\prime \prime}\right)$ defines a germ of analytic function at the given point $\left(z, z^{\prime}\right) \in \dot{\Delta}_{\mu}$. Since (in view of Eq. (63) and of the properties of $\Gamma(Z)) \Gamma\left[z, z^{\prime}\right]$ varies continuously with $\left(z, z^{\prime}\right)$ in the analyticity domain of $\mathcal{K}_{1}\left(z, z^{\prime \prime}\right) \mathcal{K}_{2}\left(z^{\prime \prime}, z^{\prime}\right)$ (in $\left(z, z^{\prime}, z^{\prime \prime}\right)$-complex space), it follows that the previous integral defines an analytic function $\mathcal{K}\left(z, z^{\prime}\right)$ in the domain $\dot{\Delta}_{\mu}$. We also see that the defining equation for $\mathcal{K}\left(z, z^{\prime}\right)$ can be rewritten as follows :

$$
\begin{aligned}
& \mathcal{K}\left(\mathbf{m}_{\alpha^{\prime} \varphi^{\prime} \theta^{\prime}} Z, \mathbf{m}_{\alpha^{\prime} \varphi^{\prime} \theta^{\prime}} z_{0}\right) \\
&=\int_{\Gamma(Z)} \mathcal{K}_{1}\left(\mathbf{m}_{\alpha^{\prime} \varphi^{\prime} \theta^{\prime}} Z, \mathbf{m}_{\alpha^{\prime} \varphi^{\prime} \theta^{\prime}} Z^{\prime \prime}\right) \\
& \quad \times \mathcal{K}_{2}\left(\mathbf{m}_{\alpha^{\prime} \varphi^{\prime} \theta^{\prime}} Z^{\prime \prime}, \mathbf{m}_{\alpha^{\prime} \varphi^{\prime} \theta^{\prime}} z_{0}\right) \mathrm{d} \sigma^{(c)}\left(Z^{\prime \prime}\right)
\end{aligned}
$$

(in view of Eq. (63) and of the invariance of the measure $\mathrm{d} \sigma^{(c)}$ under the transformation $\left.Z^{\prime \prime} \mapsto z^{\prime \prime}=\mathbf{m}_{\alpha^{\prime} \varphi^{\prime} \theta^{\prime}} Z^{\prime \prime}\right)$. By putting

$$
\begin{aligned}
\mathcal{F}\left(Z^{\prime \prime}\right) & =\mathcal{K}_{2}\left(\mathbf{m}_{\alpha^{\prime} \varphi^{\prime} \theta^{\prime}} Z^{\prime \prime}, \mathbf{m}_{\alpha^{\prime} \varphi^{\prime} \theta^{\prime}} z_{0}\right) \text { and } \\
\mathcal{G}(Z) & =\mathcal{K}\left(\mathbf{m}_{\alpha^{\prime} \varphi^{\prime} \theta^{\prime}} Z, \mathbf{m}_{\alpha^{\prime} \varphi^{\prime} \theta^{\prime}} z_{0}\right)
\end{aligned}
$$

we are led to the precise conditions for applying THEOREM 1, and we can draw the consequences of Eq. (35) and (36). Since Eq. (35) corresponds to putting $\Gamma(Z)=S, Z \in S$ and $\mathrm{d} \sigma^{(c)}=\mathrm{d} \boldsymbol{\sigma}$ in Eq. (64), (and since $\Gamma\left[z, z^{\prime}\right]=S$ in this case) we readily obtain property a) of the theorem (the operation $*$ being explicitly defined by Eq. (31)). Similarly in view of Eq. (36), we derive from Eq. (64) a formula for the discontinuity $\Delta \mathcal{K}$ 
in terms of $\Delta \mathcal{K}_{1}, \Delta \mathcal{K}_{2}$ which is exactly of the form of formula $\left(32^{\prime}\right)$ (equivalent to Eq. (32)) which yields the proof of property b) (Note that in view of Proposition 5, $\Delta \mathcal{K}$ thus belongs to the class $\left.W_{\mu_{1}+\mu_{2}}\right)$.

Finally, it remains to establish that the function $\mathcal{K}\left(z, z^{\prime}\right)$ defined for $\left(z, z^{\prime}\right)$ in $\dot{\Delta}_{\mu}$ by Eq. (62) admits an analytic continuation on the subset $\left\{\left(z, z^{\prime}\right) \in \Delta_{\mu} ; z \in \mathcal{C}_{ \pm}\right.$or $\left.z^{\prime} \in \mathcal{C}_{ \pm}\right\}$, where $\mathcal{C}_{+}$and $\mathcal{C}_{-}$are the $(d-2)$ dimensional cones defined in the proof of THEOREM 1 (before LEMma 1). We can follow closely the argument given there, since we now know that for each $z^{\prime}$ in $\dot{D}_{\mu}, \mathcal{K}\left(z, z^{\prime}\right)$ is analytic in a neighbourhood of the point $\left(z_{0}, z^{\prime}\right)$ in $X^{(c)} \times X^{(c)} \backslash \Sigma_{\mu}^{(c)}$. By then applying Bremermann's continuity theorem [11] in the variable $z$ (at $z^{\prime}$ fixed) as in the proof of THEOREM 1, we conclude that $\mathcal{K}$ can be analytically continued in the subset

$$
\left\{\left(z, z^{\prime}\right) ; z \in \mathcal{C}_{ \pm}, z^{\prime} \in \dot{D}_{\mu},\left(z, z^{\prime}\right) \notin \Sigma_{\mu}^{(c)}\right\} .
$$

By exchanging the roles of $z$ and $z^{\prime}$, one would similarly obtain the subset $\left\{\left(z, z^{\prime}\right) ; z \in \dot{D}_{\mu}, z^{\prime} \in \mathcal{C}_{ \pm},\left(z, z^{\prime}\right) \notin \Sigma_{\mu}^{(c)}\right\}$. The remaining points to be obtained in the analyticity domain of $\mathcal{K}$ are then :

$\alpha)$ Points $\left(z, z^{\prime}\right)$ such that $z \in \mathcal{C}_{ \pm}$(i.e. $\left.z^{(d-1)}= \pm 1\right)$ and $z^{\prime(d-1)}>$ $\cosh \mu$ (or $z^{(d-1)}>\cosh \mu$ and $z^{\prime} \in \mathcal{C}_{ \pm}$). Each of these points can be shown to belong to the holomorphy envelope of two polydisk-type regions contained in the previously obtained analyticity domain of $\mathcal{K}$ (see [13], [14] for such results based on a local application of the tube theorem).

$\beta)$ Points $\left(z, z^{\prime}\right)$ such that $z \in \mathcal{C}_{ \pm}$and $z^{\prime} \in \mathcal{C}_{ \pm}$. Since these points are contained in a complex analytic submanifold of codimension 2 surrounded by the domain previously obtained for the function $\mathcal{K}\left(z, z^{\prime}\right)$, the latter can be analytically continued at all these points.

\subsection{The case of distribution boundary values.}

Theorem 1 (resp. $1^{\prime}$ ) can be extended to the case where the function $\mathcal{F}$ and the perikernel $\mathcal{K}$ (resp. the two perikernels $\mathcal{K}_{1}, \mathcal{K}_{2}$ ) belong respectively to classes of the type $\mathcal{V}_{\mu_{2}, s_{2}}$ and $\mathcal{W}_{\mu_{1}, s_{1}}$ (resp. $\left.\mathcal{W}_{\mu_{1}, s_{1}}, \mathcal{W}_{\mu_{2}, s_{2}}\right)$ introduced in $\S 2.3$.

To be more specific, let us consider the definition of the function $\mathcal{G}(z)=\left(\mathcal{K}_{*}{ }^{(c)} \mathcal{F}\right)(z)$ of THEOREM 1 , in the special subset $D_{\mu, 0}$ of LEMMA 1. For every $z=z(\theta)$ in $D_{\mu, 0}$, the definition of $\mathcal{G}$ via Eq. (41) (with the cycle $\Gamma_{\theta}$ constructed in the proof of LEmma 1) remains valid, but one must now treat the problem of the boundary values of this integral, when the point $z$ tends to the boundary of $D_{\mu, 0}$, namely to the cut $\Xi_{\mu}$. In order to show that the boundary values $\mathcal{G}_{+}, \mathcal{G}_{-}$of $\mathcal{G}$ on $\Xi_{\mu}$ (and subsequently $\left.\Delta \mathcal{G}\right)$ 
exist in the sense of distributions, it is sufficient to prove that $|\mathcal{G}(z)|$ is bounded by an inverse power of the distance from $z$ to the set $\Xi_{\mu}$. This can be derived from the fact (implied by the assumptions $\mathcal{F} \in \mathcal{V}_{\mu_{2}, s_{2}}$ and $\mathcal{K} \in \mathcal{W}_{\mu_{1}, s_{1}}$ ) that $\left|\mathcal{F}\left(z^{\prime}\right)\right|$ (resp $\left|\mathcal{K}\left(z, z^{\prime}\right)\right|$ ) is itself bounded by an inverse power of the distance of $z^{\prime}$ from the set $\Xi_{\mu_{2}}$ (resp. of $\left(z, z^{\prime}\right)$ from the set $\left.\Sigma_{\mu_{1}}^{(c)}\right)$. To see this, one just has to notice that when $z=z(\theta)$ is at a distance $\varepsilon$ from $\Xi_{\mu}$ (i.e. when $\theta= \pm \varepsilon+i v$ ), the integration cycle $\Gamma_{\theta}$ remains at a distance bounded by Cst. $\times \varepsilon$ from the boundary of the domain of the integrand of Eq. (41); in fact, as shown respectively by Figure 3.c), 3.d) and Figure 4.e), 4.f), 4.g), 4.h), the cycle $\gamma_{\theta}$ remains at a distance $C_{1 \varepsilon}$ from the sets $\delta_{2}$ and $\delta_{1}$ in the $\theta^{\prime}$-plane and the cycle $\mathbf{L}_{\theta \theta^{\prime}}$ remains at a distance $C_{2 \varepsilon}$ from the set $\delta_{1}$ (a detailed computation which confirms this descriptive account can be given by using Eq. (45); in particular the angle of $\delta_{1}$ with the real $\zeta$-axis is of the order of $\varepsilon / \tanh v+\varepsilon^{\prime} / \tanh v^{\prime}$, if $\left.\theta=\varepsilon+i v, \theta^{\prime}=\varepsilon^{\prime}+i v^{\prime}\right)$.

By using this geometrical study, one can also check that (as in the case where $\mathcal{K}$ and $\mathcal{F}$ admit continuous boundary values), the integral $\mathcal{G}(z(\theta))$ is analytic at all points $\theta=i v$, with $|v|<\mu$.

It thus follows from this analysis that $\Delta \mathcal{G}(z(i v))=\left(i\left[\mathcal{G}_{+}-\mathcal{G}_{-}\right]\right)$ exists as a distribution whose support is contained in the set $X_{\mu, 0}^{+} \cup X_{\mu, 0}^{-}$ (i.e. $|v| \geq \mu$ ).

It remains to legitimate the formula $\Delta_{+} \mathcal{G}=\Delta_{+} \mathcal{K} \diamond \Delta_{+} \mathcal{F}$ (corresponding to Eq. (36)) in the present case where $\Delta_{+} \mathcal{G}, \Delta_{+} \mathcal{K}$ and $\Delta_{+} \mathcal{F}$ are distributions. On the one hand, we can consider this formula as a simple notation, which expresses the previous definiton of $\Delta_{+} \mathcal{G}$ through the construction of the analytic function $\mathcal{G}$. On the other hand, we can also try to justify this notation by a limiting procedure starting from situations where Eq. (27) is meaningful in the usual sense.

To this purpose, we consider the function $\mathcal{F}\left(z^{\prime}\left(\theta^{\prime}, \varphi^{\prime}, \vec{\alpha}^{\prime}\right)\right)$ and the function $\mathcal{K}\left(z(\theta), z^{\prime}\left(\theta^{\prime}, \varphi^{\prime}, \vec{\alpha}^{\prime}\right)\right)$ as limits of regularizing families of functions :

$$
\begin{aligned}
\mathcal{F}_{\eta}\left(z^{\prime}\left(\theta^{\prime}, \varphi^{\prime}, \alpha^{\prime}\right)\right) & =\int \mathcal{F}\left(z^{\prime}\left(\theta^{\prime}-i \hat{v}^{\prime}, \varphi^{\prime}, \vec{\alpha}^{\prime}\right)\right) \psi_{\eta}\left(\hat{v}^{\prime}\right) \mathrm{d} \hat{v}^{\prime} \text { and } \\
\mathcal{K}_{\eta}\left(z(\theta), z^{\prime}\left(\theta^{\prime}, \varphi^{\prime}, \vec{\alpha}^{\prime}\right)\right) & =\int \mathcal{K}\left(z(\theta-i \hat{v}), z^{\prime}\left(\theta^{\prime}, \varphi^{\prime}, \vec{\alpha}^{\prime}\right)\right) \psi_{\eta}(\hat{v}) \mathrm{d} \hat{v},
\end{aligned}
$$

where $\left\{\psi_{\eta}(\hat{v}) ; \eta>0, \eta \rightarrow 0\right\}$ denotes a family of $C^{\infty}$-functions with support contained in the interval $[-\eta,+\eta]$ and tending (weakly) to the Dirac measure $\delta$.

By using the definitions of the singular sets $\delta_{2}$ and $\delta_{1}$ of $\mathcal{F}$ and $\mathcal{K}$ (see in particular Eq. (45)), one can easily control (e.g. with the help of Figs. 3 
and 4) the small perturbations on these sets caused by the smearing out with the test-function $\psi_{\eta}$. One then notices that the integration contour $\Gamma_{\theta}$ (for $\theta= \pm \varepsilon+i v$ ) can still be chosen as in the proof of Lemma 1, so that the integral (41), with $\mathcal{K}$ and $\mathcal{F}$ now replaced by $\mathcal{K}_{\eta}$ and $\mathcal{F}_{\eta}$ still defines an analytic function $\mathcal{G}_{\eta}(z(\theta))$, whose boundary values $\left(\mathcal{G}_{\eta}\right)_{+}$and $\left(\mathcal{G}_{\eta}\right)_{-}$ on the line $\theta=i v$ are continuous.

The computation of the corresponding discontinuity function $\Delta_{+} \mathcal{G}_{\eta}$ can then be carried out along the same line as in part B) of the proof of LEMma 1 (since in the limit $\varepsilon \rightarrow 0$, the situation pictured in Fig. 5 remains valid, $\delta_{1}$ being along the real $\zeta$-axis, for all $\hat{v}$, with $|\hat{v}|<\eta$ ).

One therefore obtains for $\Delta_{+} \mathcal{G}_{\eta}$ an expression similar to that of formula (36), namely :

$$
\Delta_{+} \mathcal{G}_{\eta}(z(\theta))=\left(\Delta_{+} \mathcal{K}_{\eta} \diamond \Delta_{+} \mathcal{F}_{\eta}\right)(z(\theta))
$$

Then, since the analytic function $\mathcal{G}$ is obviously equal to the limit of the family $\mathcal{G}_{\eta}$ when $\eta$ tends to zero, it follows that $\Delta_{+} \mathcal{G}_{\eta}$ tends (weakly) to the distribution $\Delta_{+} \mathcal{G}$, so that we can write :

$$
\Delta_{+} \mathcal{G}=\lim _{\eta \rightarrow 0}\left(\Delta_{+} \mathcal{K}_{\eta} \diamond \Delta_{+} \mathcal{F}_{\eta}\right)
$$

which fully justifies the notation $\Delta_{+} \mathcal{K} \diamond \Delta_{+} \mathcal{F}$ for the r.h.s. of Eq. (65).

The previous analysis, which we gave for simplicity in the geometrical situation of lemma 1 , can be extended to the more general situations of TheOREM 1 and TheOREM $1^{\prime}$ by using the same matrix transformations as in the case of continuous boundary values.

\section{The microlocal version of Theorem 1.}

For every $\mathcal{F} \in \mathcal{V}_{\mu, s}$ (and for any order $s$ ), the corresponding distribution $\Delta_{+} \mathcal{F}$ has its support contained in $X_{\mu}^{+}$and is moreover analyticallydependent on the variables $(\varphi, \vec{\alpha})$ via the parametrization $z=z(\theta, \varphi, \vec{\alpha})$. Let us now consider $\Delta_{+} \mathcal{F}$ as a distribution on the space $\mathbb{R}^{d-1}$ of the variables $z^{0},[\vec{z}]=\left(z^{(1)}, \ldots, z^{(d-2)}\right)$, whose support is contained in the (one-to-one) projection $\mathbf{X}_{\mu}^{+}$of $X_{\mu}^{+}$, namely

$$
\mathbf{X}_{\mu}^{+}=\left\{\mathbf{z}=\left(z^{(0)},[\vec{z}]\right) ; \mathbf{z}^{2} \equiv z^{(0)^{2}}-[\vec{z}]^{2}=(\cosh \rho)^{2}, \rho \geq \mu\right\}
$$

In these variables, the distribution $\Delta_{+} \mathcal{F}$ thus considered is analytic along the sheets $\mathbf{z}^{2}=(\cosh \rho)^{2}$. According to the theory of "singular spectra" [6]

TOME $120-1992-\mathrm{N}^{\circ} 2$ 
(or "essential supports" [5]) of distributions, one can assert that its singular spectrum $S S\left(\Delta_{+} \mathcal{F}\right)$ is contained in the set

$$
N_{\mu}=\left\{(\mathbf{z}, \boldsymbol{\eta}) \in \mathbf{X}_{\mu}^{+} \times \mathbb{R}^{d-1} ; \boldsymbol{\eta}=\lambda \mathbf{z}, \lambda \in \mathbb{R}\right\}
$$

(provided we define the cotangent bundle to $\mathbb{R}^{d-1}$ via the minkowskian bilinear form : $\left.\boldsymbol{\xi} \cdot \boldsymbol{\eta}=\xi^{(0)} \eta^{(0}-\langle[\vec{\xi}],[\vec{\eta}]\rangle\right)$.

The microlocal extension of the previous class of distributions $\Delta_{+} \mathcal{F}$ is then characterized as the class $V_{\mu}^{(\text {loc })}$ of all distributions $F$ on $\mathbb{R}_{(\mathbf{z})}^{d-1}$, such that $\operatorname{supp} F \subset \mathbf{X}_{\mu}^{+}$and $S S(F) \subset N_{\mu}$.

Similarly, for every perikernel $\mathcal{K} \in \mathcal{W}_{\mu, s}$ (and for any order $s$ ), the corresponding distribution $\Delta_{+} \mathcal{K}$ (with $\operatorname{supp} \Delta_{+} \mathcal{K} \subset \Sigma_{\mu}^{+}$) can be considered as a distribution on the space $\mathbb{R}_{(\mathbf{z})}^{d-1} \times \mathbb{R}_{\left(\mathbf{z}^{\prime}\right)}^{d-1}$ whose support is contained in the projection $\Sigma_{\mu}^{+}$of $\Sigma_{\mu}^{+}$, namely

$$
\mathbf{\Sigma}_{\mu}^{+}=\left\{\left(\mathbf{z}, \mathbf{z}^{\prime}\right) ;\left(\mathbf{z}-\mathbf{z}^{\prime}\right)^{2}=(\cosh \rho)^{2}, \rho \geq \mu\right\}
$$

and which is moreover analytic along the sheets $\left(\mathbf{z}-\mathbf{z}^{\prime}\right)=(\cosh \rho)^{2}$. It follows that the singular spectrum $S S\left(\Delta_{+} \mathcal{K}\right)$ of $\Delta_{+} \mathcal{K}$ is contained in the set :

$$
\begin{aligned}
\Lambda_{\mu}=\left\{\left(\mathbf{z}, \mathbf{z}^{\prime}, \boldsymbol{\eta}, \boldsymbol{\eta}^{\prime}\right) \in \mathbf{\Sigma}_{\mu}^{+} \times\left(\mathbb{R}^{d-1} \times \mathbb{R}^{d-1}\right) ;\right. \\
\left.\boldsymbol{\eta}=\lambda\left(\mathbf{z}-\mathbf{z}^{\prime}\right), \boldsymbol{\eta}^{\prime}=-\lambda\left(\mathbf{z}-\mathbf{z}^{\prime}\right), \lambda \in \mathbb{R}\right\} .
\end{aligned}
$$

The microlocal extension of the previous class of distributions $\Delta_{+} \mathcal{K}$ is then characterized as the class $W_{\mu}^{(\text {loc })}$ of all distributions $K$ on $\mathbb{R}_{(\mathbf{z})}^{d-1} \times \mathbb{R}_{\left(\mathbf{z}^{\prime}\right)}^{d-1}$ such that $\operatorname{supp} K \subset \boldsymbol{\Sigma}_{\mu}^{+}$and $S S(K) \subset \Lambda_{\mu}$.

We can then state the following microlocal counterpart of THEOREM 1.

Proposition 6. - For every couple of distribution $(K, F)$ with $K$ in $W_{\mu_{1}}^{(\mathrm{loc})}$ and $F$ in $V_{\mu_{2}}^{(\mathrm{loc})}$, there exists a distribution $G=K \diamond F$, defined formally by Eq. (27), which belongs to the class $V_{\mu_{1}+\mu_{2}}^{(\mathrm{loc})}$ and satisfies the property $G=\lim _{\beta \rightarrow 0} K_{\beta} \diamond F_{\beta}$, for any regularizing families of functions $K_{\beta} \rightarrow K, F_{\beta} \rightarrow F$.

Proof. - We shall apply to Eq. (27) the theorems on products and integrals of distributions given in [5-b] (theorems 10 and 11). Let $\left(\mathbf{z}, \mathbf{z}^{\prime}\right)$ be any point in $\boldsymbol{\Sigma}_{\mu}^{+}$, such that $\mathbf{z}^{\prime} \in \mathbf{X}_{\mu_{2}}^{+}$. We then have :

$$
S S(K)_{\mid\left(\mathbf{z}, \mathbf{z}^{\prime}\right)} \subset\left\{\left(\boldsymbol{\eta}, \boldsymbol{\eta}^{\prime}\right) ; \boldsymbol{\eta}=-\boldsymbol{\eta}^{\prime}=\lambda_{1}\left(\mathbf{z}-\mathbf{z}^{\prime}\right)\right\},
$$


while $S S(F)_{\mid\left(\mathbf{z}, \mathbf{z}^{\prime}\right)} \subset\left\{\left(\boldsymbol{\eta}, \boldsymbol{\eta}^{\prime}\right) ; \boldsymbol{\eta}=0, \boldsymbol{\eta}^{\prime}=\lambda_{2} \mathbf{z}^{\prime}\right\}(F$ being considered as a distribution of $\left(\mathbf{z}, \mathbf{z}^{\prime}\right)$, constant with respect to $\left.\mathbf{z}\right)$. We first easily check that $S S(K)_{\mid\left(\mathbf{z}, \mathbf{z}^{\prime}\right)} \cap\left[-S S(F)_{\mid\left(\mathbf{z}, \mathbf{z}^{\prime}\right)}\right]=\phi$, which entails that the product $K\left(z, z^{\prime}\right) F\left(z^{\prime}\right)$ is well-defined and equal to the limit of $K_{\beta}\left(z, z^{\prime}\right) F_{\beta}\left(z^{\prime}\right)$ for any families of regularizing functions $K_{\beta}, F_{\beta}$. We now have :

$$
\begin{aligned}
& S S(K \cdot F)_{\mid\left(\mathbf{z}, \mathbf{z}^{\prime}\right)}=S S(K)_{\mid\left(\mathbf{z}, \mathbf{z}^{\prime}\right)}+\left.S S(F)\right|_{\left(\mathbf{z}, \mathbf{z}^{\prime}\right)} \\
& \subset\left\{\left(\boldsymbol{\eta}, \boldsymbol{\eta}^{\prime}\right) ; \boldsymbol{\eta}=\lambda_{1}\left(\mathbf{z}-\mathbf{z}^{\prime}\right),\right. \\
& \left.\boldsymbol{\eta}^{\prime}=-\lambda_{1} \mathbf{z}+\lambda_{1}^{\prime} \mathbf{z}^{\prime}, \lambda_{1} \in \mathbb{R}, \lambda_{1}^{\prime} \in \mathbb{R}\right\} .
\end{aligned}
$$

The integral (27), now written as

$$
G(\mathbf{z})=i(-1)^{d-1} \int_{\mathbf{z} \geq \mathbf{z}^{\prime} \geq 0} K\left(\mathbf{z}, \mathbf{z}^{\prime}\right) F\left(\mathbf{z}^{\prime}\right) \frac{\mathrm{d} z^{\prime 0} \wedge \cdots \wedge \mathrm{d} z^{\prime(d-2)}}{\sqrt{1+\mathbf{z}^{\prime 2}}}
$$

is then defined as a distribution (equal to $\lim _{\beta \rightarrow 0} \int K_{\beta} F_{\beta}$ ) whose singular spectrum satisfies the following formula:

$$
S S(G)_{\mid \mathbf{z}}=\bigcup_{\mathbf{z}^{\prime}} S S(K \cdot F)_{\mid\left(\mathbf{z}, \mathbf{z}^{\prime}\right), \eta^{\prime}=0}
$$

(with $\mathbf{z}^{\prime}$ varying in the integration domain).

In view of formula $\left(65^{\prime}\right)$, we must distinguish two cases :

i) if $\mathbf{z}^{\prime}$ is non-colinear to $\mathbf{z}, S S(K \cdot F)_{\mid\left(\mathbf{z}, \mathbf{z}^{\prime}\right), \boldsymbol{\eta}^{\prime}=0}$ reduces to $\boldsymbol{\eta}=0$.

ii) if $\mathbf{z}^{\prime}$ is colinear to $\mathbf{z}, S S(K \cdot F)_{\mid\left(\mathbf{z}, \mathbf{z}^{\prime}\right), \boldsymbol{\eta}^{\prime}=0}$ is contained in the fixed subset $\{\boldsymbol{\eta}=\lambda \mathbf{z} ; \lambda \in \mathbb{R}\}$.

It then follows that $S S(G)_{\mid \mathbf{z}} \subset\{\boldsymbol{\eta}=\lambda \mathbf{z} ; \lambda \in \mathbb{R}\}$. On the other hand, it still results from Proposition 4 that $\operatorname{supp} G \subset X_{\mu_{1}+\mu_{2}}^{+}$and therefore we conclude that $S S(G) \subset N_{\mu_{1}+\mu_{2}}$, i.e. that $G \subset V_{\mu_{1}+\mu_{2}}^{(\text {loc })}$.

\section{The algebra of crossing-symmetric perikernels on $X^{(c)}$}

In this last section, we shall consider classes of holomorphic functions $\mathcal{F}$ (resp. perikernels $\mathcal{K}$ ) defined in domains $\widehat{D}_{\mu}$ of $X^{(c)}$ (resp. in domains $\widehat{\Delta}_{\mu}$ of $X^{(c)} \times X^{(c)}$ ) bordered by two cuts, and we shall present properties of composition products for these classes which are the analogues of those stated in TheOREMs 1 and $1^{\prime}$.

TOME $120-1992-\mathrm{N}^{\circ} 2$ 


\subsection{Notations.}

i) Together with the subset $\Xi_{\mu}$ of $X^{(c)}$ defined in $\S 2.3$ i) (also described as $\left.\left\{z \in X^{(c)} ; z^{(d-1)} \geq \cosh \mu\right\}\right)$ we now introduce the subset :

$$
\begin{aligned}
\Xi_{\mu}^{\prime} & =\left\{z \in X^{(c)} ;\left(z+z_{0}\right)^{2}-\xi(\mu) \geq 0\right\} \\
& =\left\{z \in X^{(c)} ; z^{(d-1)} \leq-\cosh \mu\right\}
\end{aligned}
$$

and the "cut domain" $\widehat{D}_{\mu}=X^{(c)} \backslash\left(\Xi_{\mu} \cup \Xi_{\mu}^{\prime}\right)$ of $X^{(c)}$; we also put $\dot{\widehat{D}}_{\mu}=\dot{X}^{(c)} \backslash\left(\Xi_{\mu} \cup \Xi_{\mu}^{\prime}\right)$.

Similarly, together with the real subsets :

$$
X_{\mu}^{ \pm}=\Xi_{\mu} \cap X^{ \pm}=\left\{z \in X^{ \pm} ; z^{(d-1)} \geq \cosh \mu\right\} \subset Y,
$$

we introduce the corresponding subsets (see Fig. 8) :

$$
X_{\mu}^{\prime \pm}=\Xi_{\mu}^{\prime} \cap X^{\prime \pm}=\left\{z \in X^{\prime \pm} ; z^{(d-1)} \leq-\cosh \mu\right\} \subset Y^{\prime},
$$

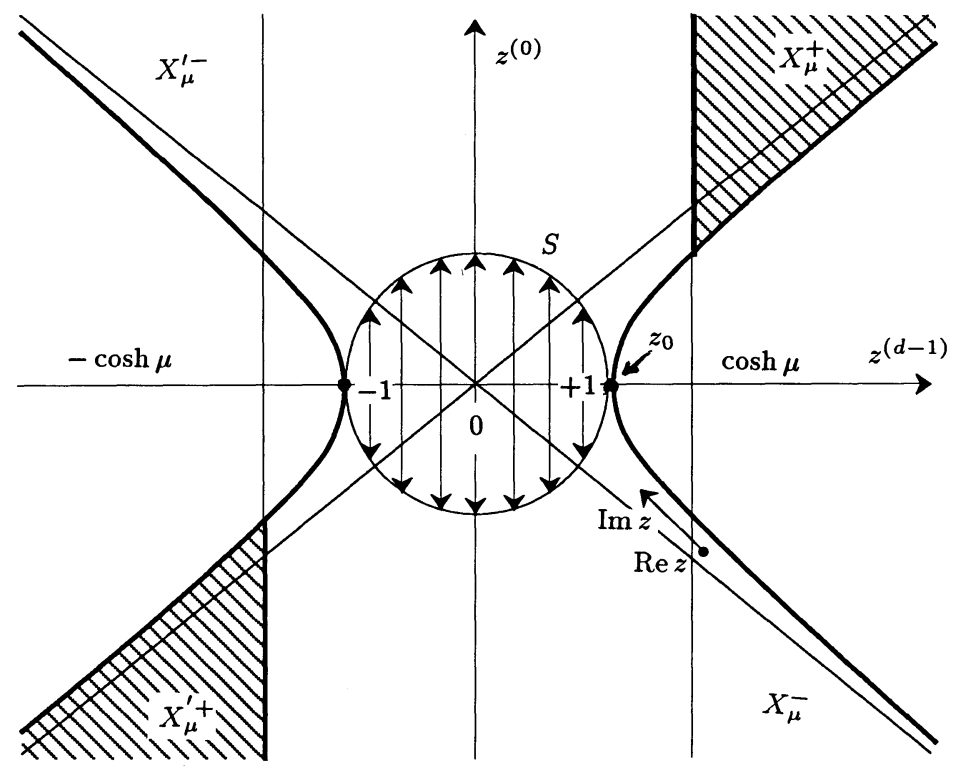

Figure 8. The hyperboloid $X$ and the regions $X_{\mu}^{+}, X_{\mu}^{\prime}$ (in hatchings) and $X_{\mu}^{-}, X_{\mu}^{\prime}$. A typical point $\operatorname{Re} z+i \operatorname{Im} z$ of $X^{(c)}$ and the sphere $S$ are illustrated via the "arrow representation" of complex points. 
where

$$
\begin{gathered}
X^{+}=\left\{z \in X ; z \geq z_{0}\right\}, \quad X^{-}=\left\{z \in X ; z \leq z_{0}\right\} \\
X^{\prime+}=\left\{z \in X ; z \leq-z_{0}\right\}, \quad X^{\prime-}=\left\{z \in X ; z \geq-z_{0}\right\} .
\end{gathered}
$$

Note that, according to the parametrization (I) (see Eq. (19)), the sets $X_{\mu}^{ \pm}$and $X_{\mu}^{\prime \pm}$ correspond respectively to the ranges $\{\theta=i v ; \pm v \geq \mu\}$ and $\{\theta=\pi+i v ; \pm v \geq \mu\}$, with $\varphi$ and $\vec{\alpha}$ real.

ii) Together with the subset $\Sigma_{\mu}^{(c)}$ of $X^{(c)} \times X^{(c)}$ defined in $\left.\S 2.3 \mathrm{i}^{\prime}\right)$, we now introduce the subset

$$
\Sigma_{\mu}^{(c)}=\left\{\left(z, z^{\prime}\right) \in X^{(c)} \times X^{(c)} ;\left(z+z^{\prime}\right)^{2}-\xi(\mu) \geq 0\right\}
$$

and the "cut domain" $\widehat{\Delta}_{\mu}=X^{(c)} \times X^{(c)} \backslash\left(\Sigma_{\mu}^{(c)} \cup \Sigma_{\mu}^{\prime(c)}\right)$ of $X^{(c)} \times X^{(c)}$. We also put $\dot{\widehat{\Delta}}_{\mu}=\dot{X}^{(c)} \times \dot{X}^{(c)} \backslash\left(\Sigma_{\mu}^{(c)} \cup \Sigma_{\mu}^{\prime(c)}\right)$.

Similarly, together with the real subsets $\Sigma_{\mu}^{ \pm}$(see Eq. (29)), we introduce the corresponding subsets :

$$
\Sigma_{\mu}^{\prime \pm}=\left\{\left(z, z^{\prime}\right) \in X \times X ;\left(z+z^{\prime}\right)^{2} \geq \xi(\mu), z^{(0)}+z^{(0)} \lessgtr 0\right\}
$$

and we note that for all real $\left(z, z^{\prime}\right)$ in $\Sigma_{\mu}^{+}$(resp. $\Sigma_{\mu}^{-}, \Sigma_{\mu}^{+}, \Sigma_{\mu}^{\prime-}$ ), the order relation $z-z^{\prime} \geq 0$ (resp. $z-z^{\prime} \leq 0, z+z^{\prime} \leq 0, z+z^{\prime} \geq 0$ ) is satisfied.

iii) We denote by $\hat{\mathcal{V}}_{\mu}$ the class of functions $\mathcal{F}(z)$ which are holomorphic in $\widehat{D}_{\mu}$ and have continuous boundary values on the set $\Xi_{\mu} \cup \Xi_{\mu}^{\prime}$ (from both sides of each of these cuts). For every function $\mathcal{F}$ in $\hat{\mathcal{V}}_{\mu}$, we put $\mathbf{F}=\mathcal{F}_{\mid \mathcal{S}}$ and we consider the associated "discontinuity functions" $\Delta \mathcal{F}=i\left(\mathcal{F}_{+}-\mathcal{F}_{-}\right)$and $\Delta^{\prime} \mathcal{F}=i\left(\mathcal{F}_{+}^{\prime}-\mathcal{F}_{-}^{\prime}\right)$ of $\mathcal{F}$, respectively across the analytic hypersurfaces

$$
\left\{z \in X^{(c)} ; \operatorname{Im}\left(z-z_{0}\right)^{2}=0\right\} \quad \text { and } \quad\left\{z \in X^{(c)} ; \operatorname{Im}\left(z+z_{0}\right)^{2}=0\right\} .
$$

The boundary values $\mathcal{F}_{+}, \mathcal{F}_{-}$of $\mathcal{F}$ are defined as in $\S 2.3$, and $\mathcal{F}_{+}^{\prime}, \mathcal{F}_{-}^{\prime}$ are defined similarly as boundary values of $\mathcal{F}$ (on the latter hypersurface) from the respective sides $\operatorname{Im}\left(z+z_{0}\right)^{2}<0$ and $\operatorname{Im}\left(z+z_{0}\right)^{2}>0$. Note that, together with formula (33) we also have :

$$
\mathcal{F}_{+}^{\prime}(z)=\lim _{\substack{\varepsilon \rightarrow 0 \\ \varepsilon>0}} \mathcal{F}[z(i v+\pi+\varepsilon, \varphi, \vec{\alpha})]
$$

TOME $120-1992-\mathrm{N}^{\circ} 2$ 
(since $\operatorname{Im}\left(z+z_{0}\right)^{2}=-2 \operatorname{Im} \cos (\pi+\varepsilon+i v)<0$ ).

The functions $\Delta \mathcal{F}$ and $\Delta^{\prime} \mathcal{F}$ have their supports respectively contained in the sets $\Xi_{\mu}$ and $\Xi_{\mu}^{\prime}$ and are analytic on all the corresponding submanifolds $\left(z-z_{0}\right)^{2}=\rho, \rho \geq \xi(\mu)$ and $\left(z+z_{0}\right)^{2}=\rho^{\prime}, \rho^{\prime} \geq \xi(\mu)$.

By taking the restrictions of these functions to the real hyperboloid $X$, we can give the following definitions : $\Delta_{ \pm} \mathcal{F}(z)=Y\left( \pm z^{(0)}\right) \Delta \mathcal{F}_{\mid X}(z)$, and $\Delta_{ \pm}^{\prime} \mathcal{F}(z)=Y\left(\mp z^{(0)}\right) \Delta^{\prime} \mathcal{F}_{\mid X}(z)$. The functions $\Delta_{+} \mathcal{F}, \Delta_{-} \mathcal{F}, \Delta_{+}^{\prime} \mathcal{F}, \Delta_{-}^{\prime} \mathcal{F}$ have their supports respectively contained in the sets $X_{\mu}^{+}, X_{\mu}^{-}, X_{\mu}^{\prime+}, X_{\mu}^{\prime-}$.

iv) We denote by $\widehat{\mathcal{W}}_{\mu}$ the class of functions $\mathcal{K}\left(z, z^{\prime}\right)$ (again called "perikernels") which are holomorphic in $\widehat{\Delta}_{\mu}$ and have continuous boundary values on the set $\Sigma_{\mu}^{(c)} \cup \Sigma_{\mu}^{\prime(c)}$ (from both sides of each of these cuts). For every perikernel $\mathcal{K}$ in $\widehat{\mathcal{W}}_{\mu}$, we put $\mathbf{K}=\mathcal{K}_{\mid \mathcal{S} \times \mathcal{S}}$ and we consider the associated "discontinuity functions" $\Delta \mathcal{K}=i\left(\mathcal{K}_{+}-\mathcal{K}_{-}\right)$and $\Delta^{\prime} \mathcal{K}=i\left(\mathcal{K}_{+}^{\prime}-\mathcal{K}_{-}^{\prime}\right)$ of $\mathcal{K}$ respectively across the analytic hypersurfaces

$$
\begin{array}{r}
\left\{\left(z, z^{\prime}\right) \in X^{(c)} \times X^{(c)} ; \operatorname{Im}\left(z-z^{\prime}\right)^{2}=0\right\} \quad \text { and } \\
\left\{\left(z, z^{\prime}\right) \in X^{(c)} \times X^{(c)} ; \operatorname{Im}\left(z+z^{\prime}\right)^{2}=0\right\} .
\end{array}
$$

The boundary values $\mathcal{K}_{+}, \mathcal{K}_{-}$of $\mathcal{K}$ are defined as in $\S 2.3$, and $\mathcal{K}_{+}^{\prime}, \mathcal{K}_{-}^{\prime}$ are defined similarly as boundary balues of $\mathcal{K}$ (on the latter hypersurface) from the respective sides $\operatorname{Im}\left(z+z^{\prime}\right)^{2}<0$ and $\operatorname{Im}\left(z+z^{\prime}\right)^{2}>0$. The functions $\Delta \mathcal{K}$ and $\Delta^{\prime} \mathcal{K}$ have their supports respectively contained in the sets $\Sigma_{\mu}^{(c)}$ and $\Sigma_{\mu}^{\prime(c)}$ and are analytic on all the corresponding submanifolds $\left(z-z^{\prime}\right)^{2}=\rho$, $\rho \geq \xi(\mu)$ and $\left(z+z^{\prime}\right)^{2}=\rho^{\prime}, \rho^{\prime} \geq \xi(\mu)$. By taking the restrictions of these functions to $X \times X$, we can give the following definitions :

$$
\begin{aligned}
& \Delta_{ \pm} \mathcal{K}\left(z, z^{\prime}\right)=\left.Y\left[ \pm\left(z^{(0)}-z^{(0)}\right)\right] \Delta \mathcal{K}\right|_{X \times X}\left(z, z^{\prime}\right), \\
& \Delta_{ \pm}^{\prime} \mathcal{K}\left(z, z^{\prime}\right)=\left.Y\left[\mp\left(z^{(0)}+z^{(0)}\right)\right] \Delta^{\prime} \mathcal{K}\right|_{X \times X}\left(z, z^{\prime}\right) .
\end{aligned}
$$

The kernels $\Delta_{+} \mathcal{K}, \Delta_{-} \mathcal{K}, \Delta_{+}^{\prime} \mathcal{K}, \Delta_{-}^{\prime} \mathcal{K}$ have their supports respectively contained in the sets $\Sigma_{\mu}^{+}, \Sigma_{\mu}^{-}, \Sigma_{\mu}^{\prime+}, \Sigma_{\mu}^{\prime-}$.

v) In $\S 2.2$, we defined the $\diamond$-composition product (see Eq. (32)) for kernels $K_{1}, K_{2}$ with supports contained in $\Sigma_{\mu_{1}}^{+}, \Sigma_{\mu_{2}}^{+}$(i.e. Volterra kernels). In the present case, we are led to extend this $\diamond$-product to classes of kernels with the more general support properties described above. One can easily see that eight possibilities emerge for defining a composition product by integration on a $\diamond$-like region; these possibilities correspond to the following order relations $\varepsilon z \geq z^{\prime \prime} \geq \varepsilon^{\prime} z^{\prime}$ and $\varepsilon^{\prime} z^{\prime} \geq z^{\prime \prime} \geq \varepsilon z$, 
with $\varepsilon, \varepsilon^{\prime}= \pm 1$, which express the various ways of combining the support properties " $\left(z, z^{\prime \prime}\right) \in \Sigma_{\mu_{1}}^{ \pm}$or $\Sigma_{\mu_{1}}^{ \pm \prime}$ and " $\left(z^{\prime \prime}, z^{\prime}\right) \in \Sigma_{\mu_{2}}^{ \pm}$or $\Sigma_{\mu_{2}}^{\prime \pm}$ ". In the following, the notation $K_{1} \diamond K_{2}$ will mean either

$$
\begin{aligned}
\int_{\diamond\left(\varepsilon z, \varepsilon^{\prime} z^{\prime}\right)} K_{1}\left(z, z^{\prime \prime}\right) K_{2}\left(z^{\prime \prime}, z^{\prime}\right) \mathrm{d} \sigma\left(z^{\prime \prime}\right) \quad \text { or } \\
\int_{\diamond\left(\varepsilon^{\prime} z^{\prime}, \varepsilon z\right)} K_{1}\left(z, z^{\prime \prime}\right) K_{2}\left(z^{\prime \prime}, z^{\prime}\right) \mathrm{d} \sigma\left(z^{\prime \prime}\right),
\end{aligned}
$$

the relevant choice of $\varepsilon, \varepsilon^{\prime}$ being given by the support properties of the kernels $K_{1}, K_{2}$ considered. Moreover, as in Proposition 5, each of the various kernels $K_{1} \diamond K_{2}$ thus obtained will have its support contained in one of the sets $\Sigma_{\mu_{1}+\mu_{2}}^{ \pm}$or $\Sigma_{\mu_{1}+\mu_{2}}^{ \pm}$.

All these considerations hold of course similarly for products of the form $K \diamond F$, if $F$ denotes any function whose support is contained in one of the sets $X_{\mu_{2}}^{ \pm}, X_{\mu_{2}}^{\prime \pm}$ (the variable $z^{\prime}$ being then replaced by the fixed point $z_{0}$ in all the previous formulæ).

\subsection{The composition product $*^{(c)}$.}

We can now state the following properties.

THEOREM 2. - Being given any kernel $\mathcal{K}$ in $\widehat{\mathcal{W}}_{\mu_{1}}$ and any function $\mathcal{F}$ in $\hat{\mathcal{V}}_{\mu_{2}}$, there exists a unique function $\mathcal{G}$ in the class $\hat{\mathcal{V}}_{\mu}$, with $\mu=\mu_{1}+\mu_{2}$, denoted by $\mathcal{K} *^{(c)} \mathcal{F}$ which satisfies the following properties:

a)

$$
\mathcal{G}_{\mid S}=\mathcal{K}_{\mid S \times S} * \mathcal{F}_{\mid S}
$$

b)

$$
\begin{aligned}
& \Delta_{+} \mathcal{G}=\Delta_{+} \mathcal{K} \diamond \Delta_{+} \mathcal{F}+\Delta_{-}^{\prime} \mathcal{K} \diamond \Delta_{+}^{\prime} \mathcal{F} ; \\
& \Delta_{+}^{\prime} \mathcal{G}=\Delta_{-} \mathcal{K} \diamond \Delta_{+}^{\prime} \mathcal{F}+\Delta_{+}^{\prime} \mathcal{K} \diamond \Delta_{+} \mathcal{F} .
\end{aligned}
$$

c) For every point $z$ in $\dot{\hat{D}}_{\mu}$, there exists a class of cycles $\dot{\widehat{\Gamma}}(z)$ in $H^{(d-1)}\left(\widehat{D}_{\mu_{1} \mu_{2}}^{z}\right)$, where $\widehat{D}_{\mu_{1} \mu_{2}}^{z}=\left\{z^{\prime} \in X^{(c)} ; z^{\prime} \in \widehat{D}_{\mu_{2}},\left(z, z^{\prime}\right) \in \dot{\widehat{\Delta}}_{\mu_{1}}\right\}$, such that:

$$
\mathcal{G}(z)=\int_{\grave{\widehat{\Gamma}}(z)} \mathcal{K}\left(z, z^{\prime}\right) \mathcal{F}\left(z^{\prime}\right) \mathrm{d} \sigma^{(c)}\left(z^{\prime}\right)
$$

TOME $120-1992-\mathrm{N}^{\circ} 2$ 
Theorem $2^{\prime}$. - Being given any couple of perikernels $\left(\mathcal{K}_{1}, \mathcal{K}_{2}\right)$ in the respective classes $\widehat{\mathcal{W}}_{\mu_{1}}, \widehat{\mathcal{W}}_{\mu_{2}}$, there exists a perikernel $\mathcal{K}$ in the class $\widehat{\mathcal{W}}_{\mu}$, with $\mu=\mu_{1}+\mu_{2}$, denoted by $\mathcal{K}=\mathcal{K}_{1} *^{(c)} \mathcal{K}_{2}$, which satisfies the following properties

a)

$$
\mathcal{K}_{\mid S \times S}=\mathcal{K}_{1 \mid S \times S} * \mathcal{K}_{2 \mid S \times S}
$$

b)

$$
\begin{aligned}
& \Delta_{+} \mathcal{K}=\Delta_{+} \mathcal{K}_{1} \diamond \Delta_{+} \mathcal{K}_{2}+\Delta_{-}^{\prime} \mathcal{K}_{2} \diamond \Delta_{+}^{\prime} \mathcal{K}_{2} ; \\
& \Delta_{+}^{\prime} \mathcal{K}=\Delta_{-} \mathcal{K}_{1} \diamond \Delta_{+}^{\prime} \mathcal{K}_{2}+\Delta_{+}^{\prime} \mathcal{K}_{1} \diamond \Delta_{+} \mathcal{K}_{2} .
\end{aligned}
$$

c) For every $\left(z, z^{\prime}\right)$ in $\dot{\widehat{\Delta}}_{\mu}$, there exists a class of cycles $\dot{\widehat{\Gamma}}\left[z, z^{\prime}\right]$ in $H^{(d-1)}\left(\widehat{D}_{\mu_{1} \mu_{2}}^{\left(z, z^{\prime}\right)}\right)$, where

$$
\widehat{D}_{\mu_{1} \mu_{2}}^{\left(z, z^{\prime}\right)}=\left\{z^{\prime \prime} \in X^{(c)} ;\left(z^{\prime \prime}, z^{\prime}\right) \in \dot{\widehat{\Delta}}_{\mu_{2}} ;\left(z, z^{\prime \prime}\right) \in \dot{\widehat{\Delta}}_{\mu_{1}}\right\}
$$

such that

$$
\mathcal{K}\left(z, z^{\prime}\right)=\int_{\hat{\widehat{\Gamma}}\left[z, z^{\prime}\right]} \mathcal{K}_{1}\left(z, z^{\prime \prime}\right) \mathcal{K}_{2}\left(z^{\prime \prime}, z^{\prime}\right) \mathrm{d} \sigma^{(c)}\left(z^{\prime \prime}\right) .
$$

Since the proofs of these theorems are very similar to those of Theorems 1 and $1^{\prime}$, we shall only sketch the proof of Theorem 2, Theorem $2^{\prime}$ being then derived from the latter in the same way as Theorem $1^{\prime}$ was derived from Theorem 1 .

As in the proof of THEOREM 1, we can reduce the general geometrical situation of Eq. (70) to that of Lemma 1, in which $z=z(\theta)=$ $(-i \sin \theta, \overrightarrow{0}, \cos \theta)$, and we are thus led to construct an appropriate $(d-1)-$ cycle $\hat{\Gamma}_{\theta}$ (in place of $\Gamma_{\theta}$ ) for the integral (41).

The cycle $\hat{\Gamma}_{\theta}$ is now submitted to the condition that its support is contained in the following domain $\widehat{D}(\theta)$ (instead of $D(\theta)$ ) :

$$
\begin{array}{r}
\widehat{D}(\theta)=\left\{\left(\theta^{\prime}, \varphi^{\prime}\right) \in \mathbb{C}^{2} ;\left(\theta^{\prime}, \varphi^{\prime}\right) \notin \Xi\left(\mu_{2}\right) \cup \Xi^{\prime}\left(\mu_{2}\right) \cup \Sigma\left(\mu_{1} ; \theta\right) \cup \Sigma^{\prime}\left(\mu_{1} ; \theta\right)\right\} \\
\times \mathbb{S}_{d-3}^{(c)} / R,
\end{array}
$$

BULLETIN DE LA SOCIÉTÉ MATHÉMATIQUE DE FRANCE 
with $\Xi\left(\mu_{2}\right)$ and $\Sigma\left(\mu_{1}, \theta\right)$ defined by Eqs. (39), (40) and

$$
\begin{aligned}
& \Xi^{\prime}\left(\mu_{2}\right)=\left\{\left(\theta^{\prime}, \varphi^{\prime}\right) \in \mathbb{C}^{2} ;\right. \\
& \left.\theta^{\prime}=i v^{\prime}+(2 k+1) \pi,\left|v^{\prime}\right| \geq \mu_{2}, k \in \mathbb{Z}\right\}
\end{aligned}
$$

(76) $\Sigma^{\prime}\left(\mu_{1} ; \theta\right)=\left\{\left(\theta^{\prime}, \varphi^{\prime}\right) \in \mathbb{C}^{2} ; \cos \theta \cos \theta^{\prime}+\sin \theta \sin \theta^{\prime} \cosh \varphi^{\prime}\right.$

$$
\left.\leq-\cosh \mu_{1}=-\rho_{1}\right\} \text {. }
$$

The cycle $\hat{\Gamma}_{\theta}$ will again be taken of the form

$$
\left\{\left(\theta^{\prime}, \varphi^{\prime}\right) ; \theta^{\prime} \in \hat{\gamma}_{\theta}, \varphi^{\prime} \in \hat{L}_{\theta \theta^{\prime}}\right\},
$$

but in order to satisfy the previous condition, it is required that :

a) $\hat{\gamma}_{\theta}$ should avoid not only $\delta_{2}$ (as $\gamma_{\theta}$ ) but also the subset

$$
\delta_{2}^{\prime}=\left\{\theta^{\prime}=i v^{\prime}+(2 k+1) \pi,\left|v^{\prime}\right| \geq \mu_{2}, k \in \mathbb{Z}\right\} .
$$

b) The image $\hat{\mathbf{L}}_{\theta \theta^{\prime}}$ of $\hat{L}_{\theta \theta^{\prime}}$ in the $\zeta$-plane should avoid not only $\delta_{1}$ (see Eq. (45)) but also the set :

$$
\delta_{1}^{\prime}=\left\{z \in \mathbb{C} ; \zeta \sin \theta \sin \theta^{\prime}+\cos \theta \cos \theta^{\prime}-\rho, \rho \leq-\rho_{1}\right\},
$$

which for $\theta$ or $\theta^{\prime} \notin \pi \mathbb{Z}$ is a half-line with the same support $\delta_{\theta \theta^{\prime}}$ as $\delta_{1}$.

The initial situation of $\hat{\Gamma}_{\theta}$ (for $\theta$ real) can still be chosen to coincide with that of $\Gamma_{\theta}$ (namely to represent the sphere $S$ ) since $\delta_{1}^{\prime} \cup \delta_{1}$ is contained in $]-\infty,-1[\cup]+1,+\infty$ [ (or empty) for all $\theta$ and $\theta^{\prime}$ real; Eq. (67) is then fulfilled.

The distortion $\hat{\gamma}_{\theta}$ is now submitted to the condition that $\hat{\gamma}_{\theta}$ should avoid not only the subset $\delta_{2} \cup \delta_{1}$, (as $\gamma_{\theta}$ in section 3) but also the subset $\delta_{2}^{\prime} \cup \delta_{1}^{\prime}$, where

$$
\delta_{1}^{\prime}=\left\{\theta^{\prime}=\theta+\pi+i v^{\prime} \text { or } \theta^{\prime}=-\theta+\pi+i v^{\prime}, \text { with }\left|v^{\prime}\right| \geq \mu_{1}\right\}
$$

(the latter set corresponding to situations where $\delta_{1}^{\prime}$ contains the points $\zeta=+1$ or $\zeta=-1$ and therefore violates condition b) above).

A convenient choice consists in defining $\hat{\gamma}_{\theta}$ as the (periodic) broken line whose vertices are at the points $\theta^{\prime}=-\theta, \theta-\pi, \pi-\theta, \theta, 2 \pi-\theta, \theta+\pi$, etc. (see Figure 9.a)). For every $\theta, \hat{\gamma}_{\theta}$ is seen to contain the real points $\theta^{\prime}=0$, $\frac{1}{2} \pi, \pi, \frac{3}{2} \pi$ etc.. The continuous distortion of $\hat{\gamma}_{\theta}$ from its initial situation (i.e. for example $[-\alpha, 2 \pi-\alpha]$ ) by dilatations parallel to the $v^{\prime}$-axis and proportional to $v$ is similar to that of $\gamma_{\theta}$ in section 3 .

The following definition of the path $\hat{\mathbf{L}}_{\theta \theta^{\prime}}$ (for all $\theta^{\prime}$ in $\hat{\gamma}_{\theta}$ ) can be here again motivated by the auxiliary lemma given in the course of the proof of Lemma 1. $\hat{\mathbf{L}}_{\theta \theta^{\prime}}$ is kept fixed and equal to the segment $[-1,+1]$ (oriented from +1 to -1 ) when $\theta^{\prime}$ belongs to the linear segments of $\hat{\gamma}_{\theta}$ which do not contain the points $\frac{1}{2} \pi$ and $\frac{3}{2} \pi$. 


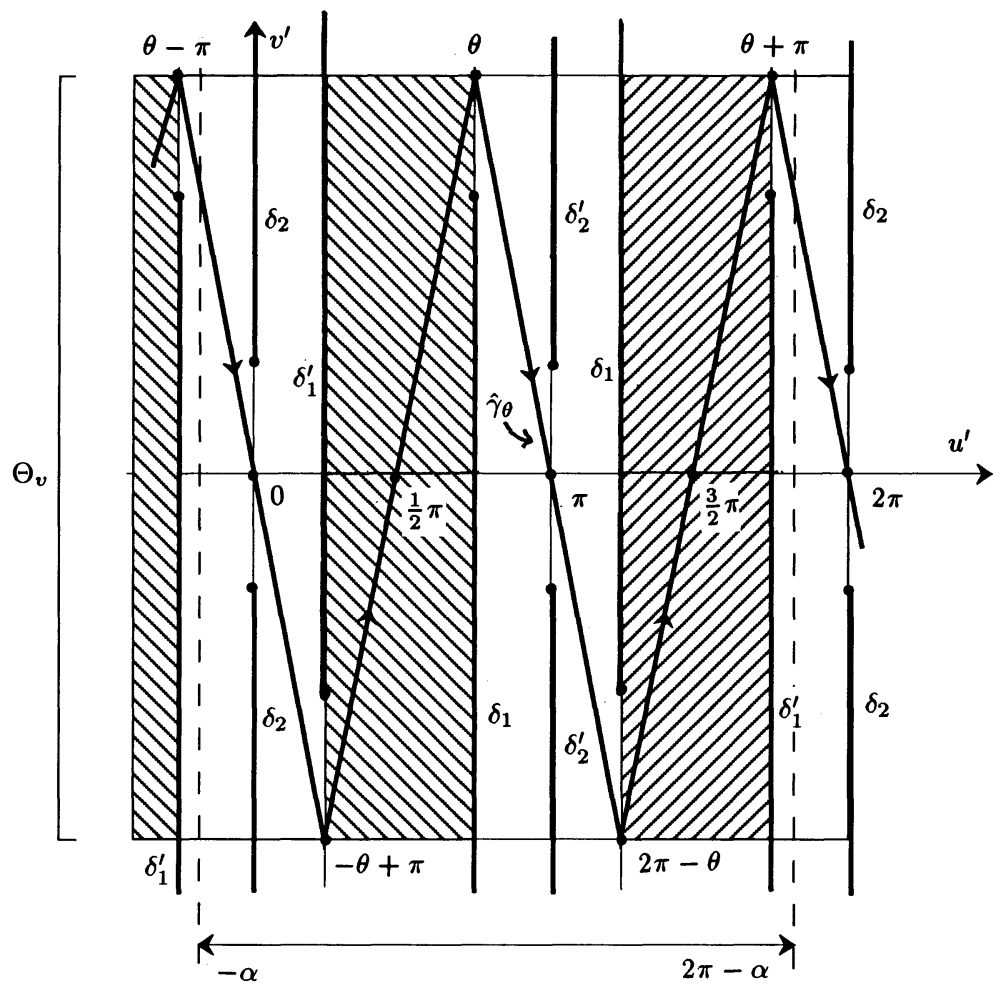

Figure 9.a : the general situation.

Figure 9 . The path $\hat{\gamma}_{\theta}$ in the $\theta^{\prime}$-plane.

When $\theta^{\prime}$ belongs to the remaining linear segments of $\hat{\gamma}_{\theta}$ (containing the points $\left.\frac{1}{2} \pi, \frac{3}{2} \pi\right), \hat{\mathbf{L}}_{\theta \theta^{\prime}}$ must be continuously distorted; a convenient choice consists in defining $\hat{\mathbf{L}}_{\theta \theta^{\prime}}$ as the broken line $\left[-1, \hat{\ell}_{\theta \theta^{\prime}}\right] \cup\left[\hat{\ell}_{\theta \theta^{\prime}}, 1\right]$ (see Fig. 10) oriented from +1 to -1 , with for instance (for $\theta^{\prime}$ in $[\theta, \pi-\theta]$ ),

$$
\begin{aligned}
\ell_{\theta \theta^{\prime}} & =\frac{-\cos \theta \cos \theta^{\prime}+\rho_{\theta}\left(\theta^{\prime}\right)}{\sin \theta \sin \theta^{\prime}} \text { and } \\
\rho_{\theta}\left(\theta^{\prime}\right) & =-\frac{\left|\theta-\theta^{\prime}\right|}{|2 \theta-\pi|}+\frac{\left|\pi-\theta-\theta^{\prime}\right|}{|2 \theta-\pi|}
\end{aligned}
$$

(since $\rho_{\theta}(\theta)=1$ and $\rho_{\theta}(\pi-\theta)=-1$, and therefore $\ell_{\theta \theta}=1$ and $\left.\ell_{\theta(\pi-\theta)}=-1\right)$. 


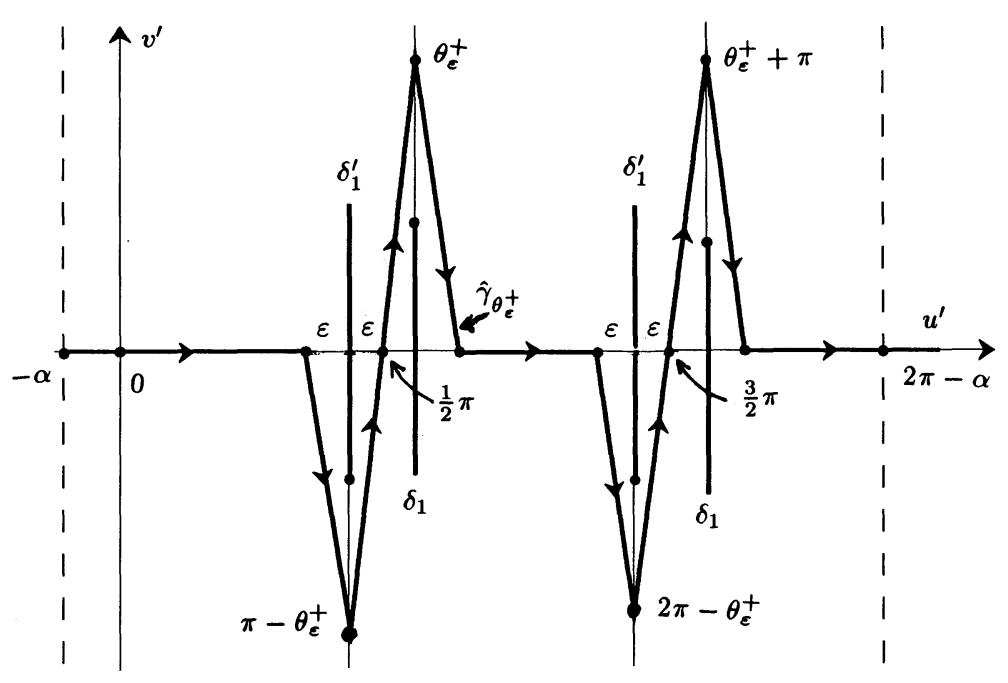

Figure 9.b : $\theta=\theta_{\varepsilon}^{+}$.

Figure 9. The path $\hat{\gamma}_{\theta}$ in the $\theta^{\prime}$-plane.

The previous construction of $\hat{\Gamma}_{\theta}$ holds for every $\theta=u+i v$ such that $u \neq 0, \frac{1}{2} \pi, \pi, \frac{3}{2} \pi$. The integral (70), written in the form (41) with $z=z(\theta)$ and $\hat{\Gamma}(z)=\hat{\Gamma}_{\theta}$, therefore defines an analytic function $\mathcal{G}(z(\theta))$ in the following subset of the $\theta$-plane :

$$
\widehat{D}_{0,0}=\left\{\theta=u+i v ; u \neq \frac{1}{2} k \pi, k \in \mathbb{Z}\right\} \cup \mathbb{R} / 2 \pi \mathbb{Z} .
$$

It remains to show that $\mathcal{G}(z(\theta))$ can be analytically continued on the lines $u=\frac{1}{2} \pi$ and $u=\frac{3}{2} \pi$ and to compute the discontinuity functions of $\mathcal{G}(z(\theta))$ across the lines $u=0$ and $u=\pi$, which will yield respectively formula (68) and (69) (for $z=z(\theta)$ ).

Let us first show that for $u=\frac{1}{2} \pi$, the limits of the cycle $\hat{\Gamma}_{\theta}$, although different from below and from above $\frac{1}{2} \pi$, yield the same value for the integral $\mathcal{G}\left(z\left(\frac{1}{2} \pi+i v\right)\right.$ ) so that (in view of the Schwarz-Painlevé theorem [12]) this common limit will be analytic on the line $u=\frac{1}{2} \pi$ (the case of the line $u=\frac{3}{2} \pi$ would be treated similarly).

Let $\mathcal{G}_{+}\left(z\left(\theta_{0}\right)\right)=\lim _{\substack{\varepsilon \rightarrow 0 \\ \varepsilon>0}} \mathcal{G}\left(z\left(\theta_{\varepsilon}^{+}\right)\right)$and $\mathcal{G}_{-}\left(z\left(\theta_{0}\right)\right)=\lim _{\substack{\varepsilon>0 \\ \varepsilon>0}} \mathcal{G}\left(z\left(\theta_{\varepsilon}^{-}\right)\right)$, where $\theta_{0}=\frac{1}{2} \pi+i v, \theta_{\varepsilon}^{+}=\frac{1}{2} \pi+\varepsilon+i v$ and $\theta_{\varepsilon}^{-}=\frac{1}{2} \pi-\varepsilon+i v$. For $\theta=\theta_{\varepsilon}^{+}$ and $\theta=\theta_{\varepsilon}^{-}$, the contour $\hat{\gamma}_{\theta}$ of Fig. 9.a) can be replaced by the broken lines indicated respectively on Fig. 9.b) and Fig. 9.c). 


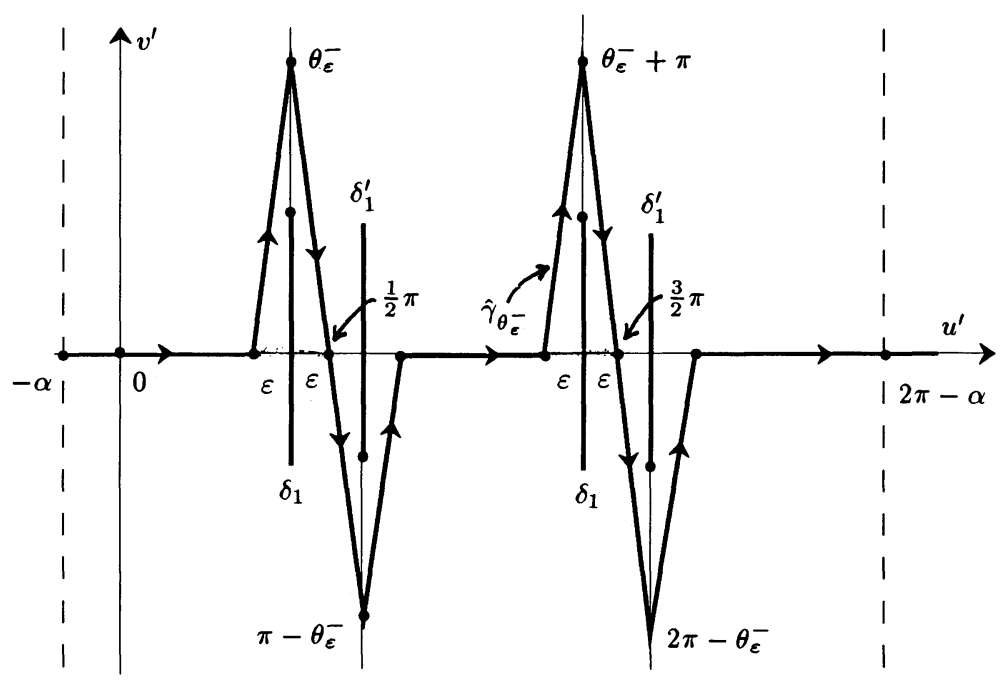

Figure 9.c : $\theta=\theta_{\varepsilon}^{-}$.

Figure 9. The path $\hat{\gamma}_{\theta}$ in the $\theta^{\prime}$-plane.

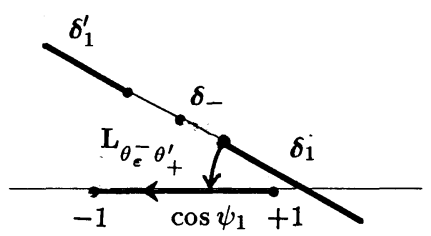

Figure 10.a $: \theta^{\prime}=\theta^{\prime+}$.

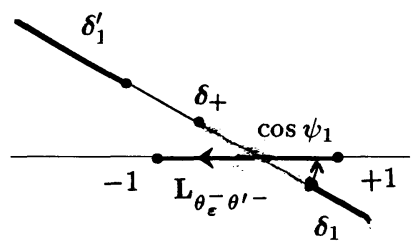

Figure 10.b : $\theta^{\prime}=\theta^{\prime-}$.

Figure 10. The path $\mathbf{L}_{\theta \theta^{\prime}}$ in the $\zeta$-plane for $\theta=\theta_{\varepsilon}^{-}$.

These pictures and the argument below correspond to the case when $v$ is positive; the case $v<0$ would be completely similar.

In view of the auxiliary lemma, the choice of $\mathbf{L}_{\theta \theta^{\prime}}$ equal to $[-1,+1]$ remains valid for all $\theta^{\prime}$ on the horizontal (real) parts of $\hat{\gamma}_{\theta_{\varepsilon}^{+}}$or $\hat{\gamma}_{\theta_{\varepsilon}^{-}}$and the corresponding contributions to the integral (70) written in the parametric form (41) have the same limits when $\theta_{\varepsilon}^{+}$and $\theta_{\varepsilon}^{-}$tend to the same point $\theta_{0}=\frac{1}{2} \pi+i v$.

We now have to compare (in this limiting situation) the contributions 
of the broken vertical parts of $\hat{\gamma}_{\theta}$ centered at $u^{\prime}=\frac{1}{2} \pi$ and $u^{\prime}=\frac{3}{2} \pi$. It is sufficient illustrative to consider the case when $\operatorname{Re} \theta^{\prime} \rightarrow \frac{1}{2} \pi$ and to compare the contributions (from $\theta_{\varepsilon}^{+}$and $\theta_{\varepsilon}^{-}$) of the upper parts of the broken line. In the $\zeta$-plane, the cuts $\boldsymbol{\delta}_{1}$ and $\boldsymbol{\delta}_{1}^{\prime}$ tend to a horizontal position when $\operatorname{Re} \theta$ and $\operatorname{Re} \theta^{\prime}$ tend to $\frac{1}{2} \pi$, since (in view of Eqs. (45) and (77)) their support is then described by the real equation

$$
\zeta=\frac{\sinh v \sinh v^{\prime}+\rho}{\cosh v \cosh v^{\prime}}
$$

For $\theta=\theta_{\varepsilon}^{-}, \boldsymbol{\delta}_{1}$ and $\boldsymbol{\delta}_{1}^{\prime}$ have their supporting line in a situation $\boldsymbol{\delta}_{-}$above the corresponding path $\mathbf{L}_{\theta_{\varepsilon}^{-} \theta^{\prime+}}=[-1,+1]$, when $\theta^{\prime+}=\theta^{-} \in\left[\frac{1}{2} \pi-2 \varepsilon, \theta_{\varepsilon}^{-}\right]$; this supporting line is in a situation $\boldsymbol{\delta}_{+}$which crosses the corresponding path $\mathbf{L}_{\theta_{\varepsilon}^{-} \theta^{\prime-}}$, when $\theta^{\prime}=\theta^{\prime-} \in\left[\theta_{\varepsilon}^{-}, \frac{1}{2} \pi\right]$, as illustrated on Fig. 10 .

By taking the limits $\theta_{\varepsilon}^{-} \rightarrow \theta_{0}$, one obtains the following contribution to $\mathcal{G}_{-}(z(\theta))$ coming from the upper broken lines (see Figure $9 \mathrm{c}$ )) :

$$
\begin{aligned}
& \int \omega\left(\vec{\alpha}^{\prime}\right) \int_{0}^{v-\mu_{1}}\left(\cosh v^{\prime}\right)^{d-2} \\
& \int_{0}^{\psi_{1}} \Delta \mathcal{K}\left(z\left(\theta_{0}\right), z^{\prime}\left(\frac{1}{2} \pi+i v^{\prime}, i \psi^{\prime}, \vec{\alpha}^{\prime}\right)\right) \\
& \mathcal{F}\left(z^{\prime}\left(\frac{1}{2} \pi+i v^{\prime}, i \psi^{\prime}, \vec{\alpha}^{\prime}\right)\right)\left(\sin \psi^{\prime}\right)^{d-3} \mathrm{~d} \psi^{\prime}
\end{aligned}
$$

where the limit $\psi_{1}$ corresponds to the origin of the cut $\delta_{1}$ (i.e. $\zeta_{1}=\cos \psi_{1}$ ) in $[-1,+1]$ (in view of Eq. (78),

$$
\left.\zeta_{1}=1-\frac{\cosh \left(v-v^{\prime}\right)-\cosh \mu_{1}}{\cosh v \cosh v^{\prime}}\right)
$$

and where $\Delta \mathcal{K}$ denotes the discontinuity of $\mathcal{K}$ across the set $\Sigma\left(\mu_{1} ; \theta\right)$.

By a similar analysis of the situation for $\theta=\theta_{\varepsilon}^{+}$, one would obtain, in the limit $\theta_{\varepsilon}^{+} \longrightarrow \theta_{0}$, the corresponding contribution to the expression of $\mathcal{G}_{+}$ coming from the upper broken lines near $\operatorname{Re} \theta^{\prime}=\frac{1}{2} \pi$ (see Fig. 9.b). One then easily checks that this contribution is identical with the integral (79). The analysis would be similar for the lower broken line contributions and also for the broken lines around $\operatorname{Re} \theta^{\prime}=\frac{3}{2} \pi$. It follows that $\mathcal{G}_{+}\left(z\left(\theta_{0}\right)\right)=$ $\mathcal{G}_{-}\left(z\left(\theta_{0}\right)\right)$ for all points $\theta_{0}=\frac{1}{2} \pi+i v$.

We shall not repeat the detailed analysis of the discontinuity of $\mathcal{G}(z(\theta))$ across $u=0$, since it is completely similar to the analysis given in the derivation of the discontinuity formula (36) of ThEOREM 1, except for 
the following point. For $\theta= \pm \varepsilon+i v$, the path $\gamma_{\theta}$ of Figs. 3.c), 3.d) must be replaced by a path $\hat{\gamma}_{\theta}$ which now exhibits two "folded vertical parts", namely a first one in the neighbourhood of the line $u^{\prime}=0$ (as for $\gamma_{\theta}$ ) and an additional one in the neighbourhood of the line $u^{\prime}=\pi$ (corresponding to a "pinching configuration" for the sets $\delta_{1}^{\prime}$ and $\delta_{2}^{\prime}$ ). While the first folded vertical part yields the first term at the r.h.s. of the discontinuity formula (68) (corresponding to the result of Eq. (36) in Theorem 1), the second folded vertical part gives rise to the last term of Eq. (68) (the exact form of the latter being obtained by a computation which is strictly similar). On the other hand, the analysis of $\mathcal{G}(z(\theta))$ for $\theta=\pi \pm \varepsilon+i v$ (which produced no discontinuity at $u=\pi$ in the case of THEOREM 1) now yields a situation in the $\theta^{\prime}$-plane involving again (as for $u=0$ ) two folded vertical parts of $\hat{\gamma}_{\theta}$ (corresponding respectively to "pinching configurations" for the sets $\left(\delta_{1}, \delta_{2}^{\prime}\right)$ and $\left(\delta_{1}^{\prime}, \delta_{2}\right)$ : these two parts respectively give rise to the two terms at the r.h.s. of Eq. (69).

Remark. - The extension of Theorems 2 and $2^{\prime}$ to the case when the functions $\mathcal{F}$ and perikernels $\mathcal{K}$ have boundary values in the sense of distributions, respectively on the sets $\Xi_{\mu}, \Xi_{\mu}^{\prime}$ and on the cuts $\Sigma_{\mu}^{(c)}, \Sigma_{\mu}^{\prime(c)}$, can be treated by the method described at the end of section 3 with similar results.

\section{BIBLIOGRAPHY}

[1-a] Faraut (J.). - Algèbre de Volterra et transformation de Laplace sphérique, Séminaire d'Analyse Harmonique de Tunis, 1981, exposé $\mathrm{n}^{\mathrm{o}} 29$.

[1-b] Faraut (J.). - Algèbres de Volterra et transformation de Laplace sphérique sur certains espaces symétriques ordonnés, Sympos. Math., t. 29, 1987 , p. 183-196.

[2] Faraut (J.) and Viano (G.A.). - Volterra algebra and the BetheSalpeter equation, J. Math. Phys., t. 27, 1986, p. 840-848.

[3] Hörmander (L.). - Uniqueness theorems and wavefront sets for solutions of linear differential equations with analytic coefficients, Comm. Pure Appl. Math., t. 24, 1971, p. 671-704. 
[4] Sato (M.), Kawai (T.) and Kashiwara (M.). - Hyperfunctions and pseudodifferential equations, Lecture Notes in Math., t. 287, 1973, p. 265-529.

[5-a] IAgolnitzer (D.). - Microlocal essential support of a distribution and decomposition theorems an introduction, in "Hyperfunctions and Theoretical Physics", Lecture Notes in Math., t. 449, 1975, p. $121-132$.

[5-b] Iagolnitzer (D.). - Analytic structure of distributions and essential support theory, in Structural analysis of collision amplitudes. R. Balian and D. Iagolnitzer, North Holland, Amsterdam, New York, Oxford, 1976.

[6] Bony (J.M.). - Équivalence des diverses notions de spectre singulier analytique, Séminaire Goulaouic-Schwartz, École Polytechnique, Palaiseau, 1976-77, exposé $\mathrm{n}^{\circ} 3$.

[7] Рнам (F.). - Introduction à l'étude topologique des singularités de Landau, Mémorial des Sciences Mathématiques 164. — GauthierVillars, Paris, 1967.

[8] Bros (J.) and Pesenti (D.). - Fredholm theory in complex manifolds with complex parameters : analyticity properties and Landau singularities of the resolvent, J. Math. Pures Appl., t. 58, 198o, p. 375-401.

[9] Martineau (A.). - Distributions et valeurs au bord des fonctions holomorphes, Cours international d'été sur la théorie des distributions, Lisbonne 1964.

[10] LerAy (J.). - Le calcul différentiel et intégral sur une variété complexe (Problème de Cauchy III),, Bull. Soc. Math. France, t. 87, 1959, p. 81-180.

[11] Bremermann (H.J.). - Thesis, Schrift. Math. Inst. Univ. Münster $5,1951$.

[12] Painlevé (P.). - Sur les lignes singulières des fonctions analytiques, Ann. Fac. Sci. Toulouse Math. 2, t. B27, 1887, p. 27-29 (article reproduit dans "Oeuvres de Paul Painlevé", tome II, p. 55-57, Éditions du C.N.R.S., Paris, 1974).

[13-a] Bros (J.) and Glaser (V.). - L'enveloppe d'holomorphie de l'union de deux polycercles, preprint CERN, (unpublished), 1961.

[13-b] Bros (J.). - Les problèmes de construction d'enveloppes d'holomorphie en théorie quantique des champs, Séminaire Lelong, Paris, exposé $\mathrm{n}^{\circ} 8,1962$.

TOME $120-1992-\mathrm{N}^{\circ} 2$ 
[13-c] Bros (J.). - Propriétés algébriques et analytiques de la fonction de $n$ points en théorie quantique des champs, Thèse Univ. Paris, 1970.

[14] Bros (J.), Epstein (H.), Glaser (V.) and Stora (R.). - Quelques aspects globaux des problèmes d'edge-of-the-wedge, in "Hyperfunctions and Theoretical Physics", Lecture Notes in Math., t. 449, 1975, p. $185-218$. 